\title{
A Measurement of Atmospheric Neutrino Oscillation Parameters by Super-Kamiokande I
}

Y.Ashie, ${ }^{1}$ J.Hosaka, ${ }^{1}$ K.Ishihara, ${ }^{1}$ Y.Itow,${ }^{1}$ J.Kameda,${ }^{1}$ Y.Koshio,${ }^{1}$ A.Minamino,,${ }^{1}$ C.Mitsuda, ${ }^{1}$ M.Miura, ${ }^{1}$ S.Moriyama, ${ }^{1}$ M.Nakahata, ${ }_{1}^{1}$ T.Namba, ${ }^{1}$ R.Nambu, ${ }^{1}$ Y.Obayashi, ${ }^{1}$ M.Shiozawa, ${ }^{1}$ Y.Suzuki, ${ }^{1}$ Y.Takeuchi, ${ }^{1}$ K.Taki, ${ }^{1}$ S.Yamada,,${ }^{1}$ M.Ishitsuka, ${ }^{2}$ T.Kajita, ${ }^{2}$ K.Kaneyuki, ${ }^{2}$ S.Nakayama, ${ }^{2}$ A.Okada, ${ }^{2}$ K.Okumura, ${ }^{2}$ C.Saji, ${ }^{2}$ Y.Takenaga,${ }^{2}$ S.T.Clark, ${ }^{3}$ S.Desai, ${ }^{3}$ E.Kearns,${ }^{3}$ S.Likhoded, ${ }^{3}$ J.L.Stone, ${ }^{3}$ L.R.Sulak, ${ }^{3}$ W.Wang, ${ }^{3}$ M.Goldhaber, ${ }^{4}$ D.Casper, ${ }^{5}$ J.P.Cravens, ${ }^{5}$ W.Gajewski, ${ }^{5}$ W.R.Kropp,${ }^{5}$ D.W.Liu, ${ }^{5}$ S.Mine, ${ }^{5}$ M.B.Smy, ${ }^{5}$ H.W.Sobel, ${ }^{5}$ C.W.Sterner, ${ }^{5}$ M.R.Vagins,,${ }^{5}$ K.S.Ganezer, ${ }^{6}$ J.Hill, ${ }^{6}$ W.E.Keig, ${ }^{6}$ J.S.Jang, ${ }^{7}$ J.Y.Kim, ${ }^{7}$ I.T.Lim, ${ }^{7}$ K.Scholberg, ${ }^{8}$ C.W.Walter, ${ }^{8}$ R.W.Ellsworth, ${ }^{9}$ S.Tasaka, ${ }^{10}$ G.Guillian, ${ }^{11}$ A.Kibayashi, ${ }^{11}$ J.G.Learned, ${ }^{11}$ S.Matsuno, ${ }^{11}$ D.Takemori, ${ }^{11}$ M.D.Messier, ${ }^{12}$ Y.Hayato, ${ }^{13}$ A.K.Ichikawa, ${ }^{13}$ T.Ishida, ${ }^{13}$ T.Ishii, ${ }^{13}$ T.Iwashita, ${ }^{13}$ T.Kobayashi, ${ }^{13}$ T.Maruyama, ${ }^{13}$, 团 K.Nakamura, ${ }^{13}$ K.Nitta, ${ }^{13}$ Y.Oyama, ${ }^{13}$ M.Sakuda, ${ }^{13}$, f Y.Totsuka, ${ }^{13}$ A.T.Suzuki, ${ }^{14}$ M.Hasegawa, ${ }^{15}$ K.Hayashi, ${ }^{15}$ I.Kato, ${ }^{15}$ H.Maesaka, ${ }^{15}$ T.Morita, ${ }^{15}$ T.Nakaya, ${ }^{15}$ K.Nishikawa, ${ }^{15}$ T.Sasaki, ${ }^{15}$ S.Ueda ${ }^{15}$ S.Yamamoto, ${ }^{15}$ T.J.Haines,${ }^{16,5}$ S.Dazeley,${ }^{17}$ S.Hatakeyama, ${ }^{17}$ R.Svoboda,,${ }^{17}$ E.Blaufuss, ${ }^{18}$ J.A.Goodman,,${ }^{18}$ G.W.Sullivan, ${ }^{18}$ D.Turcan, ${ }^{18}$ A.Habig, ${ }^{19}$ Y.Fukuda, ${ }^{20}$ C.K.Jung, ${ }^{21}$ T.Kato, ${ }^{21}$ K.Kobayashi, ${ }^{21}$ M.Malek, ${ }^{21}$ C.Mauger, ${ }^{21}$ C.McGrew, ${ }^{21}$ A.Sarrat,${ }^{21}$ E.Sharkey, ${ }^{21}$ C. Yanagisawa, ${ }^{21}$ T.Toshito, ${ }^{22}$ K.Miyano, ${ }^{23}$ N.Tamura, ${ }^{23}$ J.Ishii, ${ }^{24}$ Y.Kuno, ${ }^{24}$ M.Yoshida, ${ }^{24}$ S.B.Kim,,${ }^{25}$ J.Yoo, ${ }^{25}$ H.Okazawa, ${ }^{26}$ T.Ishizuka, ${ }^{27}$ Y.Choi, ${ }^{28}$ H.K.Seo, ${ }^{28}$ Y.Gando, ${ }^{29}$ T.Hasegawa, ${ }^{29}$ K.Inoue, ${ }^{29}$ J.Shirai, ${ }^{29}$ A.Suzuki, ${ }^{29}$ M.Koshiba, ${ }^{30}$ Y.Nakajima, ${ }^{31}$ K.Nishijima, ${ }^{31}$ T.Harada, ${ }^{32}$ H.Ishino, ${ }^{32}$ Y.Watanabe,${ }^{32}$ D.Kielczewska, ${ }^{33,5}$ J.Zalipska, ${ }^{33}$ H.G.Berns, ${ }^{34}$ R.Gran, ${ }^{34}$ K.K.Shiraishi, ${ }^{34}$ A.Stachyra, ${ }^{34}$ K.Washburn, ${ }^{34}$ and R.J.Wilkes ${ }^{34}$

(The Super-Kamiokande Collaboration)

\footnotetext{
${ }^{1}$ Kamioka Observatory, Institute for Cosmic Ray Research, University of Tokyo, Kamioka, Gifu, 506-1205, Japan

${ }^{2}$ Research Center for Cosmic Neutrinos, Institute for Cosmic Ray Research, University of Tokyo, Kashiwa, Chiba 277-8582, Japan

${ }^{3}$ Department of Physics, Boston University, Boston, MA 02215, USA

${ }^{4}$ Physics Department, Brookhaven National Laboratory, Upton, NY 11973, USA

${ }^{5}$ Department of Physics and Astronomy, University of California, Irvine, Irvine, CA 92697-4575, USA

${ }^{6}$ Department of Physics, California State University, Dominguez, Hills, Carson, CA 90747, USA

${ }^{7}$ Department of Physics, Chonnam National University, Kwangju 500-757, Korea

${ }^{8}$ Department of Physics, Duke University, Durham, NC 27708, USA

${ }^{9}$ Department of Physics, George Mason University, Fairfax, VA 22030, USA

${ }^{10}$ Department of Physics, Gifu University, Gifu, Gifu 501-1193, Japan

${ }^{11}$ Department of Physics and Astronomy, University of Hawaii, Honolulu, HI 96822, USA

${ }^{12}$ Department of Physics, Indiana University, Bloomington, IN 47405-7105, USA

${ }^{13}$ High Energy Accelerator Research Organization (KEK), Tsukuba, Ibaraki 305-0801, Japan

${ }^{14}$ Department of Physics, Kobe University, Kobe, Hyogo 657-8501, Japan

${ }^{15}$ Department of Physics, Kyoto University, Kyoto 606-8502, Japan

${ }^{16}$ Physics Division, P-23, Los Alamos National Laboratory, Los Alamos, NM 87544, USA

${ }^{17}$ Department of Physics and Astronomy, Louisiana State University, Baton Rouge, LA 70803, USA

${ }^{18}$ Department of Physics, University of Maryland, College Park, MD 20742, USA

${ }^{19}$ Department of Physics, University of Minnesota, Duluth, MN 55812-2496, USA

${ }^{20}$ Department of Physics, Miyagi University of Education, Sendai,Miyagi 980-0845, Japan

${ }^{21}$ Department of Physics and Astronomy, State University of New York, Stony Brook, NY 11794-3800, USA

${ }^{22}$ Department of Physics, Nagoya University, Nagoya, Aichi 464-8602, Japan

${ }^{23}$ Department of Physics, Niigata University, Niigata, Niigata 950-2181, Japan

${ }^{24}$ Department of Physics, Osaka University, Toyonaka, Osaka 560-0043, Japan

${ }^{25}$ Department of Physics, Seoul National University, Seoul 151-742, Korea

${ }^{26}$ International and Cultural Studies, Shizuoka Seika College, Yaizu, Shizuoka 425-8611, Japan

${ }^{27}$ Department of Systems Engineering, Shizuoka University, Hamamatsu, Shizuoka 432-8561, Japan

${ }^{28}$ Department of Physics, Sungkyunkwan University, Suwon 440-746, Korea

${ }^{29}$ Research Center for Neutrino Science, Tohoku University, Sendai, Miyagi 980-8578, Japan

${ }^{30}$ University of Tokyo, Tokyo 113-0033, Japan

${ }^{31}$ Department of Physics, Tokai University, Hiratsuka, Kanagawa 259-1292, Japan

${ }^{32}$ Department of Physics, Tokyo Institute for Technology, Meguro, Tokyo 152-8551, Japan

${ }^{33}$ Institute of Experimental Physics, Warsaw University, 00-681 Warsaw, Poland

${ }^{34}$ Department of Physics, University of Washington, Seattle, WA 98195-1560, USA
}

(Dated: February 3, 2008)

\begin{abstract}
We present a combined analysis of fully-contained, partially-contained and upward-going muon atmospheric neutrino data from a 1489 day exposure of the Super-Kamiokande detector. The data samples span roughly five decades in neutrino energy, from $100 \mathrm{MeV}$ to $10 \mathrm{TeV}$. A detailed Monte Carlo comparison is described and presented. The data is fit to the Monte Carlo expectation, and is found to be consistent with neutrino oscillations of $v_{\mu} \leftrightarrow v_{\tau}$ with $\sin ^{2} 2 \theta>0.92$ and $1.5 \times 10^{-3}<\Delta m^{2}<3.4 \times 10^{-3} \mathrm{eV}^{2}$ at $90 \%$ confidence level.
\end{abstract}


PACS numbers: PACS numbers: 14.60.Pq, 96.40.Tv

\section{INTRODUCTION}

Atmospheric neutrinos are produced from the decays of particles resulting from interactions of cosmic rays with Earth's atmosphere. We have previously reported the results of a number of atmospheric neutrino observations spanning energies from $100 \mathrm{MeV}$ to $10 \mathrm{TeV}$ [1, 2, 3, 4]. In each case, a significant zenith-angle dependent deficit of $v_{\mu}$ was observed. These deficits have been interpreted as evidence for neutrinos oscillations [5]. If neutrinos have a non-zero mass, then the probability that a neutrino of energy $E_{v}$ produced in a weak flavor eigenstate $v_{\alpha}$ will be observed in eigenstate $v_{\beta}$ after traveling a distance $L$ through the vacuum is:

$$
P\left(v_{\alpha} \rightarrow v_{\beta}\right)=\sin ^{2} 2 \theta \sin ^{2}\left(\frac{1.27 \Delta m^{2}\left(\mathrm{eV}^{2}\right) L(\mathrm{~km})}{E_{v}(\mathrm{GeV})}\right),
$$

where $\theta$ is the mixing angle between the mass eigenstates and the weak eigenstates and $\Delta m^{2}$ is the difference of the squared mass eigenvalues. This equation is valid in the 2flavor approximation. The analysis reported in this paper is under the assumption of effective 2-flavor neutrino oscillations, $v_{\mu} \leftrightarrow v_{\tau}$, which is considered to be dominant in atmospheric neutrino oscillations. Equation 1 is also true in matter for $v_{\mu} \leftrightarrow v_{\tau}$, but may be modified for oscillation involving $v_{e}$ which travel through matter. The zenith angle dependence of the observed deficits results from the variation of $L$ with the direction of the neutrino. Neutrinos produced directly overhead travel roughly $15 \mathrm{~km}$ to the detector while those produced directly below traverse the full diameter of the Earth $(13,000 \mathrm{~km})$ before reaching the detector. By measuring the neutrino event rate over these wide ranges of $E_{\mathrm{v}}$ and $L$, we have measured the neutrino oscillation parameters $\Delta m^{2}$ and $\sin ^{2} 2 \theta$.

Super-Kamiokande (also Super-K or SK) is a 50-kiloton water Cherenkov detector located deep underground in Gifu Prefecture, Japan. Atmospheric neutrinos are observed in Super-K in two ways. At the lowest energies, $100 \mathrm{MeV}-$ $10 \mathrm{GeV}$, atmospheric neutrinos are observed via their chargedcurrent interactions with nuclei in the 22.5 kiloton water fiducial mass: $v+N \rightarrow l+X$. These interactions are classified as fully-contained (FC) if all of the energy is deposited inside the inner Super-K detector, or as partially-contained (PC) if a high energy muon exits the inner detector, depositing energy in the outer veto region. The neutrino energies that produce partially-contained events are typically 10 times higher than those that produce fully-contained events. The Super-K detector started observation on April, 1996 achieving a 92 kilotonyr (1489 live-day) exposure to atmospheric neutrinos through July, 2001 during the Super-Kamiokande I running period.

\footnotetext{
*Present address: Department of Physics, Univ. of Tsukuba, Tsukuba, Ibaraki 3058577 , Japan

${ }^{\dagger}$ Present address: Department of Physics, Okayama University, Okayama
} 700-8530, Japan

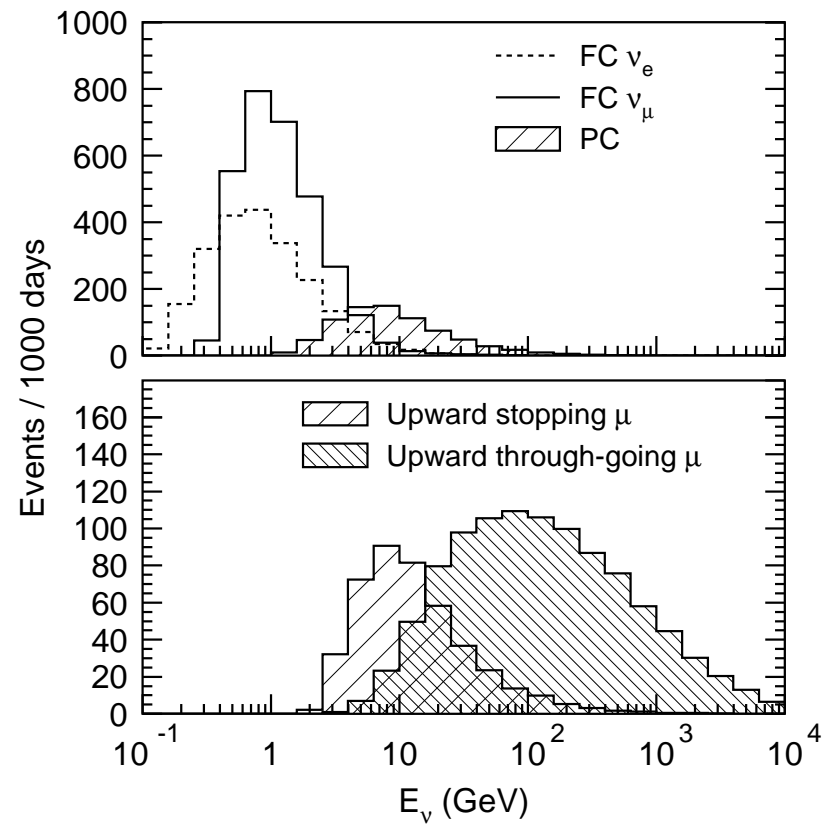

FIG. 1: The parent neutrino energy distributions for the fullycontained, partially-contained, upward stopping-muon and upward through-going muons samples. Rates for the fully-contained and partially-contained samples are for interactions in the 22.5 kiloton fiducial volume. Taken together, the samples span five decades in neutrino energy.

Neutrinos can also be detected by their interactions with the rock surrounding the detector. Charged-current $v_{\mu}$ interactions with the rock produce high energy muons which intersect the detector. While these interactions can not be distinguished from the constant rain of cosmic ray muons traveling in the downward direction, muons traveling in an upward direction through the detector must be neutrino induced. Upward-going muon events are separated into two categories: those that come to rest in the detector (upward stopping muons) and those that traverse the entire detector volume (upward through-going muons). The energies of the neutrinos which produce stopping muons are roughly the same as for partially-contained events, $\sim 10 \mathrm{GeV}$. Upward through-going events, however, are significantly more energetic; the parent neutrino energy for these events is about $100 \mathrm{GeV}$ on average.

Figure 1 shows the expected number of neutrino events in each event category as a function of neutrino energy. The samples taken together span nearly five decades in energy. This broad range of available energies, in combination with the variation in neutrino travel distance, makes the combined data sample well suited for a precise measurement of neutrino oscillation parameters.

There have been numerous other measurements of atmospheric neutrinos. Kamiokande [6, 7], IMB [8, 9] and Soudan $2[10,11]$ observed significantly smaller $v_{\mu}$ to $v_{e}$ flux ratios of $\sim 1 \mathrm{GeV}$ atmospheric neutrinos, which were interpreted as a signature for neutrino oscillation. The ratio was 
used in order to normalize the uncertainty in the overall atmospheric neutrino flux. Data on multi-GeV atmospheric neutrino events [12] and upward-going muons [13, 14, 15] have also shown a zenith-angle dependent deficit of the $v_{\mu}$ flux. The $v_{\mu} \leftrightarrow v_{\tau}$ oscillation analyses of these various data over various energy ranges $[3,4,5,12,14,15,16,17,18]$ indicated similar $\Delta m^{2}$ and $\sin ^{2} 2 \theta$ regions as the first measurements from Super-K as well as those reported here.

The K2K long baseline experiment used an accelerator beamline to produce muon neutrinos that traveled $250 \mathrm{~km}$ to the Super-K detector, as a means to study neutrino oscillation in the atmospheric neutrino energy and distance scales. The results from $\mathrm{K} 2 \mathrm{~K}$ [19,20] are also consistent with the neutrino oscillation parameters reported here.

\section{ATMOSPHERIC NEUTRINOS}

To carry out detailed studies of neutrino oscillations using atmospheric neutrinos, it is important to know the expected flux without neutrino oscillations. The difficulties and the uncertainties in the calculation of atmospheric neutrino fluxes differ between high and low energies. For low energy neutrinos around $1 \mathrm{GeV}$, the primary fluxes of cosmic ray components are relatively well known. Low energy cosmic ray fluxes of less than about $10 \mathrm{GeV}$ are modulated by solar activity, with the minimum flux occurring at times of high solar activity. At these energies, the primary cosmic rays are also affected by the geomagnetic field through a rigidity (momentum/charge) cutoff. For high energy neutrinos, above $100 \mathrm{GeV}$, primary cosmic rays with energies greater than $1000 \mathrm{GeV}$ are relevant. At these energies, solar activity and the rigidity cutoff do not affect the cosmic rays, but details of the higher energy primary cosmic ray flux are not as well measured.

There are several flux calculations $[21,22,23,24,25,26$, 27, 28, 29]. Unlike older calculations [30, 31], in which the secondary particles were assumed to travel in the direction of the primary cosmic ray (1-dimensional calculations), the current calculations employ three dimensional Monte Carlo methods. We outline below the methods of the calculation. We compared results from three atmospheric neutrino flux calculations [25, 28, 29] which cover the energy range relevant to the present analysis. The flux from Honda et al. [28] is used for the main numbers and figures quoted for the SuperKamiokande analysis.

Calculations start with primary cosmic rays based on measured fluxes, and include solar modulation and geomagnetic field effects. The interaction of cosmic ray particles with the air nucleus, the propagation and decay of secondary particles are simulated. We used a neutrino flux calculated specifically for the Kamioka site. According to the cosmic ray proton, helium and neutron measurements [32, 33], the cosmic ray flux was near that of solar minimum until the summer of 1999, rapidly decreased during the next year, and was at the minimum value consistent with solar maximum from summer of 2000 until Super-Kamiokande stopped taking date in July 2001. Therefore, the atmospheric neutrino Monte Carlo is calculated for 3 years of solar minimum, 1 year of changing ac- tivity, and 1 year of solar maximum.
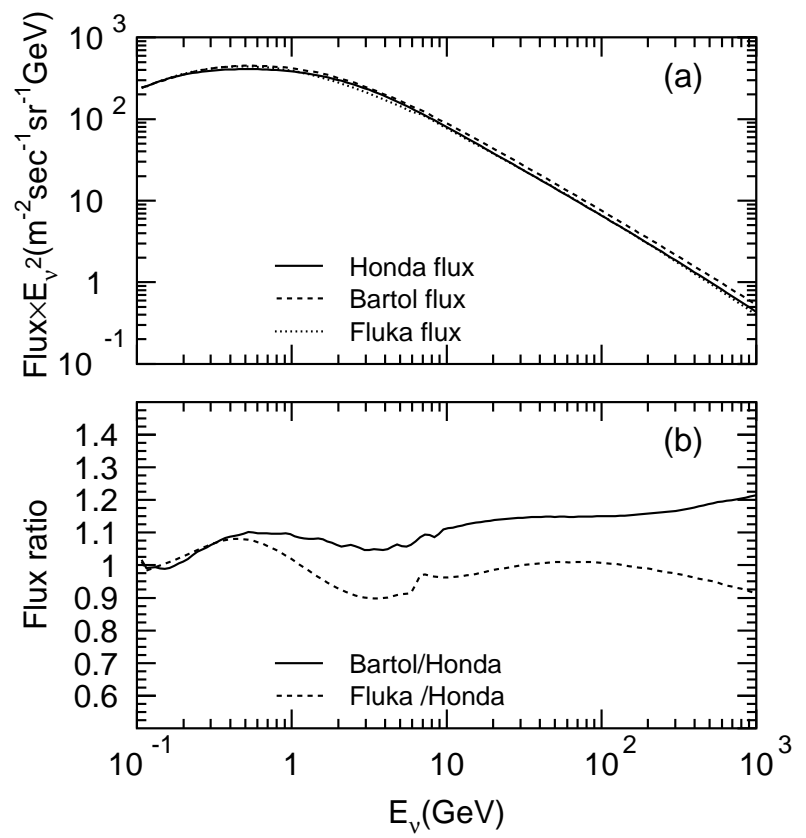

FIG. 2: (a) The direction averaged atmospheric neutrino energy spectrum for $v_{\mu}+\bar{v}_{\mu}$ calculated by several authors are shown by solid line [28], dashed line [29] and dotted line [25]. (b) The ratio of the calculated neutrino flux. The fluxes calculated in 29] (solid line) and [25] (dashed line) are normalized by the flux in [28].

The calculated energy spectra of atmospheric neutrinos at Kamioka are shown in Fig. 2]a). Also shown in Fig. 2(b) is the comparison of the calculated fluxes as a function of neutrino energy. The agreement among the calculations is about $10 \%$ below $10 \mathrm{GeV}$. This can be understood because the accuracy in recent primary cosmic ray flux measurements [34, 35] below $100 \mathrm{GeV}$ is about $5 \%$ and because hadronic interaction models used in each calculation are different.

However the primary cosmic ray data are much less accurate above $100 \mathrm{GeV}$. Therefore, for neutrino energies much higher than $10 \mathrm{GeV}$, the uncertainties in the absolute neutrino flux could be substantially larger than the disagreement level among the calculations. In Ref. [36], the authors discussed that the fit to the low energy $(<100 \mathrm{GeV})$ proton spectra gave a spectrum index of -2.74+-0.01. However, this spectrum does not fit well to the high energy data. Therefore, authors in Ref. [28] fit the high energy data allowing a different spectral index above $100 \mathrm{GeV}$ and found the best fit value of -2.71 . There is 0.03 difference in the spectrum index for low energy $(<100 \mathrm{GeV})$ and high energy $(>100 \mathrm{GeV})$ protons. Also, it is discussed in Ref. [36] that the spectrum index for the He flux can be fit by either -2.64 or -2.74 . There could be 0.10 uncertainty in the spectrum index for He. The spectrum indices for heavier nuclei have uncertainties larger than 0.05 [36]. Taking the flux weighted average of these spectrum index uncertainties, we assign 0.03 and 0.05 for the uncertainties in the energy spectrum index in the primary cosmic ray energy spectrum below and above $100 \mathrm{GeV}$, respectively.

Figure 3 shows the calculated flux ratio of $v_{\mu}+\bar{v}_{\mu}$ to $v_{e}+\bar{v}_{e}$ 
as a function of the neutrino energy, integrated over solid angle. This ratio is essentially independent of the primary cosmic ray spectrum. Especially in the neutrino energy region of less than about $5 \mathrm{GeV}$, most of the neutrinos are produced by the decay chain of pions and the uncertainty of this ratio is about $3 \%$, which is estimated by comparing the three calculation results. The contribution of $K$ decay in neutrino production is more important in the higher energy region; about $10 \%$ for $\mathrm{v}_{e}+\overline{\mathrm{v}}_{e}$ and $20 \%$ for $\mathrm{v}_{\mu}+\overline{\mathrm{v}}_{\mu}$ at $10 \mathrm{GeV}$. It increases to more than $30 \%$ at $100 \mathrm{GeV}$ for both $v_{e}+\bar{v}_{e}$ and $\nu_{\mu}+\bar{v}_{\mu}$. There, the ratio depends more on the $K$ production cross sections and the uncertainty of the ratio is expected to be larger. A $20 \%$ uncertainty in the $K / \pi$ production ratio [31, 37] causes at least a few percent uncertainty in the $v_{\mu}+\bar{v}_{\mu}$ to $v_{e}+\bar{v}_{e}$ ratio in the energy range of 10 to $100 \mathrm{GeV}$. However, as seen from Fig. 3. the difference in the calculated $v_{\mu}+\bar{v}_{\mu}$ to $v_{e}+\bar{v}_{e}$ ratio is as large as $10 \%$ at $100 \mathrm{GeV}$. As a consequence, above $5 \mathrm{GeV}$, we assumed that the uncertainty linearly increases with $\log E_{\mathrm{V}}$ from $3 \%$ at $5 \mathrm{GeV}$ to $10 \%$ at $100 \mathrm{GeV}$.

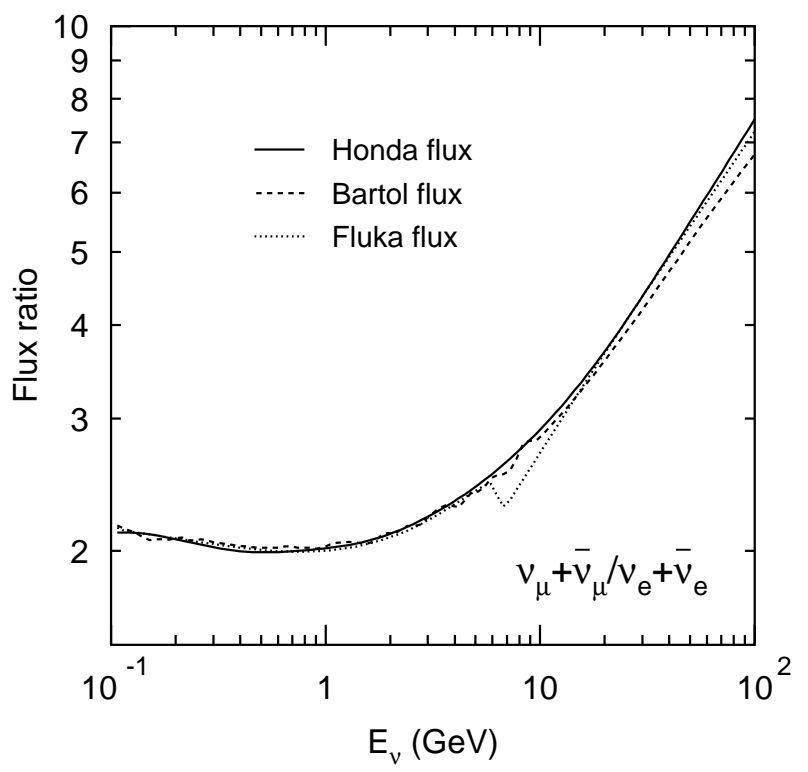

FIG. 3: The flux ratio of $v_{\mu}+\bar{v}_{\mu}$ to $v_{e}+\bar{v}_{e}$ averaged over all zenith and azimuth angles versus neutrino energy. Solid, dashed and dotted lines show the prediction by [28], [29] and [25], respectively.

Figure 4 shows the calculated flux ratios of $v_{\mu}$ to $\bar{v}_{\mu}$ and $v_{e}$ to $\bar{v}_{e}$. The calculations agree to about $5 \%$ for both of these ratios below $10 \mathrm{GeV}$. However, the disagreement gets larger above $10 \mathrm{GeV}$ as a function of neutrino energy. The systematic errors in the $v / \bar{v}$ ratio are assumed to be $5 \%$ below $10 \mathrm{GeV}$ and linearly increase with $\log E_{v}$ to $10 \%$ and $25 \%$ at $100 \mathrm{GeV}$, for the $v_{e}$ to $\bar{v}_{e}$ and $v_{\mu}$ to $\bar{v}_{\mu}$ ratios, respectively.

Figure 5 shows the zenith angle dependence of the atmospheric neutrino fluxes for several neutrino energies. At low energies, and at the Kamioka location, the fluxes of downward-going neutrinos are lower than those of upwardgoing neutrinos. This is due to the deflection of primary cosmic rays by the geomagnetic field, roughly characterized by a minimum rigidity cutoff. For neutrino energies higher than a
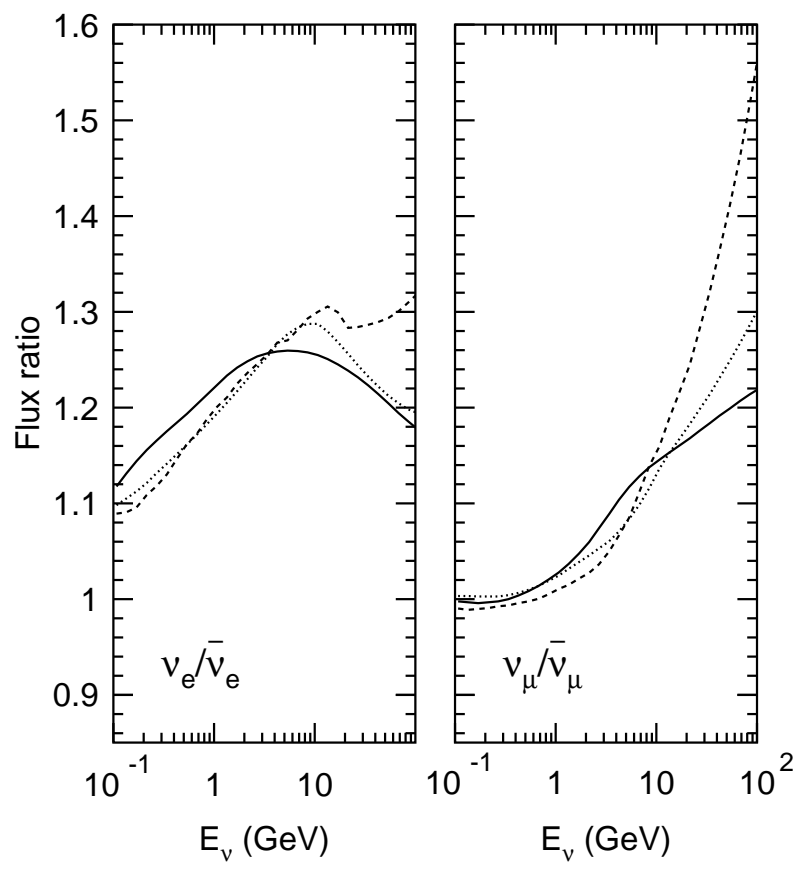

FIG. 4: The flux ratios of $v_{\mu}$ to $\bar{v}_{\mu}$ and $v_{e}$ to $\bar{v}_{e}$ versus neutrino energy. Solid, dashed and dotted lines show the prediction by [28], [29] and [25], respectively (same key as Fig. 3.

few $\mathrm{GeV}$, the calculated fluxes are essentially up-down symmetric, because the primary particles are more energetic than the rigidity cutoff.

The enhancement of the flux near horizon for low energy neutrinos is a feature characteristic of the three dimensional nature of the neutrino production in cosmic ray hadronic showers. This is properly treated in current flux calculations [21, 22, 23, 24, 25, 26, 27, 28, 29]. However, in Super-Kamiokande, the horizontal enhancement cannot be seen in the lepton zenith angle distribution, due to the relatively poor angular correlation between neutrinos and leptons below $1 \mathrm{GeV}$. The uncertainties in the up-down and verticalhorizontal ratios of the number of events are estimated by comparing the predicted ratios by various flux models. These uncertainties generally depend on the energy and the neutrino flavor. The uncertainty in the up-down event ratio is about 1 to $2 \%$ in the sub-GeV energy region and is about $1 \%$ in the multi-GeV energy region. The main source of the uncertainty in the vertical-horizontal ratio around $\mathrm{a} \mathrm{GeV}$ is the size of the horizontal enhancement of the flux due to the three dimensional effect; the uncertainty is estimated to be less than a few percent.

In the higher energy region, where upward through-going muons are relevant, the largest source of the uncertainty in the vertical-horizontal ratio is the $K$ production cross section. We assume that the $K / \pi$ production ratio uncertainty is $20 \%$ in the whole energy region [31, 37]. The uncertainties in the zenith angle and energy distributions due to the $K / \pi$ production uncertainty are included in the systematic errors in the analysis. This error is most important for higher energy neutrinos. For example, the vertical-horizontal uncertainty for 
upward through-going muons due to the $K / \pi$ production uncertainty is estimated to be $3 \%$ [38]. Figure 6 shows the zenith angle dependence of the atmospheric neutrino fluxes for higher energy region observed as upward muons in SuperKamiokande.
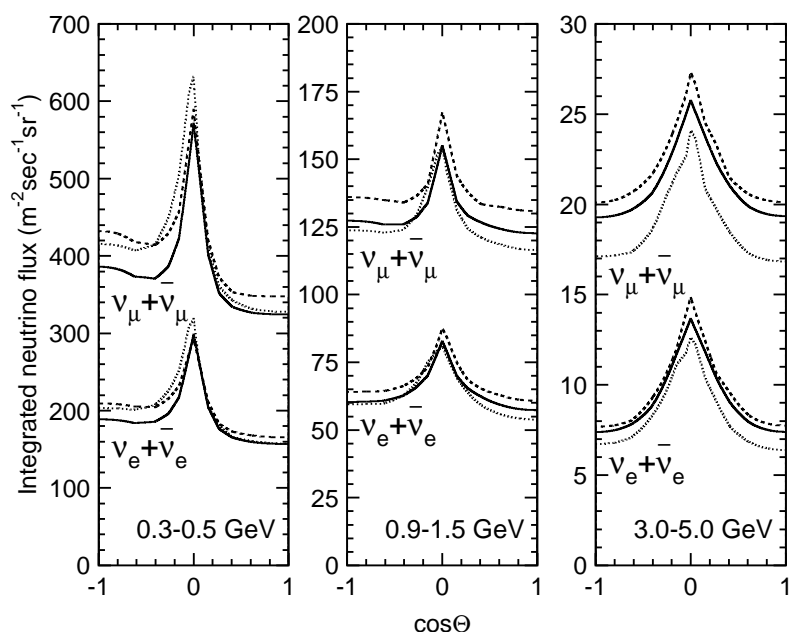

FIG. 5: The flux of atmospheric neutrinos versus zenith angle. Solid, dashed and dotted lines show the prediction by [28], [29] and [25], respectively (same key as Fig. 3.
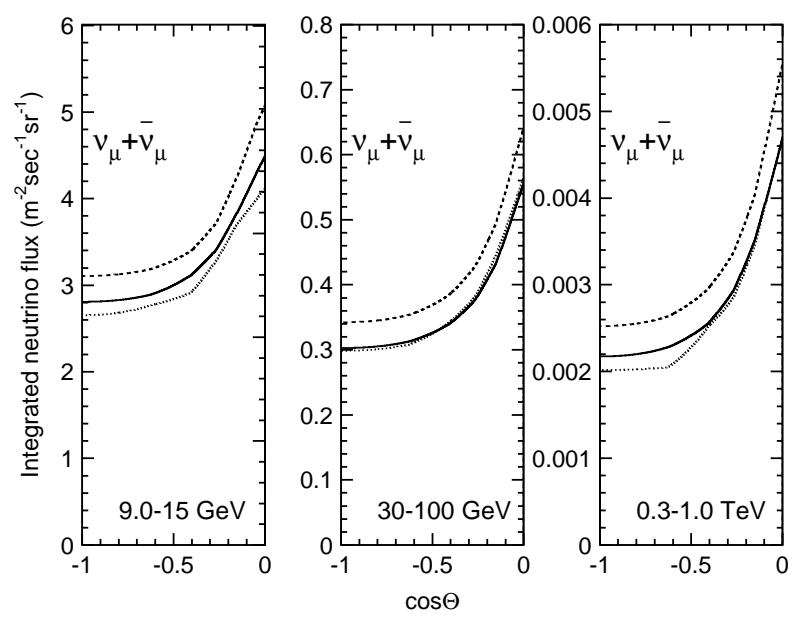

FIG. 6: The flux of upward-going atmospheric neutrinos versus zenith angle for higher energy region. Solid, dashed and dotted lines show the prediction by [28], [29] and [25], respectively (same key as Fig. 3.

The flight length of neutrinos is an important ingredient in the analysis of neutrino oscillation. For neutrinos passing a great distance through the Earth, the flight length can be accurately estimated. However, for horizontal and downward going neutrinos, the height of production in the upper atmosphere must be distributed by the Monte Carlo method. Figure 7 shows the calculated flight length distributions for vertically down-going and horizontal neutrinos.

In summary of the atmospheric neutrino flux, we remark that, the $\left(\mathrm{v}_{\mu}+\bar{v}_{\mu}\right)$ over $\left(\mathrm{v}_{e}+\bar{v}_{e}\right)$ flux ratio is predicted to an
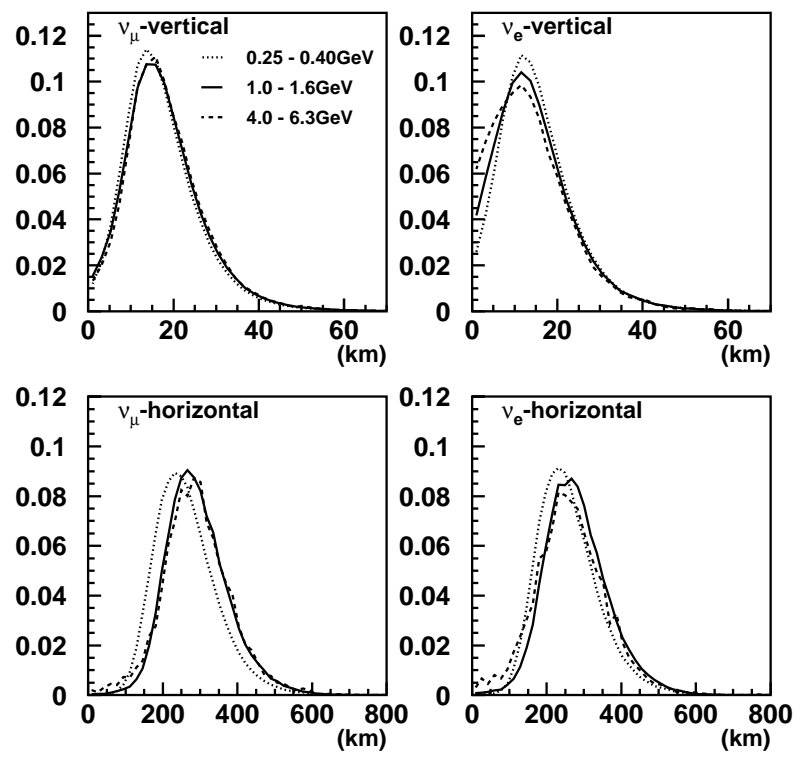

FIG. 7: The calculated flight length of neutrinos for vertically downgoing $(\cos \Theta=0.95-1.00)$ and near horizontal-going $(\cos \Theta=$ $0.05-0.10)$ directions. Distributions are made for both muonneutrinos and electron-neutrinos and for three energy intervals [28].

accuracy of about $3 \%$ in the energy region relevant to the data analysis discussed in this paper. The zenith angle dependence of the flux is well understood, and especially, above a few $\mathrm{GeV}$ neutrino energies, the flux is predicted to be up-down symmetric.

\section{THE SUPER-KAMIOKANDE DETECTOR}

Super-Kamiokande is a 50 kiloton water Cherenkov detector located at the Kamioka Observatory of the Institute for Cosmic Ray Research, University of Tokyo. Figure 8 shows a cut-away diagram of the Super-Kamiokande detector. This facility is in the Mozumi mine of the Kamioka Mining Company in Gifu prefecture, Japan, under the peak of Mt. Ikenoyama, providing a rock overburden of 2,700 m.w.e. Super-K consists of two concentric, optically separated water Cherenkov detectors contained in a stainless steel tank 42 meters high and 39.3 meters in diameter, holding a total mass of 50,000 tons of water. The inner detector (ID) is comprised of 11,146 Hamamatsu R3600 $50 \mathrm{~cm}$ diameter photomultiplier tubes (PMTs), viewing a cylindrical volume of pure water $16.9 \mathrm{~m}$ in radius and $36.2 \mathrm{~m}$ high. The $50 \mathrm{~cm}$ PMTs were specially designed [39] to have good single photoelectron (p.e.) response, with a timing resolution of $2.5 \mathrm{nsec}$ RMS. The ID is surrounded by the outer detector (OD), a cylindrical shell of water 2.6 to $2.75 \mathrm{~m}$ thick including a dead space $55 \mathrm{~cm}$. The OD is optically isolated from the ID, and is instrumented with 1,885 outward-facing Hamamatsu R1408 $20 \mathrm{~cm}$ PMTs, providing both a $4 \pi$ active veto and a thick passive radioactivity shield. The information from the outer detector is used to identify both incoming and outgoing muons.

Both ID and OD PMT signals are processed by asyn- 


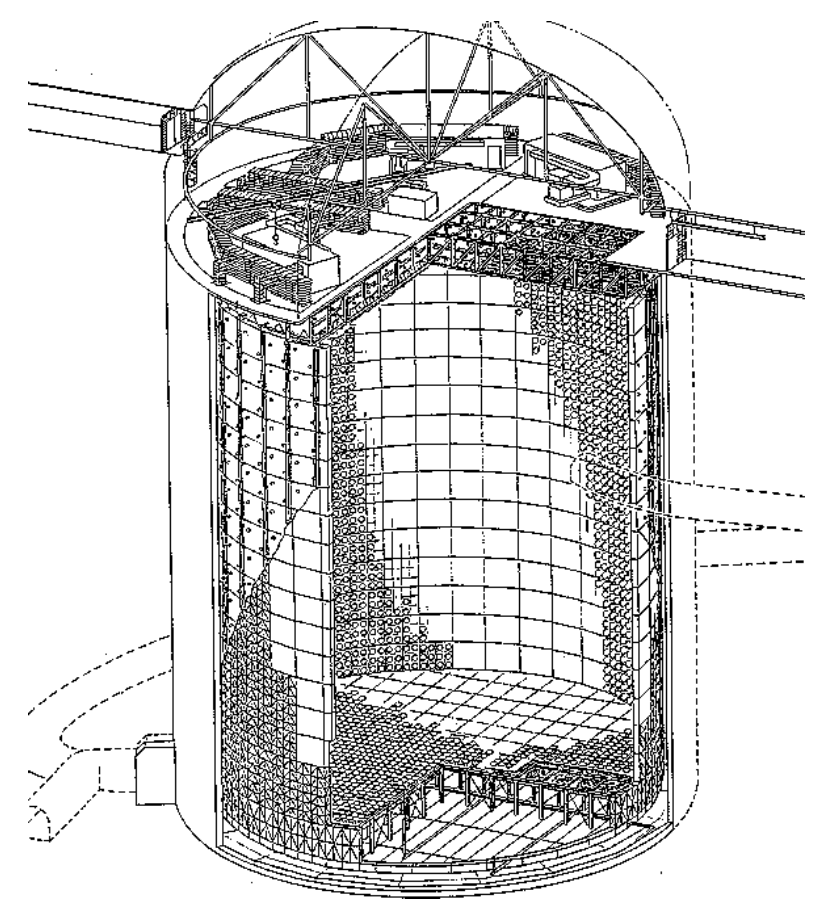

FIG. 8: A drawing of the Super-Kamiokande detector. The cutaway shows the inside lined with photomultiplier tubes comprising a photocathode coverage of about $40 \%$. The support structure is stainless steel beams. The thin outer region is shown with sparser density of outward facing PMTs: 2 outer PMTs for every unit of $3 \times 4$ inner PMTs. The top of the detector, under the hemisphere, consists of electronics huts and open work area.

chronous, self-triggering circuits that record the time and charge of each PMT hit over a threshold. Each ID PMT signal is digitized with custom Analog Timing Modules (ATMs) [40, 41] which provide $1.2 \mu \mathrm{sec}$ timing range at $0.3 \mathrm{nsec}$ resolution and $550 \mathrm{pC}$ charge range at $0.2 \mathrm{pC}$ resolution $(\sim 0.1$ p.e. $)$. The ATM has automatically-switched dual channels to provide deadtime-free data acquisition. The outer PMT signals are processed with custom charge-to-time conversion modules and digitized with LeCroy 1877 multi-hit TDCs over a $-10 \mu \mathrm{sec}$ to $+6 \mu \mathrm{sec}$ window centered on the trigger time. More details of the Super-K detector can be found in [42].

An event used in the atmospheric neutrino analysis is triggered by the coincidence of at least 30 PMT hits in a $200 \mathrm{nsec}$ window. The hit threshold for each individual PMT is about $1 / 4$ p.e. This trigger condition corresponds to the mean number of hit PMTs for a $5.7 \mathrm{MeV}$ electron. The trigger rate is $10-12 \mathrm{~Hz}$. The trigger rate due to cosmic ray muons is $2.2 \mathrm{~Hz}$. Digitized data are saved at a total rate of $12 \mathrm{~GB}$ per day.

The detector is simulated with a Monte Carlo program based on the GEANT package [43], in which the propagation of particles, the generation and propagation of Cherenkov photons, and the response of the PMTs is considered. For hadronic interactions in water, the CALOR package [44] was employed in our simulation code. This package is known to reproduce the pion interactions well down to low momentum regions of $\sim 1 \mathrm{GeV} / c$. For still lower momenta $\left(p_{\pi} \leq 500 \mathrm{MeV} / c\right)$, a custom program based on experimental data from $\pi-{ }^{16} \mathrm{O}$ scattering [45] and $\pi-p$ scattering [46] was used in our simulation code.

In connection with the propagation of charged particles, Cherenkov photons are generated. For the propagation of Cherenkov photons in water, Rayleigh scattering, Mie scattering and absorption were considered in our simulation code. The attenuation coefficients used were tuned to reproduce the measurement using laser system (Section tion and absorption on detector material, such as the surface of PMTs and black plastic sheets between the PMTs was simulated based on direct measurements, using probability functions that depend on the photon incident angle.

\section{A. Calibration of the Super-Kamiokande Detector}

Water transparency was measured using a dye laser beam injected into detector water at wavelengths of 337, 371, 400, and $420 \mathrm{~nm}$. From the spatial and timing distribution of observed laser light, both absorption and scattering coefficients were studied and incorporated into our detector simulator. The water transparency was continuously monitored using cosmic ray muons as a calibration source.

The accuracy of the absolute energy scale was estimated to be $\pm 1.8 \%$ based on the following calibration sources: the total number of photo-electrons as a function of muon track length, where the muon track length is estimated by the reconstructed muon entrance point and the reconstructed vertex point of an electron from the muon-decay; the total number of photo-electrons as a function of Cherenkov angle for low energy cosmic ray muons; the spectrum of muon-decay electrons; and the invariant mass of $\pi^{0} \mathrm{~s}$ produced by neutrino interactions (Figure 9. Figure 10 summarizes the absolute energy scale calibration by these studies. The stability of the energy scale was also monitored continuously using stopping muons and muon-decay electrons. Figure 11 shows the time variation of the mean reconstructed energy of stopping muons divided by muon range and the mean reconstructed electron energy from muon-decays. The R.M.S of the energy scale variation is $\pm 0.9 \%$ over the time of the experiment. From combining the absolute energy scale accuracy study $( \pm 1.8 \%)$ and the energy scale time variation $( \pm 0.9 \%)$, the total uncertainty of the energy scale of atmospheric neutrino detection was estimated to be $\pm 2.0 \%$.

The uniformity of the detector response was studied by decay electrons from stopping cosmic ray muons and neutrino induced $\pi^{0}$. Both are good calibration sources because the vertex position is distributed in the fiducial volume and the momentum distribution is nearly uniform in all directions. To account for muon polarization in the estimation of the zenith and azimuthal angle dependence of the detector gain, only electrons decaying in the direction perpendicular to the initial muon direction are used. This condition is $-0.25<\cos \Theta_{e \leftrightarrow \mu}<0.25$ where $\Theta_{e \leftrightarrow \mu}$ is the opening angle between the electron and muon directions. Using the selected electrons, the mean of the reconstructed momentum of the electrons are plotted as a function of the zenith angle of the electrons in Figure 12-(a). From the figure, the detector gain 


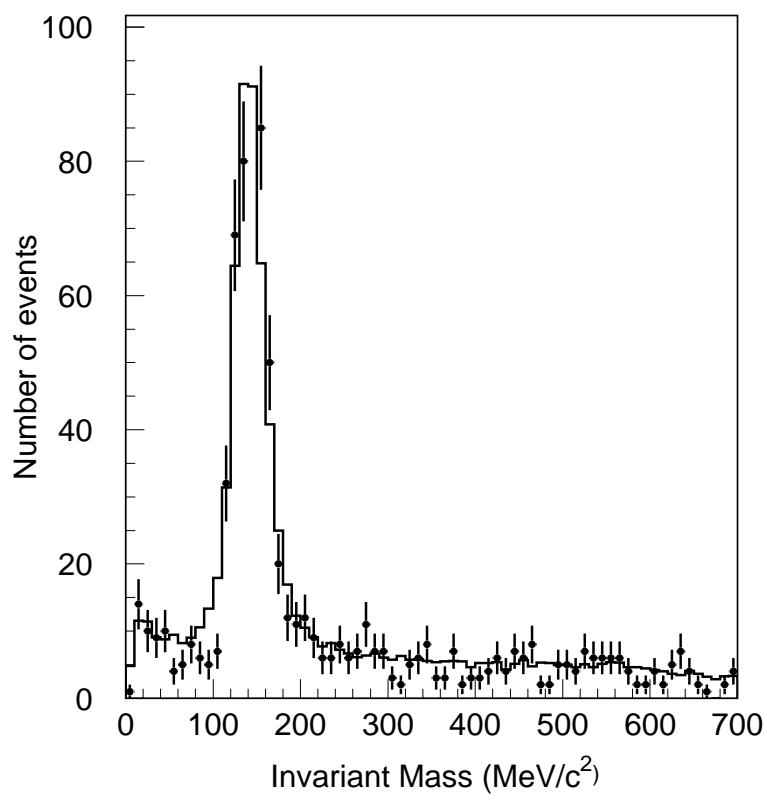

FIG. 9: The invariant mass distribution of fully-contained events with two $e$-like rings and no muon-decay electron, for SK data (points) and atmospheric neutrino Monte Carlo (histogram). A peak from neutrino induced $\pi^{0}$ is clearly observed.

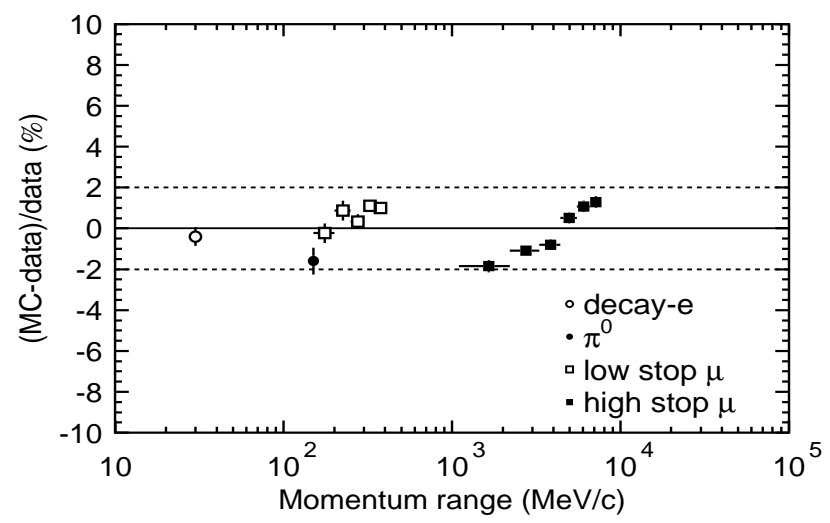

FIG. 10: The determination of the absolute energy scale of SuperKamiokande based on in situ calibration with $\mu$-decay electrons, $\pi^{0} \rightarrow \gamma \gamma$ invariant mass, and the Cherenkov light of stopping cosmic ray muons.

was uniform over all zenith angles within $\pm 0.6 \%$. Figure 12-(b) shows the azimuthal angle dependence of the reconstructed momentum. Again, the detector gain is uniform over all azimuthal angles within $\pm 1 \%$. Finally, Figure 13 shows the zenith angle dependence of the reconstructed $\pi^{0}$ mass. This figure also suggests that the detector gain was uniform over all zenith angles within $\pm 1 \%$.



FIG. 11: The mean reconstructed energy of cosmic ray stopping muons divided by their range (upper) and muon-decay electron (lower) as a function of elapsed days. Vertical axes in both figures are normalized to mean values and each data point corresponds to two month period. The variation is within $\pm 2 \%$.
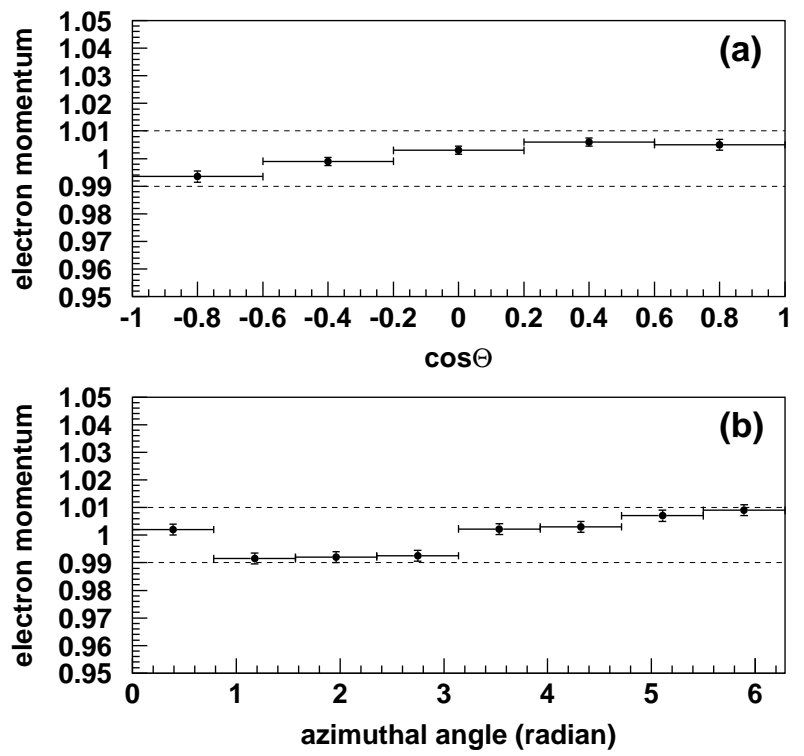

FIG. 12: The gain uniformity of the Super-Kamiokande detector as determined by the mean value of the reconstructed decay electron momentum (a) as a function of zenith angle, and (b) as a function of azimuthal angle. Vertical axes in both figures are normalized to the mean values.

\section{ATMOSPHERIC NEUTRINO MONTE CARLO}

The result published in this paper relies heavily on detailed comparison of the experimental data with the theoretical expectation. An important element of this is to simulate the interaction of neutrinos from $10 \mathrm{MeV}$ to $100 \mathrm{TeV}$ with the nuclei of water, or in the case of upward muons, the nuclei of the rock surrounding the detector, assumed to be "standard rock" $(\mathrm{Z}=11, \mathrm{~A}=22)$. Therefore, we have developed two Monte Carlo models designed to simulate neutrino interac- 


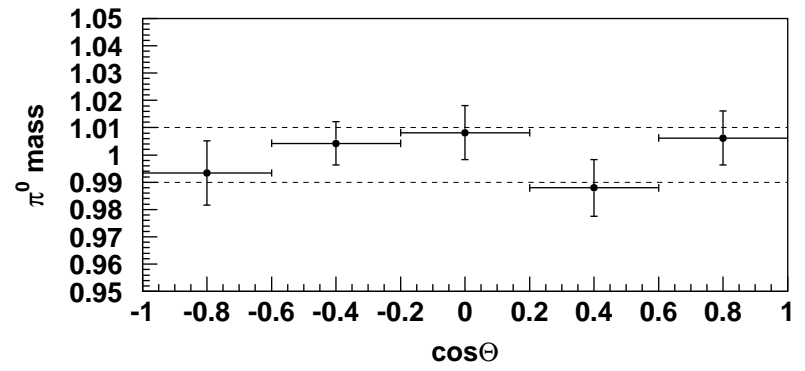

FIG. 13: The gain uniformity of the Super-Kamiokande detector as determined by the fitted peak of the $\pi^{0} \rightarrow \gamma \gamma$ mass distribution as a function of zenith angle. Vertical axis is normalized to the mean value.

tions with protons, oxygen and sodium [47, 48]. Both models use similar input physics models. Here, one of the models [47] (NEUT) will be described. The detailed description for the other model (NUANCE) can be found elsewhere [48].

In the simulation program, the following charged and neutral current neutrino interactions are considered:

- (quasi-)elastic scattering, $v N \rightarrow l N^{\prime}$,

- single meson production, $v N \rightarrow l N^{\prime} m$,

- coherent $\pi$ production, $v^{16} \mathrm{O} \rightarrow l \pi^{16} \mathrm{O}$,

- deep inelastic scattering, $v N \rightarrow l N^{\prime}$ hadrons.

Here, $N$ and $N^{\prime}$ are the nucleons (proton or neutron), $l$ is the lepton, and $m$ is the meson, respectively. For single meson production, $K$ and $\eta$ production are simulated as well as the dominant $\pi$ production processes. If the neutrino interaction occurred in the oxygen nuclei, generated particles like pions and kaons interact with the nucleus before escaping.

\section{A. Elastic and quasi-elastic scattering}

The formalization of quasi-elastic scattering off a free proton, which was used in the simulation programs, was described by Llewellyn-Smith[49]. For scattering off nucleons in ${ }^{16} \mathrm{O}$, the Fermi motion of the nucleons and Pauli Exclusion Principle were taken into account. The nucleons are treated as quasi-free particles using the relativistic Fermi gas model of Smith and Moniz[50]. The momentum distribution of the nucleons were assumed to be flat up to the fixed Fermi surface momentum of $225 \mathrm{MeV} / c$. This Fermi momentum distribution was also used for other nuclear interactions. The nuclear potential was set to $27 \mathrm{MeV} / c$.

\section{B. Single meson production}

Rein and Sehgal's model was used to simulate the resonance productions of single $\pi, K$ and $\eta[51$, 52]. In this method, the interaction is separated into two parts:

$$
\begin{aligned}
v+N & \rightarrow l+N^{*}, \\
N^{*} & \rightarrow m+N^{\prime},
\end{aligned}
$$

where $m$ is a meson, $N$ and $N^{\prime}$ are nucleons, and $N^{*}$ is a baryon resonance. The hadronic invariant mass, $W$, the mass of the intermediate baryon resonance, is restricted to be less than $2 \mathrm{GeV} / c^{2}$. In addition to the dominant single $\pi$ production, $K$ and $\eta$ production is considered. The production of $\eta$ is evidently much smaller than $\pi$, as seen in Fig. 9 where there is no evidence for a mass peak near $549 \mathrm{MeV} / \mathrm{c}^{2}$ in data or Monte Carlo.

To determine the angular distribution of pions in the final state, we also use Rein and Sehgal's method for the $P_{33}(1232)$ resonance. For the other resonances, the directional distribution of the generated pions is set to be isotropic in the resonance rest frame. The angular distribution of $\pi^{+}$has been measured for $v p \rightarrow \mu^{-} p \pi^{+}[53]$ and the results agree well with the Monte Carlo prediction. We also consider the Pauli blocking effect in the decay of the baryon resonance by requiring that the momentum of nucleon should be larger than the Fermi surface momentum. Pion-less delta decay is also considered, where $20 \%$ of the events do not have the pion and only the lepton and nucleon are generated [54].

The quasi-elastic and single meson production models have a parameter (axial vector mass, $M_{A}$ ) that must be determined by experiments. For larger $M_{A}$ values, interactions with higher $Q^{2}$ values (and therefore larger scattering angles) are enhanced for these channels. The $M_{A}$ value was tuned using the K2K [19] near detector data. In our atmospheric neutrino Monte Carlo simulation, $M_{A}$ is set to $1.1 \mathrm{GeV}$ for both the quasi-elastic and single-meson production channels, but the uncertainty of the value is estimated to be $10 \%$. Figure 14 shows the K2K 1 kton water Cherenkov data on the scattering angle for single Cherenkov ring events [19] together with the prediction by the Monte Carlo used in this analysis. The scattering angle agrees well between the data and Monte Carlo overall, although the suppression of events at small angle is being studied by several groups [55].

Coherent single-pion production, the interaction between the neutrino and the entire oxygen nucleus, is simulated using the formalism developed by Rein and Sehgal [56].

\section{Deep inelastic scattering}

In order to calculate the cross-sections of deep inelastic scattering, the GRV94 [57] parton distribution function is used. In the calculation, the hadronic invariant mass, $W$, is required to be greater than $1.3 \mathrm{GeV} / c^{2}$. However, the multiplicity of pions is restricted to be greater than or equal to 2 for $1.3<W<2.0 \mathrm{GeV} / c^{2}$, because single pion production is separately simulated as previously described. In order to generate events with multi-hadron final states, two models are used. For $W$ between 1.3 and $2.0 \mathrm{GeV} / \mathrm{c}^{2}$, a custom-made program [58] is used to generate the final state hadrons; only pions are considered in this case. For $W$ larger than $2 \mathrm{GeV} / c^{2}$, PYTHIA/JETSET [59] is used.

Total charged current cross sections including quasi-elastic scattering, single meson productions and deep inelastic scattering are shown in Fig 15 


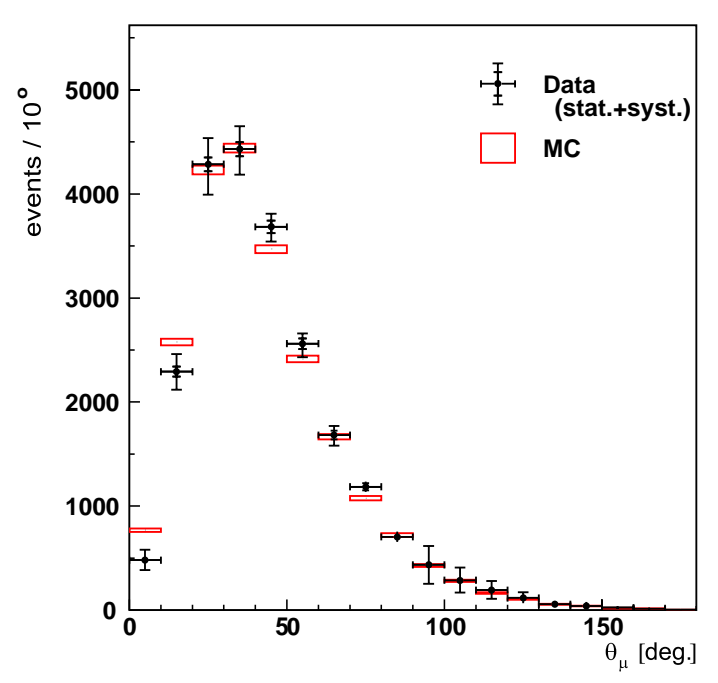

FIG. 14: The scattering angle distribution by neutrino interactions off the $\mathrm{H}_{2} \mathrm{O}$ target from the K2K experiment (data are from Fig.1(b) of Ref. [19]). Single Cherenkov ring events observed by the 1 kton water Cherenkov detector are used. The histogram shows the prediction by the Monte Carlo used in the present analysis.

\section{Nuclear effects}

The interactions of mesons within the ${ }^{16} \mathrm{O}$ nucleus are also important for the atmospheric neutrino analysis. Basically, all of the interactions are treated by using a cascade model. The interactions of pions are very important because the cross section for pion production is quite large for neutrino energies above $1 \mathrm{GeV}$ and the interaction cross sections for pions in nuclear matter is also large.

In our simulation program, we consider the following pion interactions in ${ }^{16} \mathrm{O}$ : inelastic scattering, charge exchange and absorption. The procedure to simulate these interactions is as follows. The initial position of the pion generated according to the Woods-Saxon nucleon density distribution[72]. The interaction mode is determined from the calculated mean free path of each interaction. To calculate the mean free path, we adopt the model described by Salcedo et al. [73]. The calculated mean free path depends not only on the momentum of the pion but also on the position of the pion in the nucleus. If inelastic scattering or charge exchange occurs, the direction and momentum of the pion are determined by using the results of a phase shift analysis obtained from $\pi-N$ scattering experiments [74]. When calculating the pion scattering amplitude, the Pauli blocking effect is also taken into account by requiring the nucleon momentum after interaction to be larger than the Fermi surface momentum at the interaction point. The pion interaction simulation was checked using data for the following three interactions: $\pi^{12} \mathrm{C}$ scattering, $\pi^{16} \mathrm{O}$ scattering and pion photo-production $\left(\gamma+{ }^{12} \mathrm{C} \rightarrow \pi^{-}+X\right)[75]$.
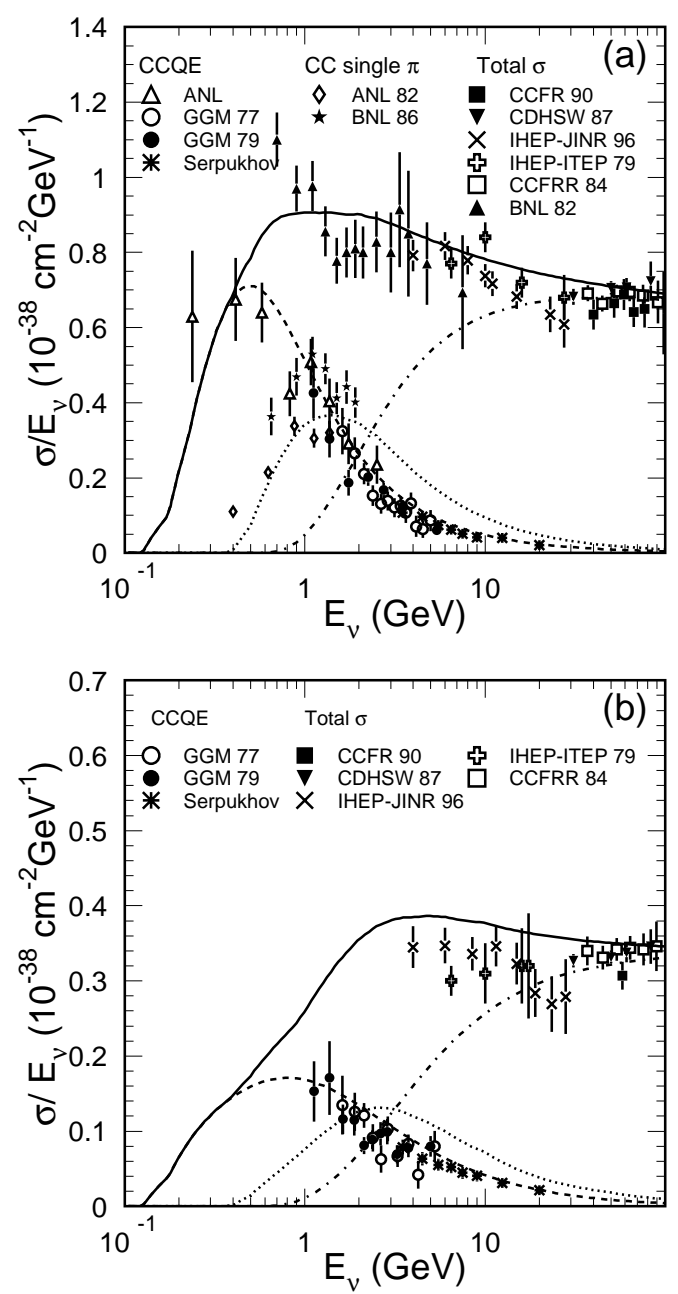

FIG. 15: Charged current total cross section divided by $E_{v}$ for (a) neutrino and (b) anti-neutrino nucleon charged current interactions. Solid line shows the calculated total cross section. The dashed, dot and dash-dotted lines show the calculated quasi-elastic, single-meson and deep-inelastic scatterings, respectively. Data points are taken from the following experiments: $(\triangle)$ ANL 60], $(\bigcirc)$ GGM77|61], $(\bullet) G G M 79$ (a) 62],(b) [63], (*)Serpukhov [64], $(\diamond)$ ANL82|65],

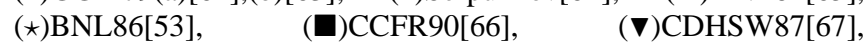
( $\times$ IHEP-JINR96[68], (+)IHEP-ITEP79 [69], (口)CCFRR84[70], and $(\mathbf{\Lambda})$ BNL82[71].

\section{SUPER-KAMIOKANDE DATA}

The Super-Kamiokande data set was acquired from May, 1996 to July, 2001. Three separate data reduction paths were used to isolate samples of fully-contained events, partiallycontained events, and upward-going muons. The fullycontained and partially-contained data sets shared a common set of good run selection criteria, and have identical live-time. The upward-going muon data set relies mostly on fitting long muon track directions; it was less susceptible to detector effects, and therefore had looser data quality cuts and somewhat higher live-time. 
To separate fully-contained and partially-contained events, a fast spatial clustering algorithm was applied to the outer detector hits; if the number of hits in the largest OD cluster was less than 10, the event was defined as fully-contained (FC), otherwise, it was defined as partially-contained (PC). Figure 16 shows the number of the outer detector hits in the largest OD cluster. A clear separation of FC and PC events is seen at 10 hits. The systematic uncertainty of the FC and PC separation was estimated by scaling the number of outer detector hits to match the distribution among data and MC.

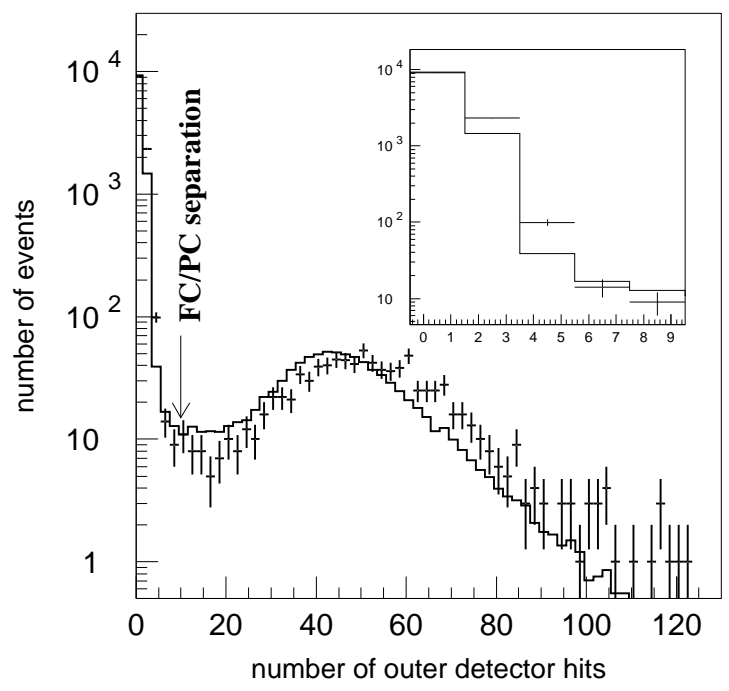

FIG. 16: The number of hits in the largest outer detector cluster, which is used to separate the fully-contained and partially-contained event samples. The histogram shows the MC prediction with neutrino oscillations.

In the early stage of the Super-Kamiokande experiment, two data analyses based on independent data reduction, reconstruction, and simulation were carried out to make sure that the atmospheric neutrino results did not have any serious mistakes [1, 2]. After confirming that the two analyses described atmospheric neutrino results equally well, they were unified. This paper is based on the unified analysis plus methods developed since that time.

\section{A. Fully-Contained Events}

\section{Data Reduction}

The Super-Kamiokande event sample consists mainly of downward-going cosmic ray muons and low energy radioactivity from parents such as radon. Owing to the double structure of the inner and outer detectors, cosmic ray muons are easily removed with high efficiency by requiring little or no activity in the outer detector. For atmospheric neutrino analysis, we then consider only events with visible energy above $30 \mathrm{MeV}$, where visible energy $\left(E_{v i s}\right)$ is defined as the energy of an electromagnetic shower that gives a certain amount of
Cherenkov light (for example, a muon of momentum 300 $\mathrm{MeV} / c$ yields a visible energy of about $110 \mathrm{MeV}$ ). To make the final FC data sample, five steps of data reduction criteria were used:

(i-ii) Simple and efficient criteria were applied in the first and second reduction steps: (1) the total charge collected in the inner detector within a $300 \mathrm{nsec}$ time window must be greater than 200 p.e.'s; (2) the ratio of the maximum p.e. in any single ID PMT to the total number of p.e.'s in the inner detector must be less than 0.5 ; (3) the number of hits in the outer detector within an $800 \mathrm{nsec}$ time window should be less than 25 hits; (4) the time interval from the preceding event should be greater than $100 \mu \mathrm{sec}$, to reject electrons from stopping muon decays.

(iii) More complex criteria were applied in the third reduction step with the help of event reconstruction tools, for further rejection of cosmic ray muons and low energy events: (1) no spatial cluster of more than 10 OD PMT hits is allowed within $8 \mathrm{~m}$ from the entrance or exit point of a candidate muon track fit to the inner detector light pattern, (2) the number of ID hits in $50 \mathrm{nsec}$ residual time window should be 50 hits or more.

(iv) In the fourth reduction step, additional selection criteria were used to eliminate spurious events, such as those due to "flashing" PMTs that emit light from internal corona discharges. Flasher events were removed by two different methods. (1) Typical flasher events have broader PMT timing distributions than the neutrino events. Events with broader timing distributions were eliminated. (2) Since flasher events have a tendency to be repeated with similar spatial hit distribution, the pattern information of observed charge was used to eliminate these events. A correlation parameter based on the charge pattern was calculated with other data events and a "matched" tag was assigned for highly correlated events. A cut was applied based on maximum correlation value and the number of "matched" with other events.

(v) Two further event types are eliminated in the fifth reduction step. (1) Events are removed which have $\geq 10$ OD hits in $200 \mathrm{nsec}$ coincidence preceding the trigger time ( $-8900 \sim-100 \mathrm{nsec}$ ); this eliminates decay electrons from invisible cosmic ray muons that are below Cherenkov threshold in the inner detector. (2) Cosmic ray muons are removed using a more precise fitter and the same criteria as (1) of (iii).

(vi) Finally, the vertex was required to be within a fiducial volume, 2 meters from the wall of the inner detector, and the visible energy was required to be greater than $30 \mathrm{MeV}$.

Table shows the number of events for each reduction step. Also shown are the the number of Monte Carlo events for each reduction step.

\section{Event Reconstruction}

The fully-contained events underwent a series of reconstruction steps in order to classify their origin and properties. First, the vertex position of an event was determined using PMT hit times; the point which best fit the distribution of PMT times (when adjusted for the time of flight of the Cherenkov light) was defined as the vertex position. This vertex was re- 


\begin{tabular}{l|c|c}
\hline \hline Reduction step & Data & Monte Carlo \\
\hline \hline Trigger & 1889599293 & $14013.9(100.00 \%)$ \\
First reduction & 4591659 & $14006.3(99.95 \%)$ \\
Second reduction & 301791 & $14006.1(99.94 \%)$ \\
Third reduction & 66810 & $13993.3(99.85 \%)$ \\
Fourth reduction & 26937 & $13898.1(99.17 \%)$ \\
Fifth reduction & 23984 & $13895.3(99.15 \%)$ \\
Fiducial volume and & 12180 & $13676.7(97.59 \%)$ \\
visible energy cuts & & \\
\hline \hline
\end{tabular}

TABLE I: Number of events after each reduction for fully-contained events during 1489 days of the detector live-time. The Monte Carlo numbers and efficiencies down to the fifth reduction are for events whose real vertex is in the fiducial volume, the number of outer detector hits fewer than 10 and the visible energy larger than $30 \mathrm{MeV}$. In the last line, the fitted vertex is used for both data and Monte Carlo.

constructed again after particle identification was established, to correct for particle track length. The vertex resolution was estimated to be $30 \mathrm{~cm}$ for single-ring fully-contained events. The distribution of vertex position for both data and MC as a function of the $z$-coordinate and $r^{2}$-coordinate are shown in Figures 17 (a) and (b), respectively. In these and several further figures, the original Monte Carlo prediction is modified by the oscillation of $C C v_{\mu}$ interactions according to the best-fit parameters $\left(\sin ^{2} 2 \theta=1.0, \Delta m^{2}=2.1 \times 10^{-3} \mathrm{eV}^{2}\right)$, as found in Section VI. Only a simple survival probability suppression is applied for these comparisons, the adjusted systematic terms that will be described in Section VI

After an initial ring direction and vertex were found by use of the timing method, a Hough transform [76] based technique was applied to automatically determine the number of Cherenkov rings in an event and their directions. The technique was iterative. A second ring was searched for by choosing possible ring directions based on the Hough map, and a likelihood technique was used to determine if a second ring from this list of possible rings was more consistent with the data than just one ring. If a second ring was found to be necessary, then this procedure was repeated as often as needed (to a maximum of 5 found rings), each time fixing the previously found rings, until finally no further rings were necessary to fit the data. Figure 18 shows the likelihood difference between the 2-ring assumption and a 1-ring assumption. A cut was made at likelihood difference of 0 to separate single and multi-ring events. The likelihood distributions, especially the one for multi-GeV energy region, have a slight difference in the peak positions between the data and the Monte Carlo. This difference is taken as a source of the systematic error in the measurements of the $v_{\mu}$ and $v_{e}$ rates. More details will be discussed later. Figure 19 shows the distribution of the number of reconstructed Cherenkov rings for both the data and $\mathrm{MC}$.

The efficiency for identifying charged current (CC) quasielastic $v_{e}\left(v_{\mu}\right)$ events as single-ring was $93.2(95.8) \%$, and the angular resolution for these single-ring events was estimated to be $3.0^{\circ}$ and $1.8^{\circ}$ for single-ring $e$-like and $\mu$-like events,
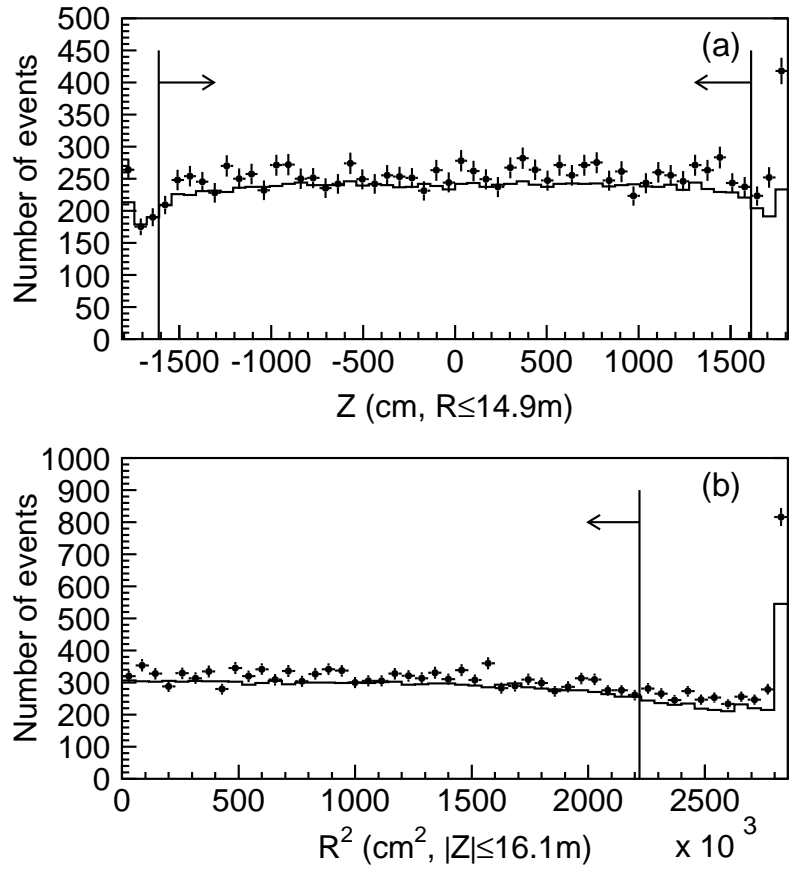

FIG. 17: The distribution of fully-contained event vertices in the (a) $z$-coordinate and (b) $r^{2}$-coordinate, comparing SK data and atmospheric neutrino Monte Carlo. The points show the data and the histogram shows the Monte Carlo prediction. The Monte Carlo includes neutrino oscillation with $\left(\sin ^{2} 2 \theta=1.00, \Delta m^{2}=2.1 \times 10^{-3} \mathrm{eV}^{2}\right)$.

respectively.

To determine the identity of the final state particles, a particle identification algorithm was applied which exploited systematic differences in the shape and the opening angle of Cherenkov rings produced by electrons and muons. Cherenkov rings from electromagnetic cascades exhibit a more diffuse light distribution than those from muons. Figures 20 and 21$]$ show observed single-ring $e$-like and $\mu$-like events, respectively. The opening angle of the Cherenkov cone, which depends on $\beta(\equiv v / c)$, was also used to separate electrons and muons at low momenta. The validity of the method was confirmed by a beam test experiment at KEK [77]. The misidentification probabilities for single-ring muons and electrons were estimated to be $0.7 \%$ and $0.8 \%$ respectively, using simulated $\mathrm{CC}$ quasi-elastic neutrino events. The distribution of the likelihood variable used to discriminate single-ring electrons and muons are shown for both the data and MC for the sub-GeV and multi-GeV samples in Figure 22 In both of these cases there is a clear separation of the likelihood variable.

Figure 23 shows the likelihood variable distribution for the brightest ring of FC multi-ring events. Due mostly to overlapping of Cherenkov photons from multiple particles, the separation of the particle type for a Cherenkov ring in a multi-ring event is not as good as that for a single-ring event.

The identification efficiency was checked using cosmic ray muons that stop in the detector and subsequently decay to electrons. These events are easily selected by their timing signature. The resulting misidentification probabilities for stop- 


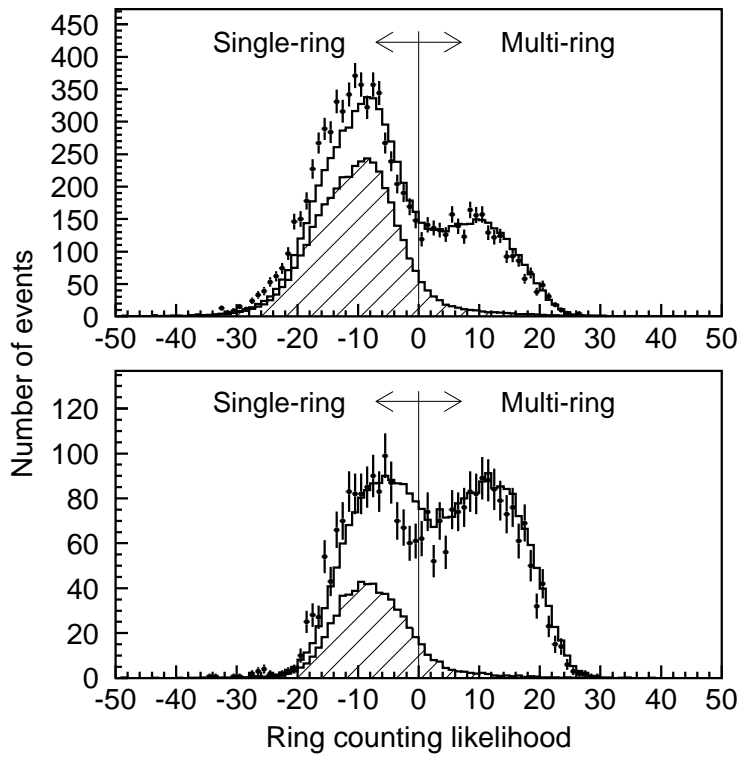

FIG. 18: The distribution of the likelihood difference between a single-ring assumption and a multi-ring assumption for sub-GeV (top) and multi-GeV (bottom) FC events. The points show the data and the histograms show the Monte Carlo prediction. The Monte Carlo includes neutrino oscillation with $\left(\sin ^{2} 2 \theta=1.00, \Delta m^{2}=\right.$ $2.1 \times 10^{-3} \mathrm{eV}^{2}$ ). The hatched histograms show the charged current quasi-elastic interactions.

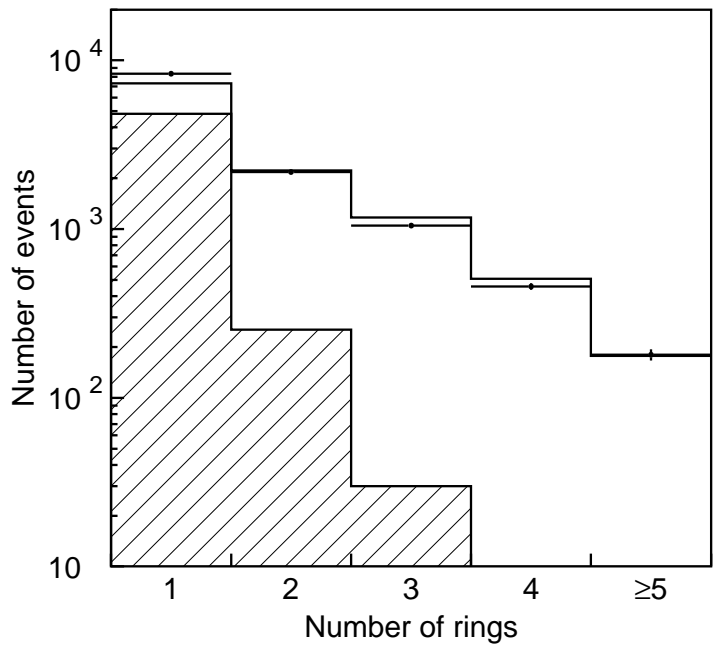

FIG. 19: The distribution of the number of identified Cherenkov rings, comparing SK data and atmospheric neutrino Monte Carlo. The Monte Carlo includes neutrino oscillation with $\left(\sin ^{2} 2 \theta=1.00\right.$, $\Delta m^{2}=2.1 \times 10^{-3} \mathrm{eV}^{2}$ ). The hatched histogram shows the charged current quasi-elastic interactions.

ping cosmic ray muons and decay electron light patterns were $0.4 \pm 0.1 \%$ and $1.8 \pm 0.5 \%$ respectively, in good agreement with the Monte Carlo estimates. This check was performed continuously during data-taking, and particle identification performance remained stable despite water transparency that varied from about $90 \mathrm{~m}$ to $120 \mathrm{~m}$.

Next, the Cherenkov rings were re-fit taking into account

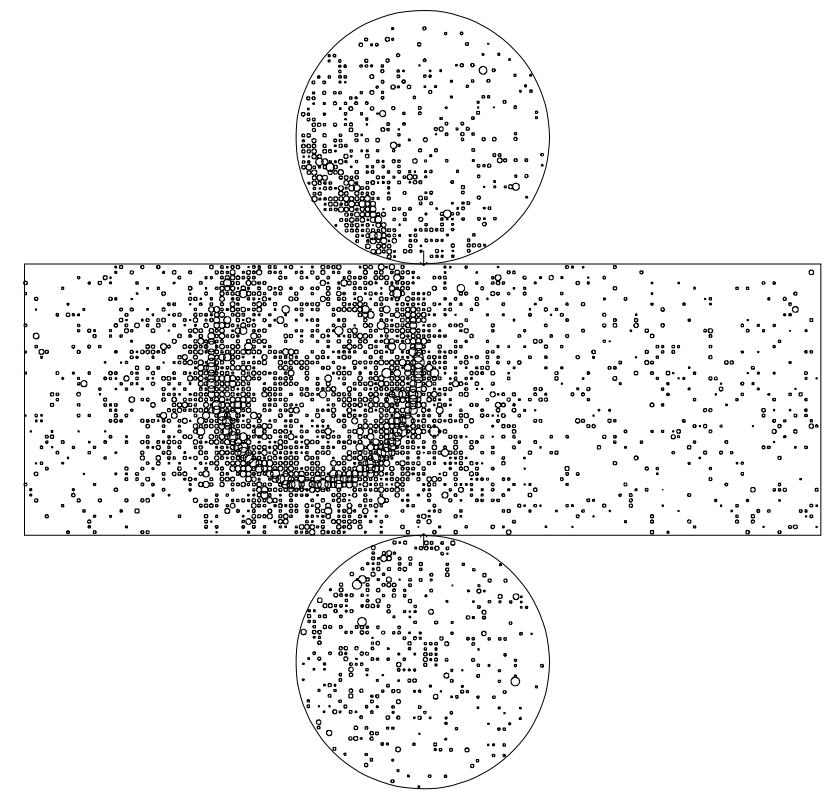

FIG. 20: An example event display of a single-ring $e$-like event. Each small circle represents a hit PMT and the size of the circle represents the number of photons to hit it. In this event the boundary of the Cherenkov light is smeared over many PMTs as the light comes from numerous positrons and electrons in the electromagnetic shower.

the expected light pattern given by the particle identification, and in the case of single-ring events, a specialized event fitter was applied. After the rings were re-fit and the total photo-electrons in the event were apportioned between all of the rings, each ring was assigned a momentum based on proportion of Cherenkov photons. The momentum of a particle was determined from the total number of p.e.'s within a $70^{\circ}$ half-angle cone relative to the track direction, with corrections for light attenuation and PMT angular acceptance. The resulting momentum resolution is estimated to be $0.6+2.6 / \sqrt{\mathrm{P}(\mathrm{GeV} / \mathrm{c})} \%$ for single-ring electrons and $1.7+0.7 / \sqrt{\mathrm{P}(\mathrm{GeV} / \mathrm{c})} \%$ for single-ring muons. A final procedure was performed which utilized the final energy and angle information of the rings to remove rings which were most likely not real.

Although decay electrons are not used in this oscillation analysis, they are a useful signature in other atmospheric neutrino analyses and the search for proton decay; therefore, we document their treatment here. Decay electrons were identified either as: (a) PMT hits within the same time window as the primary event trigger (up to $900 \mathrm{~ns}$ later) or (b) a later independent event trigger. In the first case, a sliding search window of width $30 \mathrm{~ns}$ began $100 \mathrm{~ns}$ after the primary trigger; a decay electron was counted if 40 hits were found in coincidence above the background level. In the second type, 60 hits were required in a $50 \mathrm{~ns}$ time window, and goodness-offit for a Cherenkov ring pattern is required. In both cases the vertex is known from the primary event and is used to subtract the time-of-flight of the Cherenkov light. If the decay occurs around $900 \mathrm{~ns}$, the hits may be split between the primary event trigger and a subsequent event trigger. In some 




FIG. 21: An example event display of a single-ring $\mu$-like event. In this event the boundary of the Cherenkov light is sharp as the muon travels relatively straight as it comes to a stop. Distant hit PMTs come from scattered light and Cherenkov light from delta-rays.

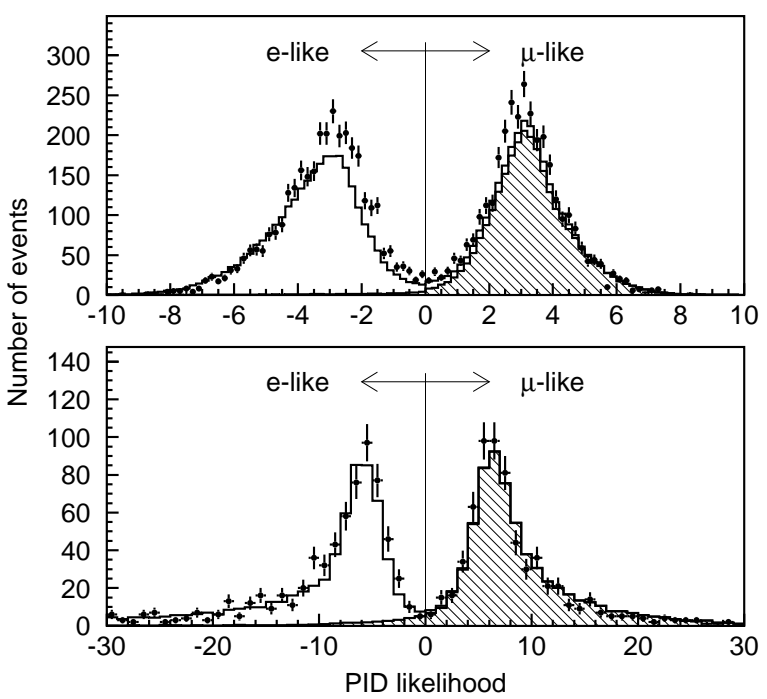

FIG. 22: The distribution of particle identification likelihood for sub-GeV (top) and multi-GeV (bottom) FC single-ring events, comparing SK data (points) and atmospheric neutrino Monte Carlo (histograms). The Monte Carlo includes neutrino oscillation with $\left(\sin ^{2} 2 \theta=1.00, \Delta m^{2}=2.1 \times 10^{-3} \mathrm{eV}^{2}\right)$. The hatched histograms show the $v_{\mu}$ charged current interactions.

analyses, electrons in the time interval $800 \mathrm{~ns}$ to $1200 \mathrm{~ns}$ after the primary trigger are excluded, owing to this splitting effect as well as a reduced efficiency due to electrical reflection on the PMT cables. The contamination level for these criteria is very good, with no events out of 32000 stopping cosmic ray muons having more than one decay electron. The efficiency for fully-contained sub-GeV neutrino interactions was

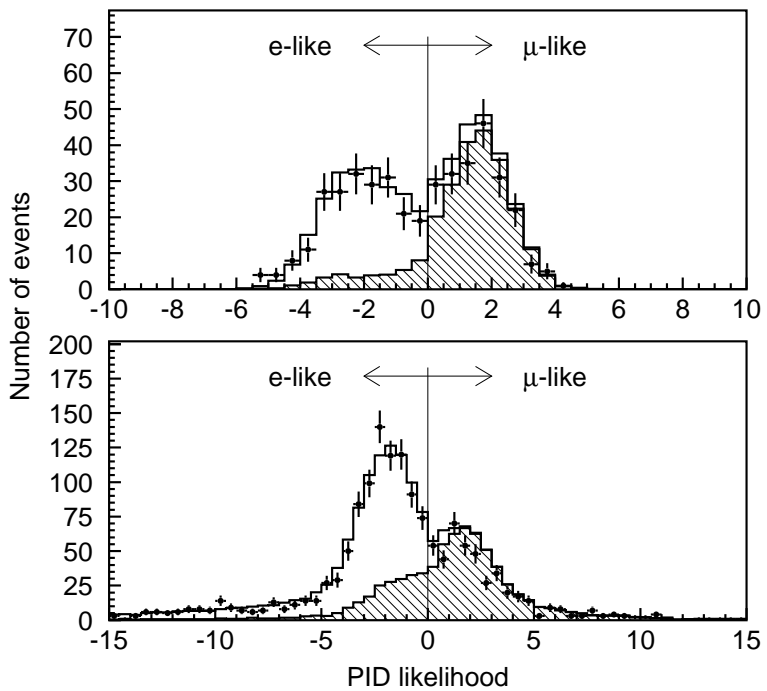

FIG. 23: Particle ID likelihood distribution for sub-GeV (top) and multi-GeV (bottom) FC multi-ring events and the brightest ring of the multi-ring events. Points show the data and the histograms show the Monte Carlo results. The Monte Carlo includes neutrino oscillation with $\left(\sin ^{2} 2 \theta=1.00, \Delta m^{2}=2.1 \times 10^{-3} \mathrm{eV}^{2}\right)$. The hatched histograms show the $v_{\mu}$ charged current interactions.

estimated by Monte Carlo to be $80 \%$ for $\mu^{+}$and $63 \%$ for $\mu^{-}$, where the lower efficiency is due to $\mu^{-}$capture on ${ }^{16} \mathrm{O}$.

\section{Background and Efficiency}

The main sources of the background for the FC sample are cosmic ray muons, neutrons generated by high energy cosmic ray muons and PMT flasher events. The contamination of the background events was estimated for lower energy (visible energy lower than $1.33 \mathrm{GeV}$, called $s u b-\mathrm{GeV}$ ) and higher energy (visible energy higher than $1.33 \mathrm{GeV}$, called multi-GeV) samples separately, since the contamination could have an energy dependence. The cosmic ray muon background contaminations to the final FC sample were estimated to be $0.07 \%$ for sub-GeV and $0.09 \%$ for multi-GeV $\mu$-like events. They were estimated using the distribution of the distance of the vertex position from the inner detector wall along the particle direction. The neutron background contamination was estimated to be $0.1 \%$ for sub-GeV $e$-like events and multi-GeV $e$-like events. This was estimated using the distribution of the distance of the vertex position from the inner detector wall $\left(D_{\text {wall }}\right)$. The contaminations from PMT flasher events were estimated to be $0.42 \%$ for sub-GeV $e$-like events and $0.16 \%$ for multi-GeV $e$-like events using the goodness of the vertex fitting and the $D_{\text {wall }}$ distributions.

The reduction efficiency was estimated using the atmospheric neutrino MC. The detection efficiency for events which satisfy the data reduction conditions (i)-(iv) was $99.15 \%$ for events which have a true vertex in the fiducial volume, $E_{v i s}>30 \mathrm{MeV}$, and less than 10 hits in the largest OD cluster. The systematic error in the event reduction was esti- 
mated to be $0.2 \%$. The main source of the systematic error in the event reduction was the flasher cut based on the pattern matching algorithm. The systematic error for this cut was estimated by mixing the different flasher samples in the atmospheric MC and comparing the reduction efficiencies. The inefficiency was estimated to be $0.7 \% \pm 0.2 \%$, in which $0.2 \%$ was considered as the systematic uncertainty. The systematic errors for other reduction steps were negligibly small because the reduction efficiency was almost $100 \%$ and the distributions of cut parameters for data agree with that of MC. In the early stage of the experiment, an independent data selection and reconstruction program was also employed; the results were compared and event samples and classifications were found to agree by around $95 \%[1]$.

\section{B. Partially-Contained Events}

\section{Data Reduction}

The data reduction for PC events differed from the reduction for FC events because of the presence of additional hits in the OD. Because these extra hits result from the exiting particle (usually a muon), a simple criterion based on the number of hit OD tubes could not be used to reject cosmic ray background. The criteria used to identify partially-contained events are as follows:

(i) Low energy events with fewer than 1000 total p.e.'s in the inner detector were removed, corresponding to muons (electrons) with momentum less than 310 (110) MeV/c. By definition, an exiting particle in the PC sample must have reached the $\mathrm{OD}$ from the inner fiducial volume, and so must have had a minimum track length of about $2.5 \mathrm{~m}$ (corresponding to muons with $\geq 700 \mathrm{MeV} / c$ momentum).

(ii) Events for which the width of the time distribution of hits in the OD exceeded 260 nsec were rejected, as well as events with two or more spatial clusters of OD hits. These cuts eliminated many through-going muons, which typically left two well separated clusters in the OD. Muons which clipped the edges of the detector were eliminated based upon the topology of the OD cluster. Cosmic ray muons which entered and stopped in the inner volume of the detector were eliminated by excluding events with a relatively small number of ID photoelectrons near the OD cluster (1000 p.e.'s within $2 \mathrm{~m}$ ). This cut did not remove PC neutrino events because PC events produced large numbers of photoelectrons (typically 3500 p.e.'s) in the region where the particle exited.

(iii) In the next step, a simple vertex fit and p.e. weighted direction estimation were used. A requirement of $\leq 10$ hits in the OD within $8 \mathrm{~m}$ of the back-projected entrance point was imposed. Also in this step, flasher events were removed by using their broader timing distribution feature.

(iv) The remaining background still had muons which left few or no entrance hits in the OD. These events were rejected by requiring the angle subtended by the earliest inner detector PMT hit, the vertex, and the back-projected entrance point be $>37^{\circ}$. Remaining corner clipping muons were rejected by requiring a fitted vertex at least $1.5 \mathrm{~m}$ away from the corners of the ID volume. A through-going muon fitter was also applied to reject events with a well fitted muon track greater than $30 \mathrm{~m}$ long.

(v) In the last reduction step, various remaining background events were eliminated by several selection criteria: (1) Fullycontained events were eliminated by requiring that $\mathrm{PC}$ events have more than 9 hits in the most highly charged cluster in OD; (2) A minimum requirement of 3000 total p.e.'s in the inner detector, which corresponds to $350 \mathrm{MeV}$ of visible energy, well below that of any exiting muon, was applied to get rid of low energy background events; (3) Clusters in the OD were searched for again with the same clustering algorithm used in the 2 nd reduction step but with different clustering parameters. Events were eliminated if there existed two or more clusters with more than 10 p.e.'s and they were apart by more than 20 meters. Some obvious through-going muons were removed by this cut; (4) After those steps, most remaining background events are due to the imprecision of the fast fitters used to quickly filter the data stream. A precise fitting algorithm was then applied to obtain more accurate information on ring direction and vertex position. With much more accurate information of the event, we were able to eliminate most remaining through-going and stopping events based on their distinct geometry and OD signatures. (5) Some through-going muons have a very special geometry-they passed through the tank vertically along the wall of ID. These events were eliminated by counting the number of p.e.'s and hits in the OD within the region defined by an $8 \mathrm{~m}$ sphere around the top and bottom fringes and checking the time interval between the average timings of those top and bottom hits. (6) Remaining cosmic ray muon background events are those entering the ID through relatively weak OD regions-there are four holes covered by veto counters on the top of OD through which cables run. Events with a veto counter hit were eliminated, as well as those satisfying a detailed cosmic ray muon consistency requirement.

(vi) After this final reduction step, events were scanned by physicists to check the data quality. However, no event was rejected based on the scanning. Finally, the vertex was required to be within a fiducial volume, 2 meters from the wall of the inner detector. The final event sample is an almost $100 \%$ pure $v$ sample. The background contamination has been estimated to be about $0.2 \%$.

Table 11 shows the number of events after each reduction step and the detection efficiency of PC events as a function of reduction steps.

\section{Event Reconstruction}

The partially-contained events were reconstructed using inner detector PMT information by similar vertex, direction fit and ring-counting algorithms, as were applied to fully contained events.

For some PC events, however, the direction fit was slightly modified. Under some conditions, rather than using the results of the precise fitting algorithm for the PC event direction, outer detector spatial information was used instead. In order 


\begin{tabular}{l|c|c}
\hline \hline Reduction step & Data & Monte Carlo \\
\hline \hline Trigger & 1889599293 & $1,417.0(100.0 \%)$ \\
First reduction & 34536269 & $1,402.8(99.0 \%)$ \\
Second reduction & 5257443 & $1,334.7(94.2 \%)$ \\
Third reduction & 380053 & $1,318.7(93.1 \%)$ \\
Fourth reduction & 53825 & $1,246.2(87.9 \%)$ \\
Fifth reduction & 1483 & $1,201.0(84.8 \%)$ \\
Fiducial volume & 911 & $1,129.6(79.7 \%)$ \\
\hline \hline
\end{tabular}

TABLE II: Number of events after each reduction step for partiallycontained events during 1489 days of the detector live-time. The Monte Carlo efficiencies are for events whose real vertices are in the fiducial volume and the number of outer detector hits more than 9 . In the last line, we used the events whose fitted vertices are inside the fiducial volume both for data and Monte Carlo. The Monte Carlo does not include neutrino oscillation.
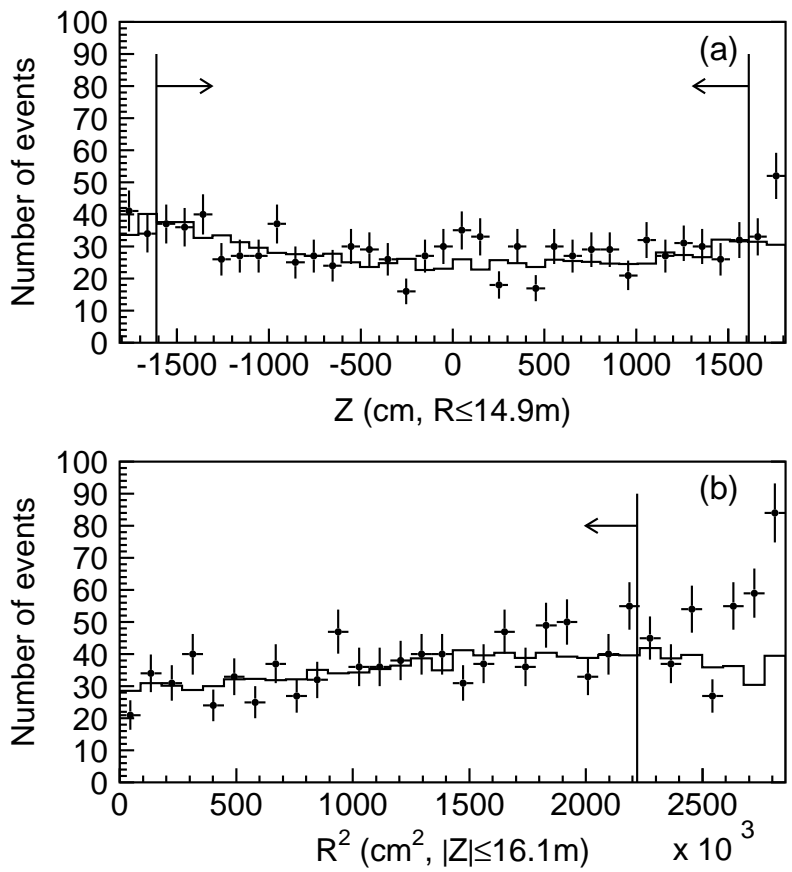

FIG. 24: The distribution of partially-contained event vertices in the (a) $z$-coordinate and (b) $r^{2}$-coordinate, comparing SK data and atmospheric neutrino Monte Carlo. The Monte Carlo includes neutrino oscillation with $\left(\sin ^{2} 2 \theta=1.00, \Delta m^{2}=2.1 \times 10^{-3} \mathrm{eV}^{2}\right)$.

to use the outer detector cluster for the direction, the number of tubes in the largest OD cluster was required to be greater or equal to 20. If this condition was satisfied, and also, if the ID PMT nearest to the projected ID exit point of the fitted track with more than 200 p.e.'s was more than $200 \mathrm{~cm}$ away (i.e. no clear ID exit point), or if there was a clear exit point in the ID but yet there were more than 800 ID PMT hits with more than 200 p.e.'s in each of them (i.e. saturated in our electronics), then the vector from the fitted vertex to the largest OD cluster was used for the PC event direction. Otherwise, the standard direction provided by the precise fitting algorithm was used.
The estimated vertex position resolution for PC events was $64 \mathrm{~cm}$. The angular resolution for the penetrating particle in a PC event was estimated to be $2.8^{\circ}$.

Finally, the fiducial volume cut was applied. The event rate in the fiducial volume was 0.62 events/day.

\section{Background and Efficiency}

The background for the PC sample originates from cosmic ray muons. They were efficiently removed by the reduction steps mentioned above. We estimated the contamination of non-neutrino events in the fiducial volume by two methods. One method utilized scanned results. After applying all the reduction steps, all the events were scanned and the estimated fraction of background contamination was found to be $0.2 \%$ in the fiducial volume. Another method was by examining the vertex distribution of non-neutrino events as a function of distance from the wall. By extrapolating the distribution from outside the fiducial volume, we obtained that the contamination of the background was less than $0.1 \%$. Since these two results were statistically consistent, we took the larger number $(0.2 \%)$ as the contamination of background events in the fiducial volume. Figures 24 (a) and (b) show the distribution of the vertex position for both data and $\mathrm{MC}$ as a function of the $z$-coordinate and $r^{2}$-coordinate. Some contamination of background is evident near the side and top PMT walls. However, no evidence for substantial background contamination is seen in the fiducial volume.

The PC reduction efficiency was estimated based on Monte Carlo events. The definition of partially-contained events is that (1) the interaction point of the parent neutrino is inside the fiducial volume, and (2) number of outer detector hits within $8 \mathrm{~m}$ around an estimated exiting point is larger than nine. We applied those five reduction steps to the atmospheric neutrino Monte Carlo sample and after each reduction step, we counted the number of PC events left inside fiducial volume and calculated the efficiency of this particular reduction step. We found the overall the efficiency of the reduction for PC neutrino events was $79.7 \%$.

The systematic uncertainties on the reduction efficiency from the first to the fourth steps were estimated by two methods. For the ID contribution, they were estimated by comparing the distributions of cut parameters used in the reduction criteria for the data and Monte Carlo. For the OD part, we created Monte Carlo samples with two different sets of ODrelated tuning parameters. Then, the change in reduction efficiency is the OD-related systematic error. The two sets produced different amounts of light in the OD within the limits of good overall agreement with the standard tuning sample (several hundreds of well-measured stopping muons). The estimated uncertainties were $1.5 \%$ and $1.4 \%$ for inner- and outer-detector related selection criteria, respectively.

The systematic uncertainty in the fifth reduction efficiency is mainly from ID variables and involves the precise fitting algorithm. The main contributions come from the cluster cut mentioned above. The uncertainty on the cluster cut was estimated by examining the distributions of cut variables. The 
uncertainties in other cuts were assumed to be the inefficiency in each cut, since the inefficiency itself was small compared with other errors. Combining these estimated uncertainties, we obtained the value of $1.6 \%$ for the fifth reduction step.

Thus the overall systematic uncertainty in the reduction of the PC events was estimated to be $2.6 \%$.

\section{Upward-going muon Data}

\section{Data Reduction}

The upward-going muons observed in Super-K are classified into two categories: (1) upward stopping muon events having only an entrance signal in the OD; and (2) upward through-going muon events having both entrance and exit signals in the OD. The criteria used in Sec.VB 1 to determine an event entry or exit were used: 10 OD hits in-time and within $8 \mathrm{~m}$ of the tracks projected entry or exit point constitutes a muon entry or exit signal. We required the geometrical trajectory of through-going muons to be greater than $7 \mathrm{~m}$ in the inner detector, and we imposed an equivalent $7 \mathrm{~m}$ path-length cut on upward stopping muons based on the momentum reconstruction using Cherenkov light.

The purpose of the data reduction is to isolate the upward muon events and the horizontal muon events (needed for background estimation), provide a classification of stopping or through-going muon type, and to reject the background from cosmic ray muons and noise such as flashing PMTs. Decay electrons associated with stopping muons were also saved. A charge cut of $8,000 \leq Q<1,750,000$ p.e.'s in the ID was applied. For a muon, $Q($ p.e. $) \simeq 25 \cdot L(\mathrm{~cm})$, ensuring that that we can detect all muons with path-length $\geq 7 \mathrm{~m}$ while eliminating events at lower energies. At very high ID charge corresponding to $\simeq 1,750,000$ p.e.'s the ID electronics becomes saturated causing the muon fitters fail.

To isolate the rate of about one neutrino induced upwardgoing muon per day from the remaining background of about $2 \times 10^{5}$ cosmic ray muons, we used a logic tree involving as many as seven different muon fitters. Some of these fitters were specialized to fit stopping muons, others were specialized for through-going muon events, and some of them were specialized to fit muon events with Bremsstrahlung. The main logic behind the upward-going muon reduction was that if a muon fitter classifies an event as upward with a goodness of fit which was above the fitter's goodness threshold then the event was automatically saved. Conversely, if a muon fitter classified an event as downward with a goodness of fit which was above the threshold then the event was automatically rejected. If a muon fitter classified an event as horizontal and with goodness above threshold, or if the fitter could not give a good fit for the event, the event was passed to the next fitter. This sequence continued until all the events had passed through all the fitters or had been classified. If no fitter was able to give a good fit then this event was automatically rejected. If at least one fitter classified this event as horizontal then the event was saved. All events from the output of the upward muon reduction were then passed to the precise fitter which is described in Sec. $\mathrm{VC} 2$

\section{Event Reconstruction}

All events from the output of the upward-going muon reduction were passed through the precise fitter. The basic algorithm was identical to that used for the vertex and direction determination for single ring fully-contained and partiallycontained events. The fitter assumes that the particle is a muon and the vertex position of the event is at the inner detector surface. However, when the muon produces an energetic electro-magnetic shower, the assumption of single nonshowering muon does not give an accurate direction. For these events, the information of OD hit is used to determine the particle direction. The angular resolution of the fitter was about $1.0^{\circ}$ for both through-going and stopping muons. Taking into account multiple scattering from the point of muon creation, $68 \%$ of through-going and stopping muons fit within $1.3^{\circ}$ and $2.4^{\circ}$ of the muon's true initial direction, respectively. The direction determined by this fitter was used in the neutrino oscillation analysis.

\section{Background and Efficiency}

The effective detection efficiency for the data reduction process was estimated by a Monte Carlo simulation, and was found to be $102.4 \%$ for upward stopping muons and $95.9 \%$ for upward through-going muons. The efficiency higher than $100 \%$ for upward stopping muons is due to a slight bias in the separation of stopping and through-going muons, causing a small fraction of the more numerous through-going muons to be misidentified as stopping muons. The up/down symmetry of the detector geometry allows a check of this Monte Carlo efficiency calculation using real cosmic ray induced downward-going muons. The efficiency is approximately constant for $-1<\cos \Theta<0$; bin-by-bin efficiencies are listed in Table XII in the Appendix.

After the reduction, the precise fitter described in Sec.VC2 was applied to determine the entry position and the muon direction. This is the final direction used for further physics analysis, including the determination of upward versus downward classification. Events selected as upward by this direction were then scanned by eye with a graphical event display program in order to reject difficult-to-remove instances of corner clipping or bremsstrahlung cosmic ray muons and noise events. The event scanning only rejected events judged to be background and did not change the direction and the vertex decided by the precise fitter, nor the stop/through judgment made by the reduction programs using the entrance and exit points of this fit. The event scanning was done independently by two physicists and testing had shown that both scanners had never rejected the same good upward-going muon event. About $50 \%$ of the events remaining after all automated reduction steps were rejected by this final scan. Table [II summarizes the data reduction for upward muons. 


\begin{tabular}{|c|c|c|c|c|}
\hline \multirow[t]{2}{*}{ Reduction step } & \multicolumn{2}{|c|}{ Data } & \multicolumn{2}{|c|}{ Monte Carlo } \\
\hline & stopping & through-going & stopping & through-going \\
\hline Trigger & \multicolumn{2}{|c|}{2129729843} & $697.1(100 \%)$ & $1741.0(100 \%)$ \\
\hline Reduction & \multicolumn{2}{|c|}{89911} & $693.9(99.5 \%)$ & $1722.3(98.9 \%)$ \\
\hline Precise fitter $(\cos \theta \leq 0)$ & \multicolumn{2}{|c|}{4266} & $692.4(99.3 \%)$ & $1721.7(98.9 \%)$ \\
\hline Scan & \multicolumn{2}{|c|}{2447} & - & - \\
\hline Stop-through separation and $E_{\mu} \geq 1.6 \mathrm{GeV}$ & 458 & 1856 & $713.5(102.4 \%)$ & $1669.5(95.9 \%)$ \\
\hline CR BG subtraction (subtracted ev) & $417.7(40.3)$ & $1841.6(14.4)$ & - & - \\
\hline
\end{tabular}

TABLE III: Number of events after each step of the data selection for upward muons during 1646 days of the detector live-time. The Monte Carlo efficiencies are relative to the generated events with track length longer than $7 \mathrm{~m}$ (for through-going muons) or with energy higher than $1.6 \mathrm{GeV} / c$ (for stopping muons). The efficiencies are for Monte Carlo upward muon events with track length in the inner detector longer than $7 \mathrm{~m}$ (for through-going muons) or with muon momentum at the wall of the inner detector higher than $1.6 \mathrm{GeV} / \mathrm{c}$ (for stopping muons). The Monte Carlo does not include neutrino oscillation.

Near the horizon, horizontal cosmic ray muons are a nonnegligible source of background for both through-going and stopping upward muons. Because of finite fitter resolution and multiple Coulomb scattering of muons in the nearby rock, some downward going cosmic ray muons may appear to be coming from $\cos \Theta<0$. Figure 25 shows the zenith versus azimuth directions for the upward-going muon sample. Clusters of cosmic ray downward muons are seen for relatively thin overburden directions. Figure 26 shows the zenith angle distribution of upward muon candidates near the horizon for two different regions in azimuth. The thick overburden region has negligible downward going cosmic ray muon contamination, even above the horizon. The thin overburden region has non-negligible contamination. The shape of the distribution above the horizon was extrapolated below the horizon to estimate the background contamination in the upward muon sample. The number of background events, based on Fig.26 to the upward stopping muon signal were estimated to be $14.4 \pm 7.2$ (stat) \pm 6.0 (sys) and $40.3 \pm 13.7$ (stat) $\pm 4.3($ sys $)$ events for the through-going and stopping muon samples, respectively. Horizontal muon background was contained in the $-0.1<\cos \Theta<0$ zenith angle bin, and was subtracted from this bin. The stopping muon contamination is larger than the through-going contamination since lower muon energies allow larger scattering angles.

Away from the horizon, a potential source of upward particles is the photo-production of pions by energetic muons that pass nearby the detector [78]. The pathlength requirement of $7 \mathrm{~m}$ within the inner detector limits the background from this source to $\simeq 0.01 \%$ in the upward through-going muon data sample and $\simeq 0.3 \%$ in the upward stopping muon data sample.

\section{4. $\quad$ Expected Upward-Going Muon Signal}

The expected upward-going muon flux was calculated using the same tools as for the contained vertex events, extended to higher energies and to outside the detector volume. The input neutrino flux (see Sec. III) in Ref. [28] was used up to

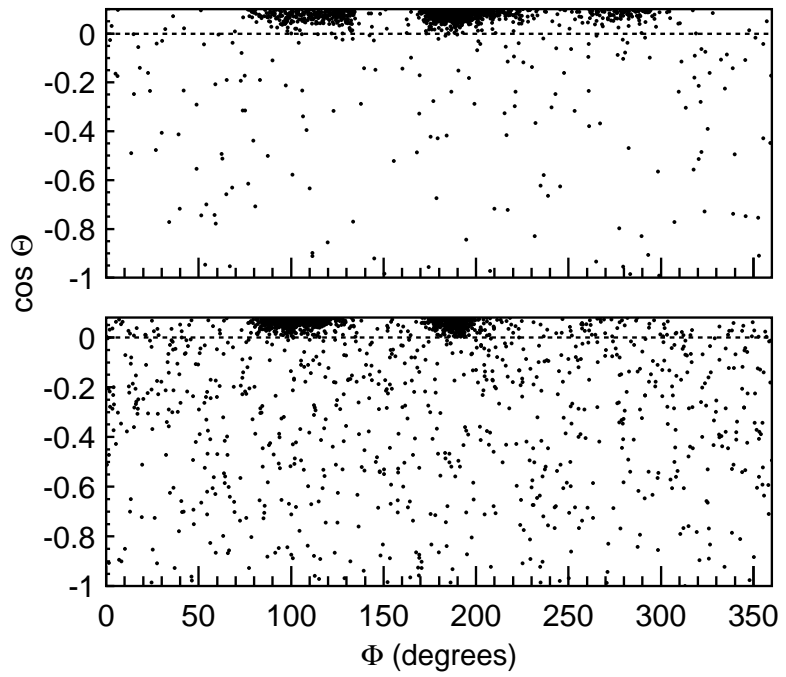

FIG. 25: The zenith versus azimuth directions for a 641.4 day sample of upward muons used to estimate background contamination. The dense regions of events near the horizon correspond to thin regions of the mountainous overburden. The upper panel is for upward stopping muons and the lower panel is for upward through-going muons.

$1 \mathrm{TeV}$. At $1 \mathrm{TeV}$, the calculated flux in Ref. [79] was rescaled to that in Ref. [28]. Above $1 \mathrm{TeV}$, the rescaled flux in [79] was used up to $100 \mathrm{TeV}$. The target volume for these neutrinos is primarily the rock around the detector, parameterized as standard rock, with $\mathrm{Z}=11, \mathrm{~A}=22$ and density $=2.7 \mathrm{gm} / \mathrm{cm}^{3}$. However, neutrinos interacting in the water of the OD and insensitive region can also be seen as upward-going muons ( $1.8 \%$ of through-going and $6.6 \%$ of stopping muons), so water interactions were also simulated.

The neutrino interactions were modeled as discussed in Sec.IV The same GEANT detector simulation discussed previously was used to track muons from the interaction vertex through the rock into the detector itself. The output of the detector simulation was passed through the same reduction and fitting routines as was the real upward-going muon data. 100 


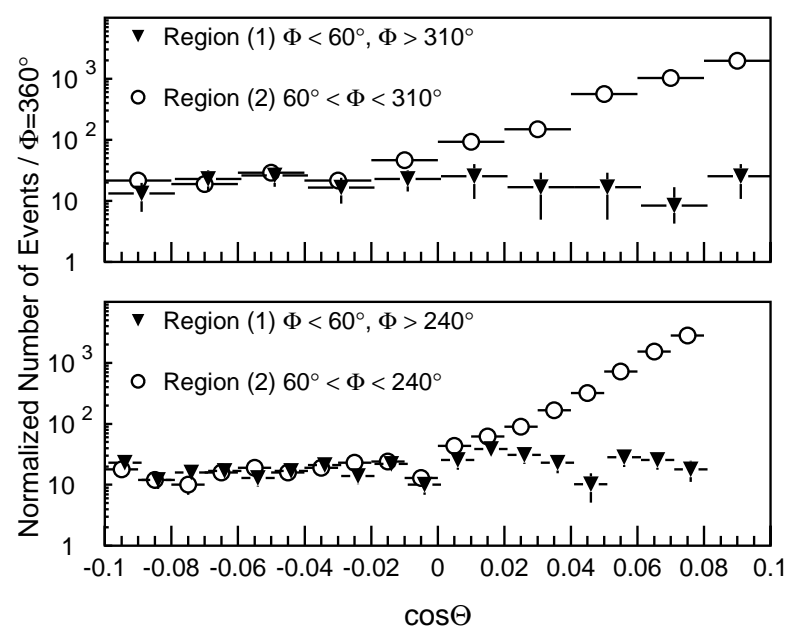

FIG. 26: The zenith angle distribution of upward muon candidates near the horizon for two different regions in azimuth. Regions (1) and (2) are thin and thick overburden respectively. The live-time of upward and downward events is 1645.9 days and 641.4 days respectively. The number of downward events is normalized to be 1645.9 days live-time. The upper panel is for upward stopping muons and the lower panel is for upward through-going muons.

years equivalent exposure was generated. The results of this Monte Carlo data before and after the reduction were used to estimate efficiencies and systematic errors as well as provide an expected upward-going muon signal. Table the detection efficiency at each step of the data reduction.

\section{Observations}

\section{Contained Vertex Events}

We have accumulated 1489.2 days of FC and PC data from May 17, 1996 to July 16, 2001. These are compared with statistically larger samples of Monte Carlo events based on the two neutrino interaction models [47, 48], both equivalent to 100 years of the detector exposure. However, upward-going Monte Carlo muons were only generated based on NEUT. Both Monte Carlo samples were generated based on the flux model of Ref. [28]. The Monte Carlo samples were processed by the same event-selection and event reconstruction steps as the real events. Throughout this paper, unless otherwise indicated, we used Monte Carlo events generated based on the flux model of Ref [28] and the NEUT neutrino interaction model [47].

Fully-contained events were divided into two sub-samples according to the reconstructed visible energy. We refer to the event sample below $1.33 \mathrm{GeV}$ as sub-GeV, and above $1.33 \mathrm{GeV}$ as multi-GeV. Fully-contained events were further divided into the events with single reconstructed Cherenkov ring, single-ring, and events with more than one rings, multiring. All single-ring events were classified as either $e$-like or $\mu$-like based on the PID result. Lower energy cuts were applied only to the single-ring sample, $P_{e}>100 \mathrm{MeV} / \mathrm{c}$ for $e$-like and $P_{\mu}>200 \mathrm{MeV} / \mathrm{c}$ for $\mu$-like. In addition, multiring events were used to study the atmospheric neutrino flux. A simple set of criteria that the most energetic ring in a multi-ring event was $\mu$-like with $P_{\mu}>600 \mathrm{MeV} / c$ and $E_{v i s}>$ $600 \mathrm{MeV}$ selected relatively pure $\mathrm{CC} v_{\mu}$ events. The estimated fraction of $\mathrm{CC}_{\mu}$ in this sample was $90.5 \%$ and $94.9 \%$ for the sub- and multi-GeV energy ranges, respectively. On the other hand, a similar set of criteria for $e$-like events obtained only a $54.4 \%$ pure $\mathrm{CC}_{e}$ sample, and therefore we decided to use multi-ring $\mu$-like events only.

Table IV summarizes the number of observed events in the sub-GeV and multi-GeV samples as well as the expected number of events in the absence of neutrino oscillations. The fraction of various neutrino interaction modes, predicted by the Monte Carlo sample, are also listed. Figure 27 shows the event rates for contained events as a function of the elapsed days. The event rate should change due to the solar modulation. However, the expected decrease in the event rate from minimum solar activity (which approximately corresponds to the period when the SK-I started taking data) to maximum solar activity (which approximately corresponds to the period when the SK-I finished taking data) period is 6-7\% for sub$\mathrm{GeV}$ events and 3-4\% for multi-GeV events. The data cannot distinguish a constant event rate from the expected rate change due to solar activity.

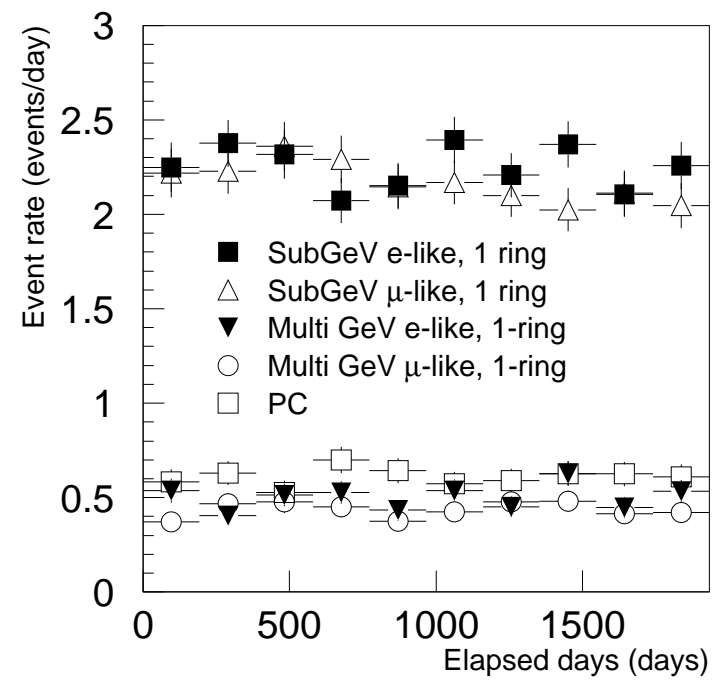

FIG. 27: Event rates as a function of elapsed SK running time for contained events.

Figures 17 24 show the reconstructed vertex distributions for FC and PC events projected on the $z$ and $r^{2} \equiv\left(x^{2}+y^{2}\right)$ axes. The shape of the vertex distributions of the data and MC agree well in the fiducial volume. Near the fiducial volume boundary, the event rate for FC events slightly decreases, and the event rate for PC events increases. This is because energetic muons produced by neutrino interactions near the wall tend to escape and are identified as PC events. Peaks near 


\begin{tabular}{|c|c|c|c|c|c|c|c|c|}
\hline & \multicolumn{2}{|c|}{$\begin{array}{r}\text { Data Monte Carlo } \\
(\mathrm{NEUT}) \\
(\text { Flux A })\end{array}$} & $\mathrm{CC} v_{e}$ & $\mathrm{CC} v_{\mu}$ & $\mathrm{NC}$ & \begin{tabular}{|l} 
(NEUT) \\
(Flux B)
\end{tabular} & $\begin{array}{r}\text { Monte Ca } \\
(\mathrm{NEUT}) \\
(\text { Flux C) }\end{array}$ & $\begin{array}{l}\text { lo } \\
\text { (NUANCE) } \\
\text { (Flux A) }\end{array}$ \\
\hline sub-GeV & 8941 & 9884.3 & & & & 9967.8 & 10619.4 & 9074.2 \\
\hline single-ring & 6580 & 7092.6 & & & & 7273.2 & 7643.3 & 6694.0 \\
\hline$e$-like & 3353 & 2879.8 & $2533.9(88.0 \%)$ & $66.3(2.3 \%)$ & $279.6(9.7 \%)$ & 2944.2 & 3069.5 & 2762.3 \\
\hline$\mu$-like & 3227 & 4212.8 & $22.8(0.5 \%)$ & $3979.7(94.5 \%)$ & $210.3(5.0 \%)$ & 4329.0 & 4573.9 & 3931.6 \\
\hline multi-ring & 2361 & 2791.7 & & & & 2694.6 & 2976.0 & 2380.2 \\
\hline$\mu$-like & 208 & 322.6 & $11.6(3.6 \%)$ & $292.0(90.5 \%)$ & $18.9(5.9 \%)$ & 301.5 & 342.1 & 274.0 \\
\hline \multicolumn{6}{|c|}{$R=0.658 \pm 0.016($ stat $) \pm 0.035($ sys $)$} & 0.655 & 0.646 & 0.676 \\
\hline multi-GeV & 2901 & 3472.0 & & & & 3212.6 & 3708.7 & 3179.3 \\
\hline single-ring & 1397 & 1580.4 & & & & 1456.8 & 1676.6 & 1463.7 \\
\hline$e$-like & 746 & 680.5 & $562.2(82.6 \%)$ & $47.6(7.0 \%)$ & $70.7(10.4 \%)$ & 635.3 & 729.2 & 635.3 \\
\hline$\mu$-like & 651 & 899.9 & $3.6(0.4 \%)$ & $894.2(99.4 \%)$ & $2.1(0.2 \%)$ & 821.4 & 947.4 & 828.4 \\
\hline multi-ring & 1504 & 1891.6 & & & & 1755.9 & 2032.1 & 1715.5 \\
\hline$\mu$-like & 439 & 711.9 & $16.6(2.3 \%)$ & $675.8(95.0 \%)$ & $19.4(2.7 \%)$ & 645.9 & 749.1 & 618.9 \\
\hline partially-contained & 911 & 1129.6 & $20.8(1.8 \%)$ & $1098.8(97.3 \%)$ & $10.0(0.9 \%)$ & 1065.0 & 1236.6 & 1074.9 \\
\hline \multicolumn{6}{|c|}{$R_{F C+P C}=0.702_{-0.030}^{+0.032}($ stat $) \pm 0.101$ (sys) } & 0.705 & 0.699 & 0.699 \\
\hline
\end{tabular}

TABLE IV: Summary of the sub-GeV, multi-GeV and PC event samples compared with the Monte Carlo prediction based on the neutrino interaction model of Ref. [47] (NEUT) and neutrino flux calculation of Ref. [28], as well as different flux models. Fluxes A, B and C refers to [28], [25] and [29], respectively. The Monte Carlo prediction with NUANCE [48] and Flux A is also shown. The Monte Carlo predictions do not include neutrino oscillations.

the edge of the ID for both the MC and the data are caused by a constraint of the vertex reconstruction programs: the reconstruction of the vertex is restricted within the ID, and the events whose vertex is estimated outside of the ID are constrained to be within the ID, where they pile up at the edge. The peak at $z=1810.0 \mathrm{~cm}$ in the distribution for FC data is caused by the cosmic ray muons passing through inefficient regions of the OD. These muons are safely rejected by the fiducial volume cut.

Fig. 28 shows the reconstructed momentum distributions for FC single-ring events. The data and MC show good agreement except for significantly fewer numbers of FC $\mu$ like events. Fig. 29 shows the visible energy distribution for FC multi-ring $\mu$-like and PC events. The PC data have more events than the Monte Carlo prediction at the highest energies. This could indicate that the neutrino energy spectrum in the Monte Carlo is too soft around $100 \mathrm{GeV}$. We note that the upward through-going data also suggest that the neutrino energy spectrum is too soft (see Table $\nabla \mathbf{~ I}$ ) The neutrino energy spectrum up to $1 \mathrm{TeV}$ was considered in the Monte Carlo prediction for the FC and PC samples. We estimated that two PC events are expected with visible energy above $100 \mathrm{GeV}$ from neutrinos above $1 \mathrm{TeV}$.

The flavor ratio of the atmospheric neutrino flux, $\left(v_{\mu}+\right.$ $\left.\bar{v}_{\mu}\right) /\left(v_{e}+\bar{v}_{e}\right)$, is predicted with $3 \%$ accuracy. As shown in Table IV the particle identification for FC single-ring events gives a good estimation of the flavor of the parent neutrinos, and the ratio of the number of $e$-like events and $\mu$-like events, $(\mu / e)$, gives a good estimation of the flavor ratio of the atmospheric neutrinos. We define a $(\mu / e)$ double ra- tio, $R \equiv(\mu / e)_{D A T A} /(\mu / e)_{M C}$. Without neutrino oscillation, $R$ should be consistent with unity. $R$ is measured to be:

$$
R_{\text {sub-GeV }}=0.658 \pm 0.016 \pm 0.035,
$$

for the sub-GeV sample.

A substantial fraction of muons in the multi-GeV energy range exit from the inner detector and are detected as PC events. The partially-contained event sample is estimated to be $97 \%$ pure $C C v_{\mu}$ interactions, even without requiring any particle identification or ring-number cuts. Therefore, we add FC single-ring and PC event totals when calculating $R$ in the multi-GeV range. We measured $R$ in the multi-GeV energy range to be:

$$
R_{\text {multi-GeV }+P C}=0.702_{-0.030}^{+0.032} \pm 0.101 .
$$

Systematic uncertainties in the double ratio $R$ have been discussed in detail in Refs. [1] and [2]. These errors have been re-evaluated and are estimated to be $5.3 \%$ for sub-GeV and $14.4 \%$ for multi-GeV events. The sources of the systematic uncertainties in $R$ are listed in Table $\mathrm{V}$ which include both theoretical and experimental errors. Among the experimental systematic errors, the separation of single- and multi-ring events is the largest source of the systematic uncertainties. As shown in Fig. 18, the distributions of the likelihood difference between the single-ring and multi-ring assumptions have slight shifts in the peak positions between the data and the Monte Carlo. These differences could cause systematic uncertainties in the number of identified single-ring $e$-like and $\mu$-like events (which are summarized in Table $\mathbf{X}$. Since the 


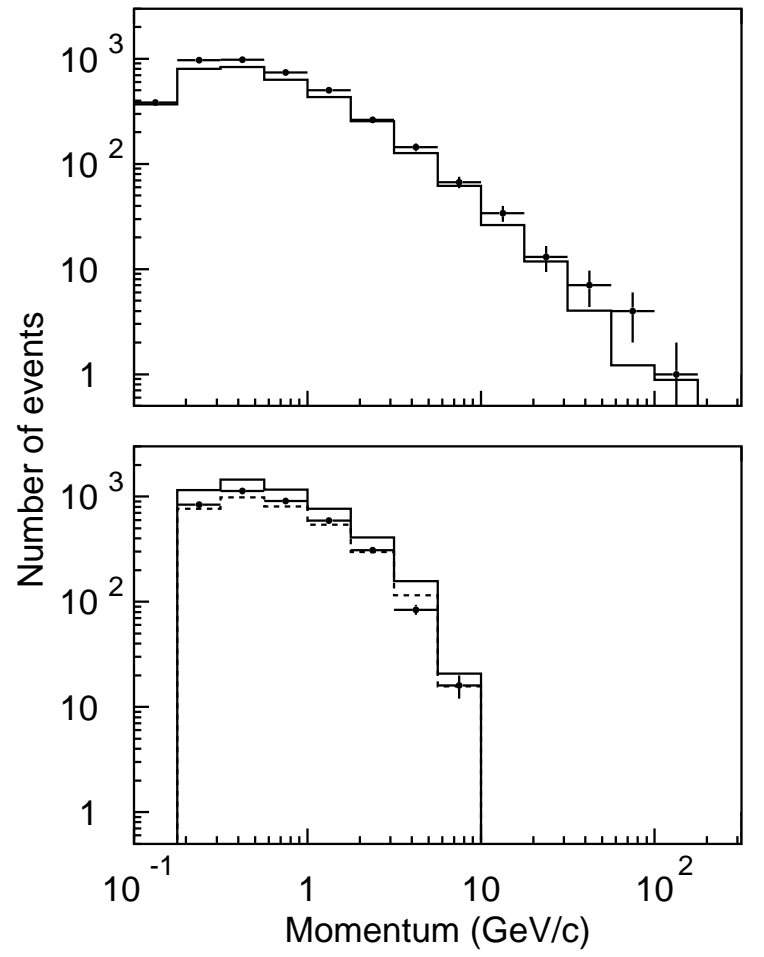

FIG. 28: Momentum distribution of FC single ring $e$-like (top) and $\mu$ like (bottom) events. The sharp cut in the muon momentum spectrum in the high energy end is due to the requirement on fully containment. The points show the data, solid lines show the Monte Carlo prediction without neutrino oscillation and dashed lines show the oscillated Monte Carlo events with $\left(\sin ^{2} 2 \theta=1.00, \Delta m^{2}=2.1 \times 10^{-3} \mathrm{eV}^{2}\right)$.

magnitude of the uncertainty is different between $e$-like and $\mu$ like events, and since the separation of single- and multi-ring is not applied for PC events, the uncertainty in the single- and multi-ring separation causes the uncertainties in the $R$ measurements.

Figure 30 shows the expected $(\mu / e)_{\text {Data }} /(\mu / e)_{M C}$ in the presence of neutrino oscillation for sub- and multi-GeV samples as a function of $\Delta m^{2}$. Data are consistently explained by neutrino oscillations for $\Delta \mathrm{m}^{2}$ in the range of $10^{-3}$ to $10^{-2} \mathrm{eV}^{2}$.

Figure 31 shows the measured and expected numbers of FC and PC events as a function of the cosine of the zenith angle $(\cos \Theta), \cos \Theta=-1$ refers to upward-going and $\cos \Theta=1$ refers to downward-going. Single-ring sub-GeV events are separately shown in two parts, $p_{l} \leq 400 \mathrm{MeV} / c$ and $p_{l}>400$ $\mathrm{MeV} / c$, where $p_{l}$ is the lepton momentum. In the momentum range below $400 \mathrm{MeV} / c$, the angular correlation between the neutrino and outgoing lepton is very poor, the shape of the atmospheric neutrino flux is largely washed out, and the zenith angle distributions for the charged leptons should be approximately flat. Fig. 32 shows the angular resolution of the neutrino directions as a function of the momentum. The angular resolution is defined as the angular difference between the parent neutrinos and the reconstructed directions in which $68 \%$ of the events are included.

We have also studied the azimuthal dependence of the atmospheric neutrino data. This is a sensible consistency check,

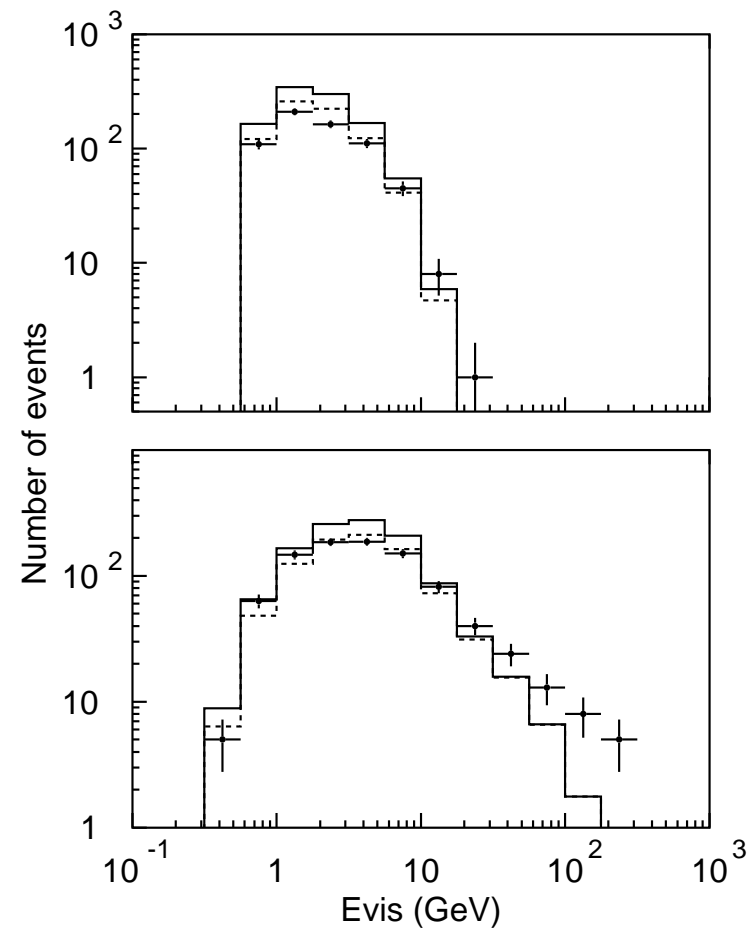

FIG. 29: Visible energy distribution of multi-ring $\mu$-like events (top) and PC events (bottom). The points show the data, solid lines show the Monte Carlo prediction without neutrino oscillation and dashed lines show the oscillated Monte Carlo events with $\left(\sin ^{2} 2 \theta=1.00\right.$, $\Delta m^{2}=2.1 \times 10^{-3} \mathrm{eV}^{2}$ ).

as neutrino oscillation should not cause any azimuthally dependent deficit since all path lengths at a given zenith angle are equal. In a well-selected data sample, the azimuth rates exhibit the famous east-west effect, which was used in the 1930's to demonstrate that cosmic rays were positively charged. The effect is caused by the deflection of primary protons in the earth's magnetic field, where trajectories from the east are blocked by the bulk of the earth. This also results in a deficit of atmospheric neutrinos arriving from the east, with the strongest effect at the lowest neutrino energy. We maximized our sensitivity to this effect by selecting lepton momenta between 400 and $3000 \mathrm{MeV} / \mathrm{c}$ in the zenith angle range $|\cos \Theta|<0.5$. The low momentum cut ensures good pointing resolution, and the high momentum cut diminishes the contribution from high energy primary protons that are insufficiently deflected. The zenith requirement enhances the statistical sensitivity as the depletion only occurs near the horizon. Figure 33 updates our previous result[80] to the final data reported here. That our data reproduces the prediction for this effect implies that the model for geomagnetic cutoff in the flux prediction is accurately accounted for, and checks the basic features of neutrino production and scattering.

While the measured shape of zenith angle distributions for $e$-like events is consistent with expectations, both the FC $\mu$-like and PC samples exhibit significant zenith-angle dependent deficits. The up-down ratio, where $U$ is the number of upward-going events $(-1<\cos \Theta<-0.2)$ and $D$ is the number of downward-going events $(0.2<\cos \Theta<1)$, 


\begin{tabular}{|c|c|c|}
\hline & sub-GeV(\%) & multi-GeV(\%) \\
\hline Prediction of $v_{\mu} / v_{e}$ ratio & 2.7 & 2.2 \\
\hline Prediction of $v / \bar{v}$ ratio & 1.6 & 0.8 \\
\hline$K / \pi$ ratio & 0.6 & 1.9 \\
\hline$E_{V}$ spectral index & 0.6 & 2.3 \\
\hline Sample-by-sample ${ }^{a}$ & - & 2.9 \\
\hline \multicolumn{3}{|l|}{$v$ interaction } \\
\hline quasi-elastic scattering & 1.4 & 1.0 \\
\hline single-meson production & $<0.1$ & 0.3 \\
\hline deep-inelastic scattering & 0.2 & 0.5 \\
\hline coherent-pion production & 0.4 & 0.2 \\
\hline $\mathrm{NC} / \mathrm{CC}$ ratio & 0.5 & 2.0 \\
\hline nuclear effects ${ }^{b}$ & 1.3 & 0.8 \\
\hline Hadron simulation & 0.7 & $<0.1$ \\
\hline FC reduction & 0.1 & 0.1 \\
\hline $\mathrm{PC}$ reduction & - & 1.5 \\
\hline Non- $v$ background & $<0.5$ & $<0.3$ \\
\hline$\mu / e$ separation & 1.3 & 0.6 \\
\hline Single-ring/multi-ring separation & 3.2 & 13.2 \\
\hline Energy calibration & 0.6 & 1.2 \\
\hline MC statistics & 0.5 & 0.9 \\
\hline Total & 5.3 & 14.4 \\
\hline
\end{tabular}

${ }^{a}$ Different flux calculations predict different energy dependences that cannot be explained by a simple spectral index uncertainty. See lower part of Fig. 2 Uncertainty of the relative normalization of the fully-contained multi-GeV and partially-contained sample gives the systematic error in double ratio $R$.

${ }^{b}$ The mean free path of hadrons in ${ }^{16} O$ was changed by $30 \%$. Also the uncertainty in the pion energy spectrum produced by neutrino interactions, defined to be the difference between the interaction models $\mathrm{A}$ and $\mathrm{B}$, is taken into account.

TABLE V: Sources of the systematic errors in double ratio $R(\equiv$ $\left.(\mu / e)_{D A T A} /(\mu / e)_{M C}\right)$ for the sub-GeV and multi-GeV samples. Estimated uncertainty in each source of the systematic error is described in Tables VII VIII IX and X

is measured to be: $U / D=1.133_{-0.059}^{+0.062} \pm 0.009$ for singlering sub-GeV $e$-like events in the momentum range below $400 \mathrm{MeV} / c, U / D=1.082_{-0.060}^{+0.063} \pm 0.024$ above $400 \mathrm{MeV} / c$, $U / D=0.964_{-0.058}^{+0.062} \pm 0.008$ for single-ring sub-GeV $\mu$-like events below $400 \mathrm{MeV} / c, U / D=0.670_{-0.034}^{+0.035} \pm 0.012$ above $400 \mathrm{MeV} / c, U / D=0.961_{-0.079}^{+0.086} \pm 0.016$ for the multi-GeV $e$ like events and $U / D=0.551_{-0.033}^{+0.035} \pm 0.004$ for the single-ring multi-GeV $\mu$-like plus PC events.

Many systematic uncertainties are canceled for the updown ratio and the remaining sources of the uncertainties are: uncertainty in the flux calculation, $0.5 \%$ and $0.8 \%$ for sub$\mathrm{GeV} e$-like and $\mu$-like events in the momentum range below $400 \mathrm{MeV} / c, 2.1 \%$ and $1.8 \%$ for $e$-like and $\mu$-like events above $400 \mathrm{MeV} / c$, and $1.5 \%$ and $0.6 \%$ for multi-GeV $e$-like events and multi-GeV $\mu$-like events plus PC events; uncertainty in the angular dependence of absolute energy calibration, $0.5 \%$ and $0.2 \%$ for sub-GeV $e$-like and $\mu$-like events in the momentum range below $400 \mathrm{MeV} / c, 0.4 \%$ and $0.4 \%$ for $e$-like and $\mu$-like events above $400 \mathrm{MeV} / c$, and $0.8 \%$ and $0.4 \%$ for multi-GeV

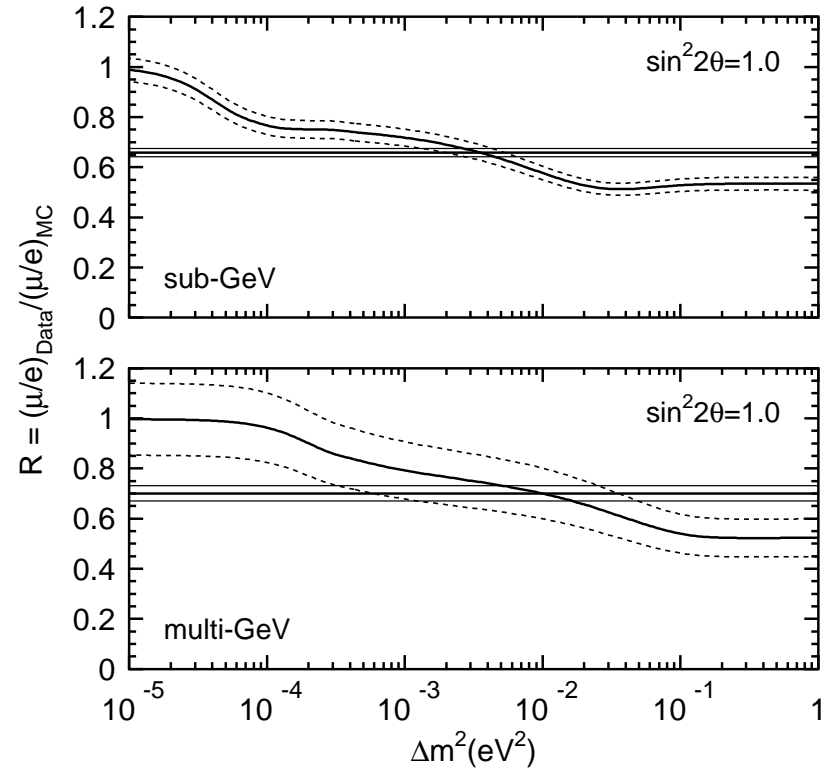

FIG. 30: Expected $(\mu / e)_{\text {Data }} /(\mu / e)_{M C}$ for singe-ring sub- and multi$\mathrm{GeV}+\mathrm{PC}$ samples as a function of $\Delta m^{2}$ for full $v_{\mu} \leftrightarrow v_{\tau}$ mixing. The values for the data together with $\pm 1 \sigma$ statistical errors are shown by the horizontal lines. The systematic errors are shown by the band in the expectation.

$e$-like events and multi-GeV $\mu$-like events plus PC events; and uncertainty in the non-neutrino background such as cosmic ray muons, $<0.4 \%,<0.1 \%,<0.2 \%,<0.2 \%$ for sub-GeV $e$-like, $\mu$-like, multi-GeV $e$-like, $\mu$-like plus PC events, respectively. In total, the systematic uncertainty for $U / D$ is $0.8 \%$ and $0.8 \%$ for sub-GeV $e$-like and $\mu$-like events in the momentum range below $400 \mathrm{MeV} / c, 2.2 \%$ and $1.8 \%$ above 400 $\mathrm{MeV} / c, 1.7 \%$ and $0.7 \%$ for multi-GeV $e$-like and $\mu$-like plus PC events, respectively. While the ratio for $e$-like events is consistent with 1 , the $\mu$-like up-down ratio for the multi-GeV data differs from 1 by more than 12 standard deviations. Figure 34 shows the expected $U / D$ ratios as a function of $\Delta m^{2}$. Data are consistently explained by neutrino oscillations with $\Delta m^{2}$ in the range of $10^{-3.5}$ to $10^{-2} \mathrm{eV}^{2}$.

\section{Upward Muon Events}

The upward-going muon data used in this analysis were taken from May 1996 to July 2001. The detector live-time was 1645.9 days. Though spanning the same period of calendar time, this live-time was larger than that of the contained vertex events because the reconstruction of long path length muons is less sensitive to detector conditions, allowing looser run selection criteria. Fig. 35 shows the event rates as a function of the elapsed days for upward going muons. The event rates for these samples are stable. Table $\nabla \mathbf{~ s u m m a r i z e s ~ t h e ~}$ number of observed events in the upward-going muon data sample and the corresponding flux and expected flux.

The systematic errors on the observed number of events compared to the Monte Carlo predictions are: the $2 \%$ en- 



FIG. 31: The zenith angle distribution for fully-contained 1-ring events, multi-ring events, partially-contained events and upward muons. The points show the data, box histograms show the non-oscillated Monte Carlo events and the lines show the best-fit expectations for $v_{\mu} \leftrightarrow v_{\tau}$ oscillations with $\sin ^{2} 2 \theta=1.00$ and $\Delta m^{2}=2.1 \times 10^{-3} \mathrm{eV}^{2}$. The best-fit expectation is corrected by the 39 systematic error terms, while the correction is not made for the non-oscillated Monte Carlo events. The height of the boxes shows the statistical error of the Monte Carlo.

ergy scale uncertainty leads to $\mathrm{a}_{-1.1}^{+0.9} \%$ error in the stopping muons due to the $1.6 \mathrm{GeV} / c$ cut; the reduction efficiency for stopping (through-going) muons has an uncertainty of ${ }_{-1.25}^{+0.34} \%\left({ }_{-0.54}^{+0.32} \%\right)$; and stopping/through-going separation ${ }_{-0.38}^{+0.29} \%$ (where "+" means through-going muons misidentified as stopping). As in the contained event analysis, comparison of data and expectations is done between observed number of events and the live-time-scaled MC number of events. However, to facilitate comparisons with other exper- iments, these numbers are also presented in units of flux as described in [3, 4]. The additional systematic uncertainty in the observed through-going (stopping) flux comes from effective area of $0.3 \%$ and the live-time calculation $(0.1 \%)$. The absolute expected flux has theoretical uncertainties of at least $20 \%$ in the normalization for high energy $(>100 \mathrm{GeV})$ neutrinos and 5 to $10 \%$ from interaction model differences.

The zenith angle distributions of the upward through-going and stopping muons are shown in Fig. 31 The shape of 


\begin{tabular}{c|c|c|c|c|cc}
\hline \hline event class & $\#$ events & $\begin{array}{c}\text { \# expected } \\
\text { (Flux A })\end{array}$ & $\begin{array}{c}\text { flux } \\
\left(\times 10^{-13} \mathrm{~cm}^{-2} \mathrm{~s}^{-1} \mathrm{sr}^{-1}\right)\end{array}$ & $\begin{array}{c}\text { expected flux } \\
\left(\times 10^{-13} \mathrm{~cm}^{-2} \mathrm{~s}^{-1} \mathrm{~s}^{-1}\right)\end{array}$ & \multicolumn{2}{|c}{ \# expected } \\
(Flux B) & (Flux C) \\
\hline$\Phi_{\text {stop }}$ & 417.7 & 713.5 & $0.381 \pm 0.024_{-0.007}^{+0.005}$ & $0.648 \pm 0.145$ & 681.5 & 790.5 \\
$\Phi_{\text {thru }}$ & 1841.6 & 1669.5 & $1.661 \pm 0.040_{-0.013}^{+0.011}$ & $1.506 \pm 0.337$ & 1644.3 & 1974.9 \\
$\mathcal{R}=\Phi_{\text {stop }} / \Phi_{\text {thru }}$ & 0.227 & 0.427 & $0.229 \pm 0.015 \pm 0.003$ & $0.430 \pm 0.058$ & 0.414 & 0.400 \\
$\Phi_{\text {stop }}+\Phi_{\text {thru }}$ & 2259.3 & 2382.9 & $2.042 \pm 0.046_{-0.015}^{+0.012}$ & $2.154 \pm 0.482$ & 2325.8 & 2765.4 \\
\hline \hline
\end{tabular}

TABLE VI: Summary of observed and expected results for upward-going muons during 1645.9 live-days. The first and the second errors in the observed flux show statistical and systematic errors, respectively. Expected event rates based on different flux models are also shown. Fluxes $\mathrm{A}, \mathrm{B}$ and $\mathrm{C}$ refer [28], [25] and [29], respectively.

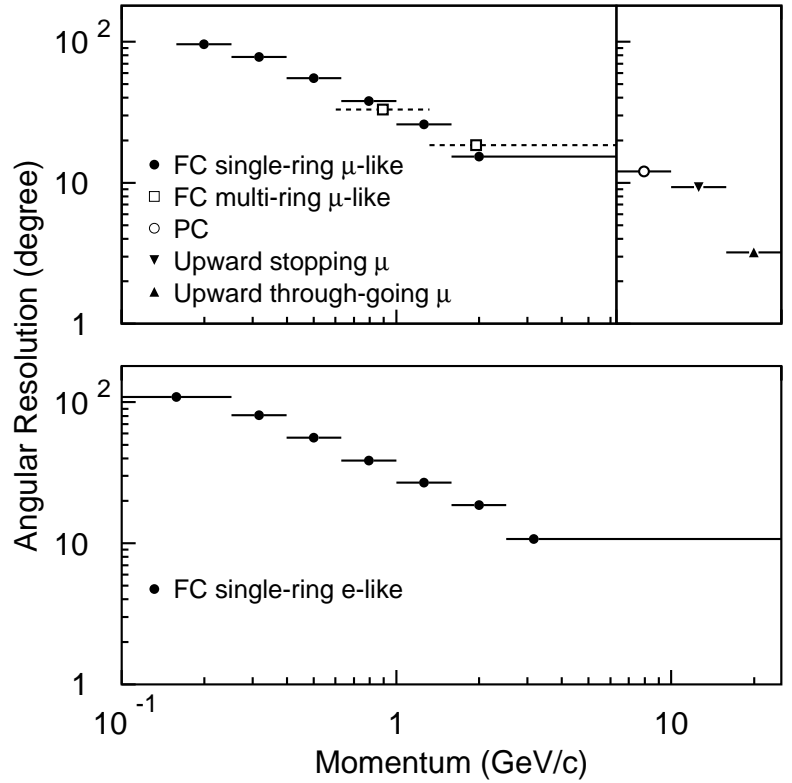

FIG. 32: Angular resolution of the neutrino direction as a function of the outgoing charged lepton momentum. The angular resolution is defined as the angular difference between the parent neutrino and the reconstructed direction for which $68 \%$ of the events are included.

the zenith angle distribution is predicted accurately. Therefore, the vertical to horizontal ratio was taken to study the effects of neutrino oscillations, where $V$ and $H$ represent the number of through-going events with $-1<\cos \Theta<0.5$ and $-0.5<\cos \Theta<0$, respectively. The $V / H$ ratio for the data was $0.497 \pm 0.022$ (stat) \pm 0.003 (sys), while the no-oscillation prediction was $0.586 \pm 0.019$ (sys). Taking into account statistical, systematic, and theoretical uncertainties (using the methods discussed in Section $\mathrm{VI}$, the $V / H$ ratio of the upward through-going muon sample was smaller than the prediction by 3 standard deviations. The observed flux falls off much more rapidly than predicted as the zenith angle approaches the nadir. Fig. 36 shows the expected and observed $\mathrm{V} / \mathrm{H}$ ratio of the upward through-going muon events. The observed ratio suggests that $\Delta \mathrm{m}^{2}$ lies in the range of either $(1-3) \times 10^{-3}$ or $(5-10) \times 10^{-2} \mathrm{eV}^{2}$.

The large uncertainty in the absolute flux normalization can be canceled by taking the stopping to through-going muon ra-
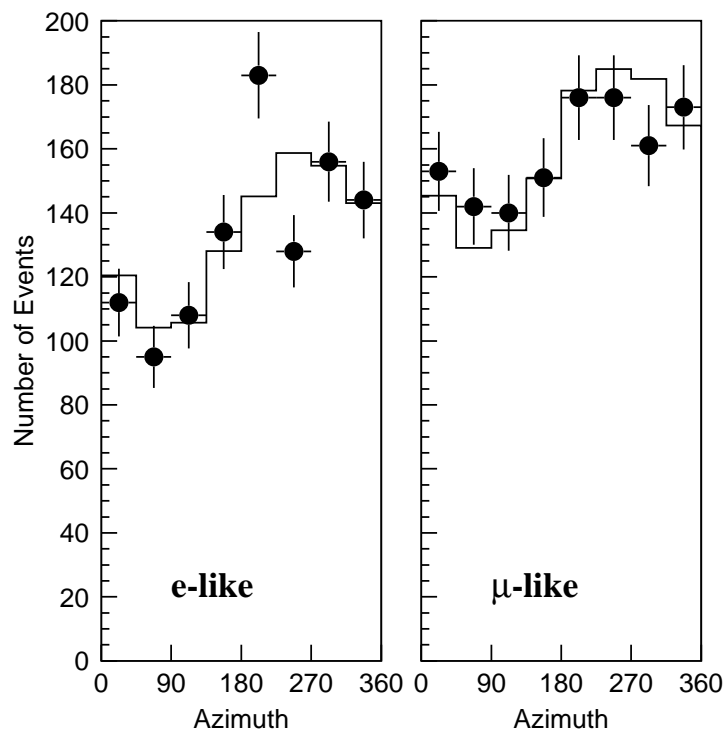

FIG. 33: The azimuth distribution of the sample of events selected for analysis of the east-west effect. The Monte Carlo histogram is normalized to the total number of the real data. $0,90,180$ and 270 degree azimuthal angles show particles going to north, west, south and east, respectively.

tio. Fewer upward stopping muons were observed than predicted, while the observed number of upward through-going muons was consistent with the theoretical prediction within the errors. The observed ratio of stopping to through-going muons was $0.229 \pm 0.015$ (stat) \pm 0.003 (sys), while the expected ratio was $0.430 \pm 0.065$. The expected ratio has theoretical uncertainties from cross sections $( \pm 4.7 \%)$, the cosmic ray spectral index $( \pm 12.5 \%)$, and the flux model dependence $( \pm 7.1 \%)$. Fig. 37 shows the expected ratio of stopping to through-going upward muon events as a function of $\Delta m^{2}$ along with the measured ratio, which was smaller than the prediction by more than 3 standard deviations. The observed value can be explained assuming neutrino oscillations with $\Delta \mathrm{m}^{2}$ in the range of $10^{-3}$ to $10^{-2} \mathrm{eV}^{2}$. This stopping to through-going ratio is no longer explicitly used in the oscillation fits, but is presented for comparison to older work [4]. 

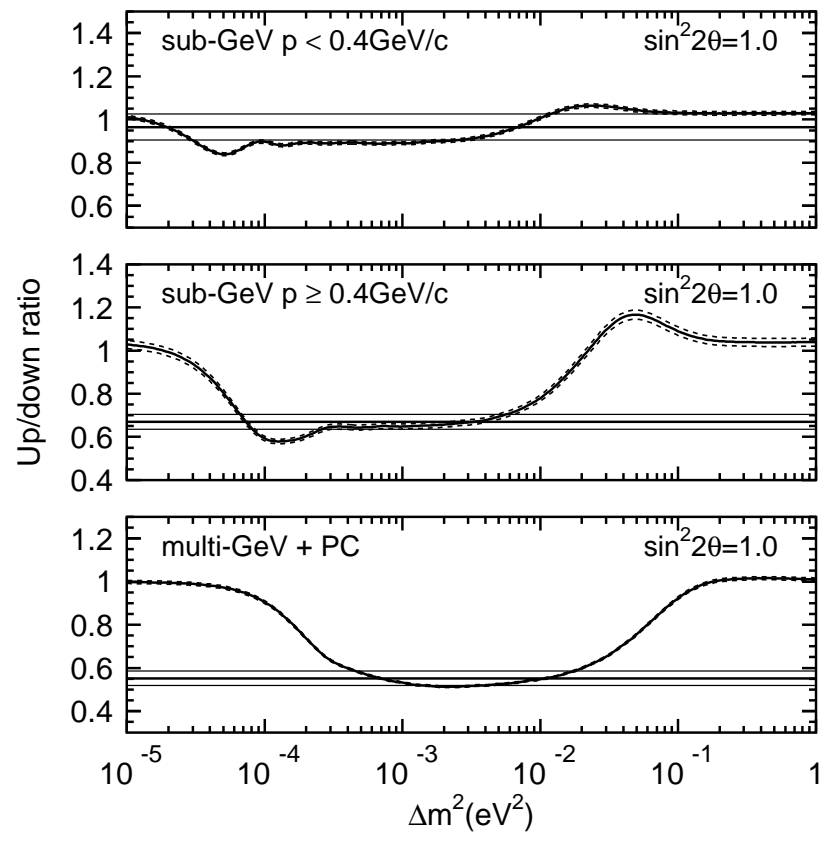

FIG. 34: Expected $U / D$ ratio for FC single-ring $\mu$-like + PC events as a function of $\Delta m^{2}$ for full $\nu_{\mu} \leftrightarrow v_{\tau}$ mixing. The data sample is divided into FC sub-GeV with $P_{\mu}<400 \mathrm{MeV} / c, \mathrm{FC}$ sub-GeV with $P_{\mu}>400 \mathrm{MeV} / c$, and FC multi-GeV + PC events. The ratio for the data together with the $\pm 1 \sigma$ statistical error are shown by the horizontal lines. The systematic errors are shown by the band in the expectation.

\section{OSCILLATION ANALYSIS}

The observed deficits of muon neutrino interactions are in strong disagreement with the expectation in the absence of neutrino oscillations. Oscillation between electron neutrinos and muon neutrinos cannot explain the data, as no surplus of upward-going electron neutrinos is observed in the multi-GeV data sample; an attempt at a two-flavor $v_{\mu} \leftrightarrow v_{e}$ fit results in a generally poor fit, with $\chi^{2}$ difference of more than 100 with respect to the $v_{\mu} \leftrightarrow v_{\tau}$ analysis described below. A variety of exotic alternatives such as neutrino decay were considered, however, none fit the data as well as the $v_{\mu} \leftrightarrow v_{\tau}$ scenario analyzed below. Atmospheric $v_{\mu}$ oscillation into $v_{\tau}$ is mostly characterized by $v_{\mu}$ disappearance, as the majority of the flux is below the $3.5 \mathrm{GeV}$ neutrino energy threshold for charged current $\tau$ production. We carefully studied the alternative that $v_{\mu}$ could oscillate to a sterile neutrino state [81], which would also result in $v_{\mu}$ disappearance. However, the lack of matterinduced suppression of oscillation and the relative up-down symmetry of the multi-ring sample with considerable neutral current fraction eliminated this hypothesis from serious considerations. The final Super-Kamiokande statistical analysis of these alternative scenarios, as well as the standard three flavor oscillation analysis, will appear in other publications. In this paper, we therefore establish the best-fit parameters of $v_{\mu} \leftrightarrow v_{\tau}$ oscillation.

The analysis is based on a comparison between data and Monte Carlo, suitably binned to convey information about

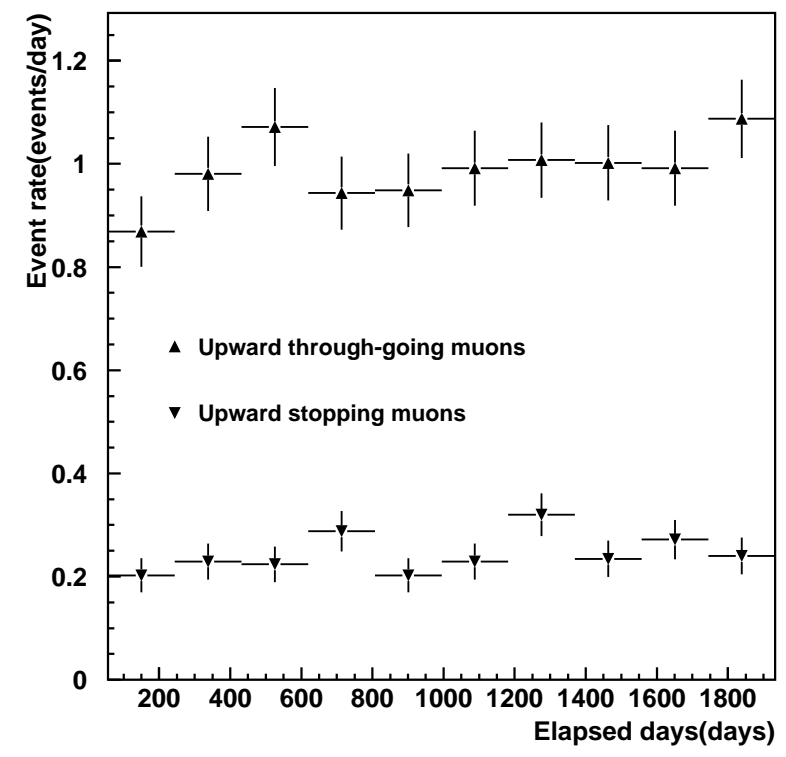

FIG. 35: Event rates as a function of elapsed SK running time for upward-going muon data.

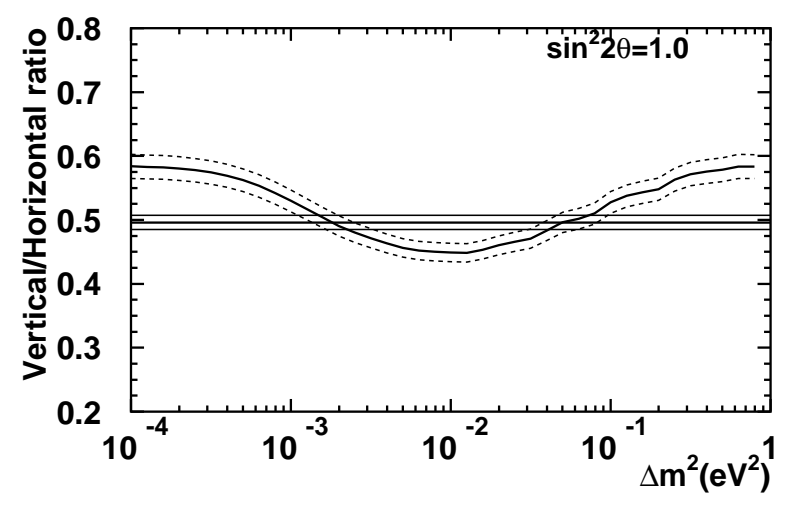

FIG. 36: Expected ratio of vertical to horizontal upward throughgoing muons as a function of $\Delta m^{2}$ for full $v_{\mu} \leftrightarrow v_{\tau}$ mixing. Vertical and horizontal are defined to be the number of events in $-1<$ $\cos \Theta<-0.5$ and $-0.5<\cos \Theta<0.0$, respectively. The ratio for the data together with $\pm 1 \sigma$ combined statistical and systematic error is also shown by the horizontal lines. The systematic error is shown by the band in the expectation.

neutrino type, neutrino energy, and flight distance. The neutrino type, $v_{e}$ or $v_{\mu}$ is classified by the identification of the main Cherenkov pattern as showering or non-showering respectively. Penetrating particles such as upward-going muons and partially-contained events are assumed to arise from $v_{\mu}$ interactions. The neutrino energy is correlated with the outgoing lepton momentum using the interaction models described in Section IV. The flight distance is correlated with the zenith angle as described by Figs. 7 and 32 To study neutrino oscillation using Eq.11 we reweight each simulated event using the Monte Carlo "truth" information of $E_{v}$ and $L$ and bin the reweighted events for comparison with the detected data. Unlike our analysis using the ratio $L / E[86$, we make no attempt to estimate $L$ or $E_{v}$ on an event-by-event basis." 


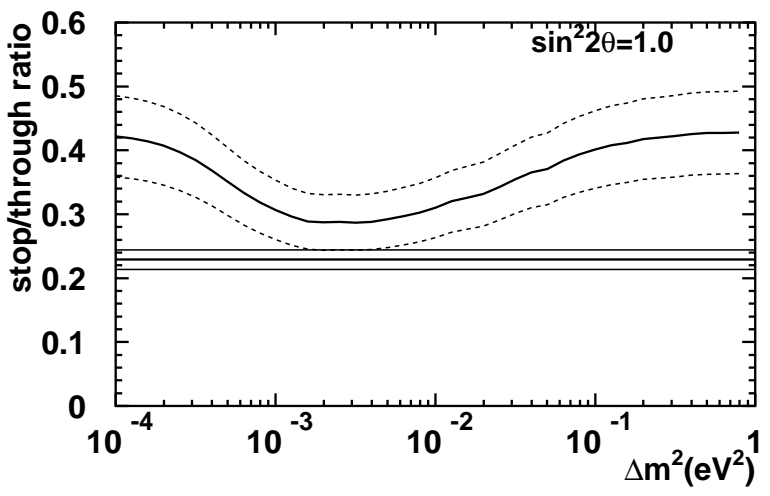

FIG. 37: Expected ratio of stopping to through-going upward muons as a function of $\Delta m^{2}$ for full $v_{\mu} \leftrightarrow v_{\tau}$ mixing. The ratio for the data together with $\pm 1 \sigma$ combined statistical and systematic error is also shown by the horizontal lines. The systematic error is shown by the band in the expectation.

We used all of the data samples with a well-identified CC $v_{\mu}$ component, namely: FC single-ring $\mu$-like, PC, multi-ring $\mu$-like, upward stopping muons, and upward through-going muons. Because the flux of electron neutrinos provides a powerful constraint through the accurately predicted $v_{\mu} / v_{e}$ ratio, the single-ring $e$-like events were included in the fit. The FC single ring $\mu$-like and $e$-like samples were divided in logarithmically-spaced momentum bins. All samples were divided in 10 zenith angle bins. In total 180 bins were used in the analysis: 150 for the FC sample, 10 for the PC sample, 10 for the upward stopping muon sample, and 10 for the upward through-going muon sample. The number of observed and expected events for each bin are summarized in the Appendix.

A $\chi^{2}$ statistic is defined by the following sum:

$$
\begin{gathered}
\chi^{2}=\sum_{i=1}^{180} \frac{\left(N_{i}^{\text {obs }}-N_{i}^{\exp }\left(1+\sum_{j=1}^{39} f_{j}^{i} \cdot \varepsilon_{j}\right)\right)^{2}}{\sigma_{i}^{2}}+\sum_{j=2}^{39}\left(\frac{\varepsilon_{j}}{\sigma_{j}}\right)^{2} \\
N_{i}^{\exp }=N_{i}^{0} \cdot P\left(v_{\alpha} \rightarrow v_{\beta}\right) .
\end{gathered}
$$

In the first sum, $N_{i}^{\text {obs }}$ is the number of observed events in the $i^{\text {th }}$ bin and $N_{i}^{\exp }$ is the expected number of events based on a Monte Carlo simulation and $\sigma_{i}$ combines the statistical uncertainties in the data and Monte Carlo simulation. During the fit, the values of $N_{i}^{\exp }$ are recalculated to account for neutrino oscillations and systematic variations in the predicted rates due to uncertainties in the neutrino flux model, neutrino cross-section model, and detector response. $N_{i}^{0}$ is the number of events predicted from the $\mathrm{MC}$ without neutrino oscillation for the $i^{\text {th }}$ bin. The appearance of $\nu_{\tau}$ as a result of oscillations is taken into account by adding into the Monte Carlo distributions simulated $v_{\tau}$ interactions which pass all cuts. These events show up mainly in the multi-GeV $e$-like sample, but are not easily distinguished on an event-by-event basis. We are undertaking a separate analysis, to be published later, which will study $v_{\tau}$ appearance in the atmospheric neutrino flux.

The systematic uncertainties are represented by 39 parameters $\varepsilon_{j}$. During the fit, these $39 \varepsilon_{j}$ are varied to minimize $\chi^{2}$ for each choice of oscillation parameters $\sin ^{2} 2 \theta$ and $\Delta m^{2}$. Among these, only 38 contribute to the $\chi^{2}$, since the absolute normalization is allowed to be free. The factor $f_{j}^{i}$ represents the fractional change in the predicted event rate in the $i^{\text {th }}$ bin due to a variation of the parameter $\varepsilon_{j}$. The second sum in the $\chi^{2}$ definition collects the contributions from the systematic uncertainties in the expected neutrino rates. The $\varepsilon_{j}$ are listed in Tables $\mathbf{\nabla I I}, \mathbf{D I I}[\mathbf{I X}$ and $[\mathbf{X}$ with their estimated uncertainties and the resulting best-fit values. Entries of the same number are treated as fully correlated although the effect of the uncertainty varies in size depending on its relative importance to the energy bin of certain sub-samples. For example, the source of the up/down uncertainty (No. 8) is due to the uncertainty in the geomagnetic field effect, especially above the SuperKamiokande detector. The uncertainty is large for low energy neutrinos coming from primary cosmic rays below the geomagnetic cutoff, but the effect of the uncertainty is decreased due to the large scattering angle in the neutrino interactions. As a result, events in the middle energy range are the most influenced by this particular systematic uncertainty. Refer to the footnotes in the tables for more detail.

A global scan was made on a $\left(\sin ^{2} 2 \theta, \log \Delta m^{2}\right)$ grid minimizing $\chi^{2}$ at each point with respect to 39 parameters listed in Tables VII VIII IX and X At each grid point, the local minimum of $\chi^{2}$ are derived by assuming a linear dependence of $N_{i}^{\exp }$ on each of the parameters. At the minimum $\chi^{2}$ location, $\partial \chi^{2} / \partial \varepsilon_{j}=0$ for each of the parameters $\varepsilon_{j}$. As a result, the minimization of $\chi^{2}$ in Eqn. 4 is equivalent to solving the following system of $k=1,39$ linear equations [84]:

$$
\begin{gathered}
\sum_{j=1}^{39}\left[\frac{1}{\sigma_{j}^{2}} \delta_{j k}+\sum_{i=1}^{180}\left(\frac{N_{i}^{\exp } \cdot N_{i}^{\exp } \cdot f_{j}^{i} \cdot f_{k}^{i}}{\sigma_{i}^{2}}\right)\right] \cdot \varepsilon_{j} \\
=\sum_{i=1}^{180} \frac{\left(N_{i}^{\text {obs }}-N_{i}^{\exp }\right) \cdot N_{i}^{\exp } \cdot f_{k}^{i}}{\sigma_{i}^{2}}
\end{gathered}
$$

where $\sigma_{j}$ is the estimated uncertainty in the parameter $\varepsilon_{j}$. One of $\sigma_{j}$ corresponds to the absolute normalization uncertainty. In this case, $1 / \sigma_{j}^{2}$ is set to 0 , since the absolute normalization is a free parameter in our analysis.

The minimum $\chi^{2}$ value, $\chi_{\min }^{2}=174.8 / 177 \mathrm{DOF}$, is located at $\left(\sin ^{2} 2 \theta=1.00, \Delta m^{2}=2.1 \times 10^{-3} \mathrm{eV}^{2}\right)$. The number of DOF is found by 180 terms in the $\chi^{2}$ sum plus 38 systamtic constraints in the $\chi^{2}$ sum minus 39 minimized parameters minus the two physics parameters of $\sin ^{2} 2 \theta$ and $\Delta m^{2}$. The overall normalization is not used as a constraint to $\chi^{2}$. The best-fit values of the parameters $\varepsilon_{j}$ obtained at the global minimum are summarized in Tables VII VIII, IX

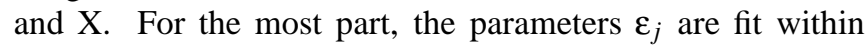
their estimated $1 \sigma$ errors. Including the unphysical region $\left(\sin ^{2} 2 \theta>1\right)$ in the scan, the minimum $\chi^{2}$ value is obtained at $\left(\sin ^{2} 2 \theta=1.02, \Delta m^{2}=2.1 \times 10^{-3} \mathrm{eV}^{2}\right)$. The minimum $\chi^{2}$ value, $\chi_{\text {min }}^{2}=174.5 / 177 \mathrm{DOF}$, in the unphysical region is lower than that in the physical region by 0.29 . Contours corresponding to the $68 \%, 90 \%$ and $99 \%$ confidence intervals are located at $\chi_{\min }^{2}+2.60,4.98$, and 9.60 respectively, where $\chi_{\min }^{2}$ is the minimum $\chi^{2}$ value in the physical region and are shown in Fig. 38 These intervals are derived based on a two dimen- 
$\sigma(\%)$ best-fit $N o$.

\begin{tabular}{|c|c|c|c|c|c|}
\hline \multicolumn{6}{|c|}{ (A) Systematic uncertainties in neutrino flux } \\
\hline \multicolumn{3}{|l|}{ Absolute normalization } & free & 11.9 & 1 \\
\hline \multirow[t]{2}{*}{$\left(\mathrm{v}_{\mu}+\overline{\mathrm{v}}_{\mu}\right) /\left(\mathrm{v}_{e}+\overline{\mathrm{v}}_{e}\right)^{a}$} & $E_{\mathrm{v}}<5 \mathrm{GeV}$ & & 3.0 & -2.4 & 2 \\
\hline & $E_{\mathrm{v}}>5 \mathrm{GeV}$ & & $3.0^{b}$ & 0.1 & 3 \\
\hline \multirow[t]{2}{*}{$\mathrm{v}_{e} / \overline{\mathrm{v}}_{e}^{c}$} & $E_{\mathrm{v}}<10 \mathrm{GeV}$ & & 5.0 & 1.5 & 4 \\
\hline & $E_{\mathrm{v}}>10 \mathrm{GeV}$ & & $5.0^{d}$ & 0.0 & 5 \\
\hline \multirow[t]{2}{*}{$\mathrm{v}_{\mu} / \overline{\mathrm{v}}_{\mu}^{c}$} & $E_{\mathrm{v}}<10 \mathrm{GeV}$ & & 5.0 & -1.3 & 6 \\
\hline & $E_{\mathrm{v}}>10 \mathrm{GeV}$ & & $5.0^{e}$ & 0.9 & 7 \\
\hline \multirow[t]{9}{*}{ Up/down ${ }^{f}$} & $<400 \mathrm{MeV}$ & $e$-like & 0.5 & 0.2 & 8 \\
\hline & & $\mu$-like & 0.8 & 0.3 & 8 \\
\hline & $>400 \mathrm{MeV}$ & $e$-like & 2.1 & 0.9 & 8 \\
\hline & & $\mu$-like & 1.8 & 0.8 & 8 \\
\hline & Multi-GeV & $e$-like & 1.5 & 0.7 & 8 \\
\hline & & $\mu$-like & 0.8 & 0.3 & 8 \\
\hline & $\mathrm{PC}$ & & 0.4 & 0.2 & 8 \\
\hline & Sub-GeV mu & i-ring $\mu$ & 0.8 & 0.3 & 8 \\
\hline & Multi-GeV m & lti-ring $\mu$ & 0.7 & 0.3 & 8 \\
\hline \multirow[t]{9}{*}{ Horizontal/vertical $f^{f}$} & $<400 \mathrm{MeV}$ & $e$-like & 0.3 & 0.0 & 9 \\
\hline & & $\mu$-like & 0.3 & 0.0 & 9 \\
\hline & $>400 \mathrm{MeV}$ & $e$-like & 1.2 & 0.1 & 9 \\
\hline & & $\mu$-like & 1.2 & 0.1 & 9 \\
\hline & Multi-GeV & $e$-like & 2.8 & 0.2 & 9 \\
\hline & & $\mu$-like & 1.9 & 0.1 & 9 \\
\hline & $\mathrm{PC}$ & & 1.4 & 0.1 & 9 \\
\hline & Sub-GeV mu & i-ring $\mu$ & 1.5 & 0.1 & 9 \\
\hline & Multi-GeV m & lti-ring $\mu$ & 1.3 & 0.1 & 9 \\
\hline \multicolumn{3}{|l|}{$K / \pi$ ratio $^{g}$} & 20.0 & -6.3 & 10 \\
\hline \multicolumn{3}{|l|}{$\mathrm{L}_{v}$ (production height) } & $10.0^{h}$ & -0.6 & 11 \\
\hline \multirow[t]{2}{*}{ Energy spectrum $^{i}$} & $E_{k}<100 \mathrm{Ge}$ & & 0.03 & 0.031 & 12 \\
\hline & $E_{k}>100 \mathrm{Ge}$ & & 0.05 & 0.052 & 12 \\
\hline \multirow[t]{2}{*}{ Sample-by-sample ${ }^{j}$} & FC Multi-Ge & & 5.0 & -5.2 & 13 \\
\hline & $\mathrm{PC}+$ upward & topping $\mu$ & 5.0 & -3.9 & 14 \\
\hline
\end{tabular}

${ }^{a} \mathrm{~A}$ positive number means the number of $\mathrm{MC} v_{\mu}+\overline{\mathrm{v}}_{\mu}$ events is increased.

${ }^{b}$ Error linearly increases with $\log E_{v}$ from $3 \%(5 \mathrm{GeV})$ to $10 \%(100 \mathrm{GeV})$.

${ }^{c}$ A positive number means the number of $\mathrm{MC} \mathrm{v}_{e}\left(\mathrm{v}_{\mu}\right)$ events is increased.

${ }^{d}$ Error linearly increases with $\log E_{v}$ from $5 \%(10 \mathrm{GeV})$ to $10 \%(100 \mathrm{GeV})$.

${ }^{e}$ Error linearly increases with $\log E_{v}$ from $5 \%(10 \mathrm{GeV})$ to $25 \%(100 \mathrm{GeV})$.

${ }^{f} \mathrm{Up} /$ down (horizontal/vertical) uncertainty in neutrino flux is assumed to be fully correlated. All of the samples listed are simultaneously varied according to the systematic uncertainty factors. A positive number means the number of MC upward (horizontally-going) events is increased.

${ }^{2} 20 \%$ uncertainty in $K / \pi$ production ratio in cosmic ray interactions in the atmosphere. A positive number means that the fraction of $K$ is increased.

${ }^{h} 10 \%$ uncertainty in the atmospheric density structure. A positive number means a more compressed atmospheric density structure.

${ }^{i} 0.03$ and 0.05 uncertainties in the spectral index of the primary cosmic rays below and above $100 \mathrm{GeV}$. Spectral index uncertainties below and above $100 \mathrm{GeV}$ are assumed to be correlated. A positive number means that the spectrum is harder. The predicted flux was changed around an arbitrary reference energy of $10 \mathrm{GeV}$.

${ }^{j}$ Different flux calculations predict different energy dependences that cannot be explained by a simple spectral index uncertainty. See the lower panel of Fig. 2 From a comparison of the predicted number of events based on different flux models, $5 \%$ is assigned as the relative normalization uncertainty for these samples.

TABLE VII: Summary of systematic uncertainties in the prediction of the atmospheric neutrino flux. Estimated uncertainty and the bestfit value are listed for each error. The last column shows the error parameter numbers $(j)$, which appeared in Eqs 4 and 6 $\overline{\sigma(\%) \text { best-fit } N o .}$

(B) Systematic uncertainties in neutrino interaction

$\begin{array}{llll}M_{A} \text { in quasi-elastic and single- } \pi & 10.0^{a} & 0.5 & 15\end{array}$

$\begin{array}{llll}\text { Quasi-elastic scattering (model dependence) } & 1.0^{b} & -0.95 & 16\end{array}$

$\begin{array}{lllll}\text { Quasi-elastic scattering (cross-section) } & 10.0 & 5.6 & 17\end{array}$

$\begin{array}{llll}\text { Single-meson production (cross-section) } & 10.0 & -4.7 & 18\end{array}$

$\begin{array}{llll}\text { Multi-pion production (model dependence) } & 1.0^{c} & 1.47 & 19\end{array}$

Multi-pion production (total cross-section) $\quad 5.0 \quad-0.2 \quad 20$

$\begin{array}{llll}\text { Coherent pion production (total cross-section) } & 30.0 & 0.4 & 21\end{array}$

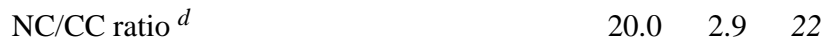

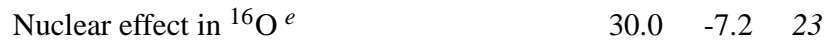

$\begin{array}{llll}\text { Energy spectrum of pions } & 1.0^{f} & 0.50 & 24\end{array}$

\begin{tabular}{llll}
$\mathrm{CC} v_{\tau}$ interaction cross section & 30.0 & 0.2 & 25 \\
\hline
\end{tabular}

${ }^{a} 10 \%$ uncertainty in the axial vector mass, $M_{A}$ (See Sec.IV), value.

${ }^{b}$ Difference from the model in Ref. [82] is set to 1.0 .

${ }^{c}$ Difference from the model in Ref. [83] is set to 1.0 .

${ }^{d} \mathrm{~A}$ positive number means more $\mathrm{NC}$ events in the Monte Carlo.

${ }^{e} 30 \%$ uncertainty in the mean free path of hadrons in the ${ }^{16} \mathrm{O}$ nucleus. A positive number means stronger nuclear effect in ${ }^{16} \mathrm{O}$.

${ }^{f}$ The difference in the predicted pion energy spectrum by NEUT and NUANCE interaction models is taken as 1 standard deviation, and is set to 1.0 .

TABLE VIII: Summary of systematic uncertainties in neutrino interactions. Estimated uncertainty and the best-fit value are listed for each error. The last column shows the error parameter numbers $(j)$, which appeared in Eqs 4 and 6

sional extension of the method described in Ref. [85]. Figure 39] shows the $\chi^{2}-\chi_{\min }^{2}$ distributions projected to $\sin ^{2} 2 \theta$ and $\Delta m^{2}$ axes, in which the minimum $\chi^{2}-\chi_{\text {min }}^{2}$ values for each $\sin ^{2} 2 \theta$ and $\Delta m^{2}$ are plotted. The $\chi^{2}-\chi_{\min }^{2}$ distribution is rather flat between $\Delta m^{2}=2.0 \times 10^{-3} \mathrm{eV}^{2}$ and $2.5 \times 10^{-3} \mathrm{eV}^{2}$. Any $\Delta m^{2}$ in this range fits the data nearly as well as the best-fit point.

Assuming no oscillation, $\left(\sin ^{2} 2 \theta=0, \Delta m^{2}=0\right)$, we found a $\chi^{2}$ value of 478.7 for $179 \mathrm{DOF}$, where only the overall normalization is a free parameter. We allowed all systematic uncertainty terms to be minimized, yet the fit was greatly inferior to the best-fit including neutrino oscillations.

We have also estimated the allowed neutrino oscillation parameters by performing the same fitting procedure using independent subsamples of the data: FC single-ring sub-GeV events below $400 \mathrm{MeV} / c$, FC single-ring sub-GeV events above $400 \mathrm{MeV} / c$, FC single-ring multi-GeV events, PC events, FC multi-ring events, and upward-going muon events. In each independent fit, only the relevant parameters out of the set of 39 were minimized. The results are shown in Fig.40 The allowed region contours found by fitting these six subsamples are consistent with each other and with the combined fit to all events.

In addition, the same oscillation analyses were repeated using different flux models (but with the same neutrino interaction Monte Carlo program) and different neutrino interaction Monte Carlo program (but with the same flux model). The $90 \%$ C.L. allowed parameter regions are compared in Fig.41 


\begin{tabular}{|c|c|c|c|c|c|}
\hline & & & \multicolumn{3}{|c|}{$\sigma(\%)$ best-fit $N o$. } \\
\hline \multicolumn{6}{|c|}{ (C) Systematic uncertainties in event selection } \\
\hline \multicolumn{3}{|c|}{ Reduction for fully-contained event } & 0.2 & 0.0 & 26 \\
\hline \multicolumn{3}{|c|}{ Reduction for partially-contained event } & 2.6 & 0.3 & 27 \\
\hline \multirow[t]{2}{*}{ Detection efficiency $^{a}$} & upward sto & pping $\mu$ & 1.3 & -0.2 & 28 \\
\hline & upward thr & ough-going $\mu$ & 0.5 & -0.1 & 28 \\
\hline \multicolumn{3}{|l|}{ FC/PC separation ${ }^{b}$} & 0.9 & -0.3 & 29 \\
\hline \multicolumn{3}{|l|}{ Hadron simulation } & $1.0^{c}$ & -0.24 & 30 \\
\hline \multirow[t]{5}{*}{ Non- $v \mathrm{BG}^{d}$} & Sub-GeV & $e$-like & 0.4 & 0.1 & 31 \\
\hline & & $\mu$-like & 0.1 & 0.0 & 32 \\
\hline & Multi-GeV & $e$-like & 0.2 & 0.0 & 31 \\
\hline & & $\mu$-like & 0.1 & 0.0 & 32 \\
\hline & $\mathrm{PC}$ & & 0.2 & 0.0 & 32 \\
\hline \multicolumn{3}{|c|}{ Upward stopping/through-going $\mu$ separation ${ }^{e}$} & 0.4 & 0.0 & 33 \\
\hline
\end{tabular}

${ }^{a}$ Goodness of upward-going $\mu$ fit is used to select the upward-going $\mu$ sample. The difference of the goodness between the data and MC is considered as the source of the uncertainty in the detection efficiency. Uncertainties for upward stopping $\mu$ and upward through-going $\mu$ are assumed to be correlated.

${ }^{b}$ The number of hits in the OD cluster is used to separate the FC and PC events. See Fig. 16 The systematic uncertainty in the number of hits in the OD cluster causes $0.9 \%$ uncertainty in the number of the PC events. The number of $\mathrm{FC}$ events changes anti-correlated with the change in the number of PC events. A positive number means that the number of MC FC events is increased.

${ }^{c}$ Difference from the FLUKA model. A positive number means more hadrons, mostly pions, in neutral current interactions are identified as $\mu$-like.

${ }^{d}$ The background sources are flasher PMTs and neutron interactions for $e$ like events and cosmic ray muons for $\mu$-like events. It is assumed that the background sources are un-correlated between $e$-like and $\mu$-like events. The background for sub- and multi-GeV samples in the $e$-like and $\mu$-like events are assumed to be correlated. The background for the PC sample is also assumed to be correlated with the $\mathrm{FC} \mu$-like samples. Only positive numbers are allowed for the background.

${ }^{e}$ The number of hits in the OD cluster at the exit point of a muon is used to separate the upward stopping and through-going muon events. The uncertainty in the number of hits in the OD cluster causes $0.4 \%$ uncertainty in the stopping/through-going ratio. A positive number means that the number of MC stopping muons is increased.

TABLE IX: Summary of systematic uncertainties in event selection. Estimated uncertainty and the best-fit value are listed for each error. The last column shows the error parameter numbers $(j)$, which appeared in Eqs 4 and 6

The allowed regions from these analyses overlap well, demonstrating that the measured parameters do not strongly depend on the choice of flux or interaction model from which we start the fitting procedure. However, the allowed region obtained based on the flux model of Ref. [29] allows for slightly higher $\Delta m^{2}$. We studied the reason for this difference in detail, and found that the main reason was the slightly harder energy spectrum in the upward-going muon energy range (Fig.2].

Finally, we point out that a separate $L / E$ analysis of the same running period [86], using only selected high resolution FC and PC events, gave an allowed oscillation parameter region consistent with this result. This is shown in Fig. 42, with a magnified view of the region and a linear scale in $\Delta m^{2}$. The $L / E$ analysis provided a slightly better constraint in $\Delta m^{2}$ due

\begin{tabular}{|c|c|c|c|c|c|}
\hline \multirow{2}{*}{\multicolumn{4}{|c|}{$\begin{array}{lr}\sigma(\%) \\
\text { (D) Systematic uncertainties in event reconstruction }\end{array}$}} & \multicolumn{2}{|c|}{ best-fit $N o$. } \\
\hline & & & & & \\
\hline \multirow[t]{8}{*}{ Ring separation $^{a}$} & \multirow[t]{2}{*}{$<400 \mathrm{MeV}$} & $e$-like & 6.3 & 2.6 & 34 \\
\hline & & $\mu$-like & 2.4 & 1.0 & 34 \\
\hline & \multirow[t]{2}{*}{$>400 \mathrm{MeV}$} & $e$-like & 3.4 & 1.4 & 34 \\
\hline & & $\mu$-like & 1.3 & 0.5 & 34 \\
\hline & \multirow[t]{2}{*}{ Multi-GeV } & $e$-like & 15.9 & 6.5 & 34 \\
\hline & & $\mu$-like & 6.2 & 2.5 & 34 \\
\hline & \multicolumn{2}{|c|}{ Sub-GeV multi-ring $\mu$} & 3.7 & -1.5 & 34 \\
\hline & \multicolumn{2}{|c|}{ Multi-GeV multi-ring $\mu$} & 7.2 & -2.9 & 34 \\
\hline \multirow[t]{6}{*}{ Particle identification $^{b}$} & \multirow[t]{2}{*}{ Sub-GeV } & $e$-like & 0.6 & 0.2 & 35 \\
\hline & & $\mu$-like & 0.6 & -0.2 & 35 \\
\hline & \multirow[t]{2}{*}{ Multi-GeV } & $e$-like & 0.4 & 0.1 & 35 \\
\hline & & $\mu$-like & 0.4 & -0.1 & 35 \\
\hline & \multicolumn{2}{|c|}{ Sub-GeV multi-ring $\mu$} & 3.4 & -0.9 & 36 \\
\hline & \multicolumn{2}{|c|}{ Multi-GeV multi-ring $\mu$} & 4.7 & -1.2 & 36 \\
\hline \multicolumn{3}{|c|}{ Energy calibration for FC event ${ }^{c}$} & 2.0 & 0.4 & 37 \\
\hline \multicolumn{3}{|c|}{ Energy cut for upward stopping muon } & 1.1 & -0.2 & 38 \\
\hline \multicolumn{3}{|c|}{ Up/down symmetry of energy calibration ${ }^{d}$} & 0.6 & 0.0 & 39 \\
\hline
\end{tabular}

${ }^{a}$ Ring separation uncertainty is assumed to be fully correlated. Namely, if the number of single-ring sub-GeV $e$-like events have to be increased, the number of single-ring multi-GeV $e$-like events and single-ring sub- and multi$\mathrm{GeV} \mu$-like events have to be increased according to the systematic uncertainty factors. On the other hand, in this case, the number of multi-ring $\mu$-like events have to be decreased. A positive number means the number of MC events for the corresponding sample is increased.

${ }^{b}$ The particle identification uncertainty is anti-correlated between $e$-like and $\mu$-like events. It is assumed that the particle identification uncertainty is correlated between sub- and multi-GeV energy regions. However, it is assumed that it is not correlated between single- and multi-ring events. A positive number means the number of MC events for the corresponding sample is increased.

${ }^{c} 2 \%$ uncertainty in the absolute energy scale of the detector. A positive number corresponds to increasing the visible energy of MC events.

${ }^{d} \mathrm{~A}$ positive number means that the energy of $\mathrm{MC}$ events is increased for upward-going direction.

TABLE X: Summary of systematic uncertainties in event reconstruction. Estimated uncertainty and the best-fit value are listed for each error. The last column shows the error parameter numbers $(j)$, which appeared in Eqs 4 and 6

to locating the oscillatory dip; the present analysis constrains $\sin ^{2} 2 \theta$ better due to high statistics in the up-down asymmetry.

\section{CONCLUSION}

Super-Kamiokande has observed more than 15,000 atmospheric neutrino events during the first data taking period between 1996 and 2001. Atmospheric neutrino events observed in Super-Kamiokande have an energy range from about $100 \mathrm{MeV}$ to $10 \mathrm{TeV}$, and a neutrino flight-length from about $10 \mathrm{~km}$ to $13,000 \mathrm{~km}$. These wide energy and flightlength ranges together with high statistics made it possible to study neutrino oscillations. Especially, the predicted up-down 


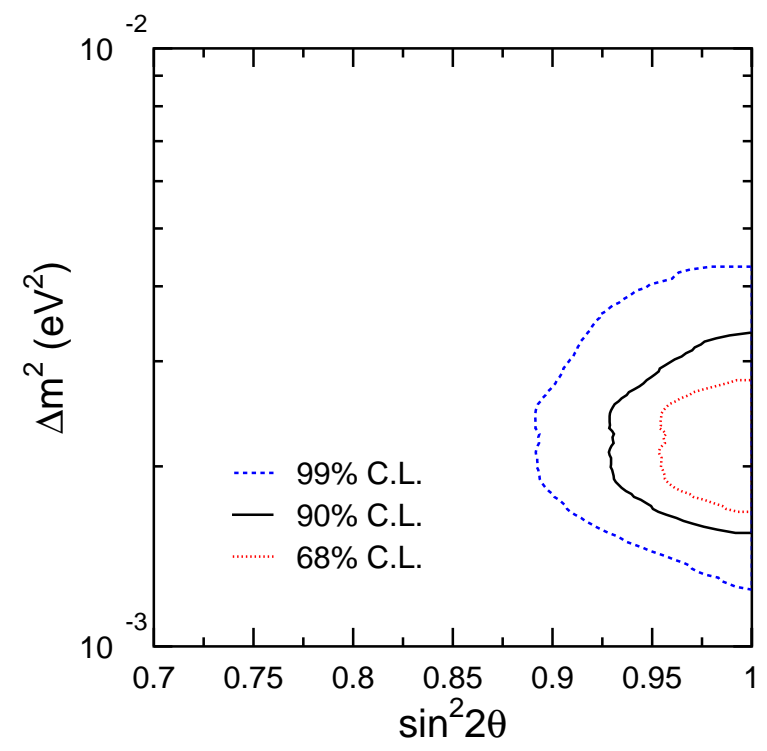

FIG. 38: Allowed oscillation parameters for $v_{\mu} \leftrightarrow v_{\tau}$ oscillations. Three contours correspond to the 68\% (dotted line), $90 \%$ (solid line) and 99\% (dashed line) C.L. allowed regions.
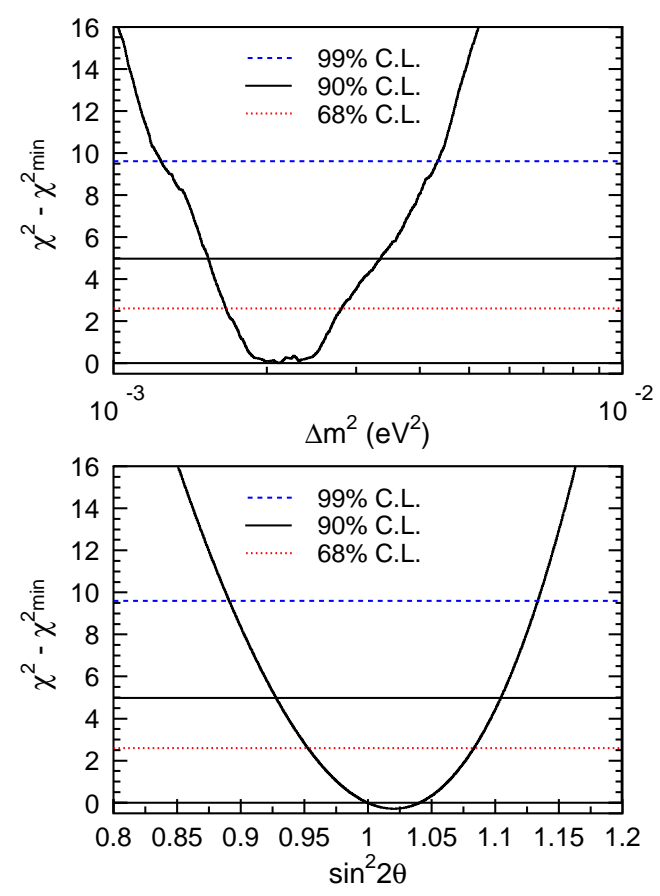

FIG. 39: $\chi^{2}-\chi_{\min }^{2}$ projected onto the $\sin ^{2} 2 \theta$ and $\Delta m^{2}$ axes. The minimum value at each $\sin ^{2} 2 \theta$ and $\Delta m^{2}$ is plotted.

asymmetry of the atmospheric neutrino flux enabled us to accurately estimate the mixing parameter $\sin ^{2} 2 \theta$. The observed muon neutrino events showed a clear zenith angle and energy dependent deficit of events, while the electron neutrino events

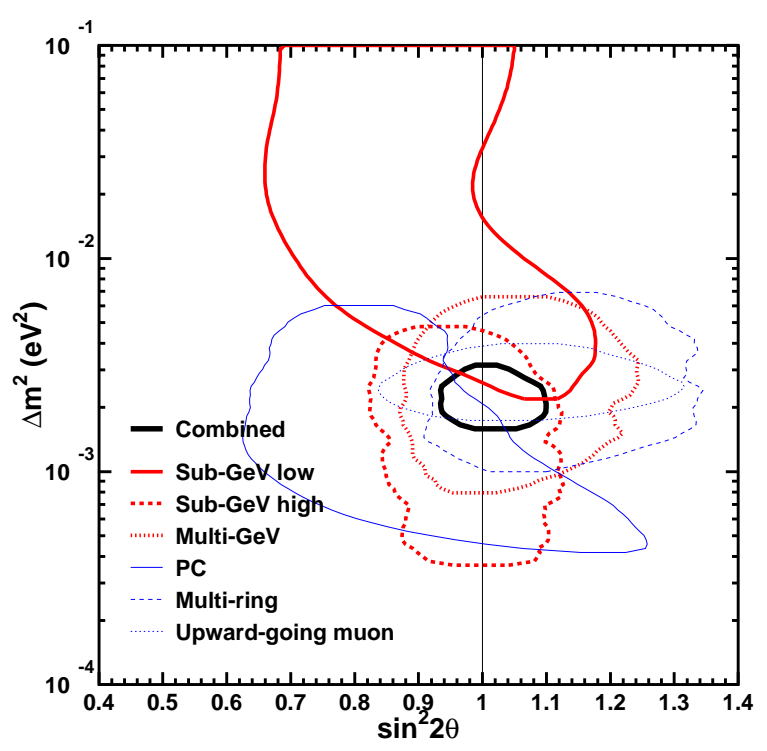

FIG. 40: $90 \%$ confidence level allowed oscillation parameter regions for $v_{\mu} \leftrightarrow v_{\tau}$ oscillations from six sub-samples. In this plot, $90 \%$ confidence interval is defined to be $\chi^{2}=\chi_{\text {min }}^{2}+4.61$, where $\chi_{\text {min }}^{2}$ is the minimum $\chi^{2}$ value including the unphysical parameter region.

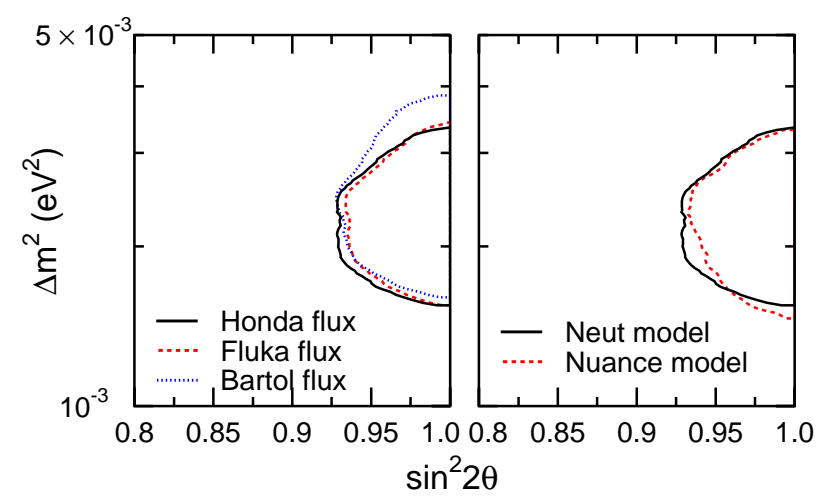

FIG. 41: Left: $90 \%$ confidence level allowed oscillation parameter regions for $v_{\mu} \leftrightarrow v_{\tau}$ oscillations, based on the NEUT neutrino interaction model, from different flux models (solid line; [28], dashed line; [25], dotted line; [29]). Right: The $90 \%$ C.L. allowed regions based on a different neutrino interaction model (NUANCE [48]) for FC+PC events with the flux model of Ref. [28] (dashed line) is compared with that based on NEUT with the same flux. In this plot, Monte Carlo events from NEUT were used for upward-going muons.

were in good agreement with the prediction. A detailed neutrino oscillation analysis confirmed that the full data set was explained well by $v_{\mu} \leftrightarrow v_{\tau}$ oscillations. Various systematic effects were included in the oscillation analysis. The measured neutrino oscillation parameters were $\sin ^{2} 2 \theta>0.92$ and $1.5 \times 10^{-3}<\Delta m^{2}<3.4 \times 10^{-3} \mathrm{eV}^{2}$ at $90 \%$ C.L. This result gives the most accurate determination of $\sin ^{2} 2 \theta$ and is consistent with the somewhat more accurate measurement of $\Delta m^{2}$ 


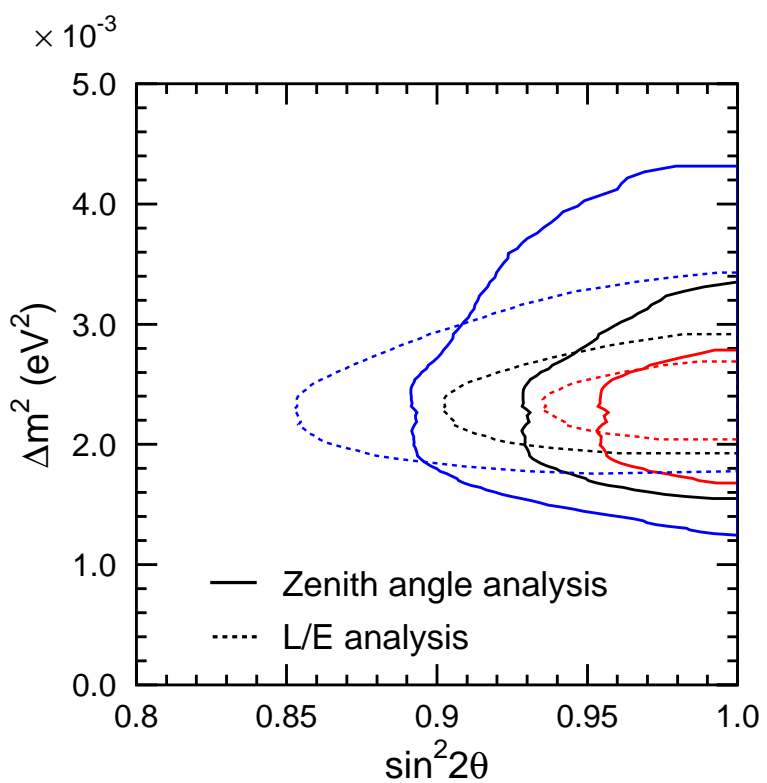

FIG. 42: The 68, 90 and $99 \%$ confidence level allowed oscillation parameter regions obtained by an $L / E$ analysis [86] and by the present analysis are compared. determined by the independent study of high resolution $L / E$ events $[86]$.

We gratefully acknowledge the cooperation of the Kamioka Mining and Smelting Company. The Super-Kamiokande experiment has been built and operated from funding by the Japanese Ministry of Education, Culture, Sports, Science and Technology, the United States Department of Energy, and the U.S. National Science Foundation. Some of us have been supported by funds from the Korean Research Foundation (BK21) and the Korea Science and Engineering Foundation, the State Committee for Scientific Research in Poland (grants 1P03B08227 and 1P03B03826), Japan Society for the Promotion of Science, and Research Corporation's Cottrell College Science Award.

\section{A. Appendix}

Table XI summarizes the number of observed and expected FC and PC events for each bin. The Monte Carlo prediction does not include neutrino oscillations. Table XII summarizes those for upward-going muons. These binned data are used in the oscillation analysis. Table XIII summarizes neutrino energy at which $50 \%$ of events are accumulated for each energy bin in the absence of neutrino oscillations. The fraction of various neutrino interaction modes are also listed.
[1] Y. Fukuda et al. (Super-Kamiokande), Phys. Lett. B433, 9 (1998), hep-ex/9803006.

[2] Y. Fukuda et al. (Super-Kamiokande), Phys. Lett. B436, 33 (1998), hep-ex/9805006.

[3] Y. Fukuda et al. (Super-Kamiokande), Phys. Rev. Lett. 82, 2644 (1999), hep-ex/9812014.

[4] Y. Fukuda et al. (Super-Kamiokande), Phys. Lett. B467, 185 (1999), hep-ex/9908049.

[5] Y. Fukuda et al. (Super-Kamiokande), Phys. Rev. Lett. 81, 1562 (1998), hep-ex/9807003.

[6] K. S. Hirata et al. (KAMIOKANDE-II), Phys. Lett. B205, 416 (1988).

[7] K. S. Hirata et al. (Kamiokande-II), Phys. Lett. B280, 146 (1992).

[8] D. Casper et al., Phys. Rev. Lett. 66, 2561 (1991).

[9] R. Becker-Szendy et al., Phys. Rev. D46, 3720 (1992).

[10] W. W. M. Allison et al., Phys. Lett. B391, 491 (1997), hepex/9611007.

[11] W. W. M. Allison et al. (Soudan-2), Phys. Lett. B449, 137 (1999), hep-ex/9901024.

[12] Y. Fukuda et al. (Kamiokande), Phys. Lett. B335, 237 (1994).

[13] M. Ambrosio et al. (MACRO), Phys. Lett. B434, 451 (1998), hep-ex/9807005.

[14] S. Hatakeyama et al. (Kamiokande), Phys. Rev. Lett. 81, 2016 (1998), hep-ex/9806038.

[15] M. Ambrosio et al. (MACRO), Phys. Lett. B478, 5 (2000), hepex/0001044.

[16] M. Ambrosio et al. (MACRO), Phys. Lett. B566, 35 (2003), hep-ex/0304037.
[17] M. Sanchez et al. (Soudan 2), Phys. Rev. D68, 113004 (2003), hep-ex/0307069.

[18] M. Ambrosio et al. (MACRO), Eur. Phys. J. C36, 323 (2004).

[19] M. H. Ahn et al. (K2K), Phys. Rev. Lett. 90, 041801 (2003), hep-ex/0212007.

[20] E. Aliu et al. (K2K) (2004), hep-ex/0411038.

[21] G. Battistoni et al., Astropart. Phys. 12, 315 (2000), hep$\mathrm{ph} / 9907408$.

[22] M. Honda, T. Kajita, K. Kasahara, and S. Midorikawa, Phys. Rev. D64, 053011 (2001), hep-ph/0103328.

[23] Y. Tserkovnyak, R. Komar, C. Nally, and C. Waltham, Astropart. Phys. 18, 449 (2003).

[24] Y. Liu, L. Derome, and M. Buenerd, Phys. Rev. D67, 073022 (2003), astro-ph/0211632.

[25] G. Battistoni, A. Ferrari, T. Montaruli, and P. R. Sala (2003), (The discontinuity seen in Fig. 3 has been addressed by later calculations. See: http://www.mi.infn.it/ battist/neutrino.html.), hep-ph/0305208.

[26] J. Wentz et al., Phys. Rev. D67, 073020 (2003), hep$\mathrm{ph} / 0301199$.

[27] J. Favier, R. Kossakowski, and J. P. Vialle, Phys. Rev. D68, 093006 (2003), astro-ph/0305460.

[28] M. Honda, T. Kajita, K. Kasahara, and S. Midorikawa, Phys. Rev. D70, 043008 (2004), astro-ph/0404457.

[29] G. D. Barr, T. K. Gaisser, P. Lipari, S. Robbins, and T. Stanev, Phys. Rev. D70, 023006 (2004), (Also, private communication with the authors. A slightly different flux from the one discussed in the paper is used in the present analysis.), astro$\mathrm{ph} / 0403630$. 
FC single-ring e-like

\begin{tabular}{|c|c|c|c|c|c|c|c|c|c|c|}
\hline $\log \left(P_{\text {lep }}\right)$ & $\mathrm{I}$ & II & III & IV & $\mathrm{V}$ & $\mathrm{VI}$ & VII & IIX & IX & $\mathrm{X}$ \\
\hline 1 & $114(79.29)$ & $95(83.33)$ & $74(81.41)$ & $94(82.04)$ & $88(83.99)$ & $91(79.78)$ & $79(79.49)$ & $74(84.19)$ & $91(81.50)$ & $100(82.93)$ \\
\hline 2 & $96(75.62)$ & $93(71.70)$ & $96(73.22)$ & $90(69.44)$ & $89(68.36)$ & $85(68.75)$ & $85(69.49)$ & $74(67.18)$ & $83(71.14)$ & $78(69.68)$ \\
\hline 3 & $76(64.16)$ & $80(66.93)$ & $80(65.78)$ & $69(63.60)$ & $72(64.57)$ & $60(64.06)$ & $69(62.39)$ & $71(61.66)$ & $85(59.72)$ & $63(57.48)$ \\
\hline 4 & $48(45.35)$ & $57(47.92)$ & $62(50.12)$ & $52(50.91)$ & $60(51.62)$ & $74(51.60)$ & $55(50.75)$ & $58(49.13)$ & $60(46.51)$ & $43(42.45)$ \\
\hline 5 & $26(21.68)$ & $35(23.19)$ & $31(25.13)$ & $37(25.84)$ & $24(25.55)$ & $38(25.93)$ & $34(24.96)$ & $24(26.14)$ & $21(23.63)$ & $20(18.51)$ \\
\hline 6 & $33(29.29)$ & $35(33.22)$ & $41(34.92)$ & $37(39.72)$ & $46(42.84)$ & $49(43.86)$ & $49(40.65)$ & $32(39.50)$ & $36(32.03)$ & $36(27.30)$ \\
\hline 7 & $23(21.36)$ & $31(25.66)$ & $28(30.10)$ & $42(37.93)$ & $63(45.70)$ & $37(42.99)$ & $54(35.42)$ & $34(32.54)$ & $22(26.00)$ & $18(19.48)$ \\
\hline \multicolumn{11}{|c|}{ FC single-ring $\mu$-like } \\
\hline 1 & $36(54.73)$ & $40(53.66)$ & $39(54.39)$ & $37(55.09)$ & $35(55.75)$ & $34(53.79)$ & $35(53.46)$ & $45(53.57)$ & $48(52.63)$ & $46(52.10)$ \\
\hline 2 & $86(124.32)$ & $77(122.76)$ & $99(123.28)$ & $86(121.54)$ & $87(119.12)$ & $80(119.67)$ & $91(122.64)$ & $85(117.84)$ & $94(115.67)$ & $76(120.59)$ \\
\hline 3 & $94(118.74)$ & $60(112.36)$ & $81(113.06)$ & $94(115.77)$ & $87(112.80)$ & $84(112.77)$ & $116(113.40)$ & $119(111.91)$ & 97(108.09) & $118(104.54)$ \\
\hline 4 & $52(91.07)$ & 48(87.99) & $53(90.52)$ & $53(91.04)$ & $68(94.68)$ & $68(91.11)$ & $72(89.57)$ & $81(88.15)$ & $91(84.45)$ & $86(82.90)$ \\
\hline 5 & $27(43.35)$ & $22(45.89)$ & $22(44.91)$ & $37(44.48)$ & $25(47.03)$ & $40(47.52)$ & $41(47.91)$ & $41(42.59)$ & $46(44.04)$ & $48(43.60)$ \\
\hline $6+7$ & $34(89.46)$ & $46(86.45)$ & $42(86.48)$ & $49(92.54)$ & $54(96.41)$ & $73(94.68)$ & $95(96.23)$ & $87(88.39)$ & $78(84.46)$ & $93(84.82)$ \\
\hline \multicolumn{11}{|c|}{ FC multi-ring $\mu$-like } \\
\hline sub-GeV & $14(27.57)$ & $8(31.15)$ & $20(33.42)$ & $14(33.68)$ & $25(36.13)$ & $16(35.59)$ & $21(34.12)$ & $32(32.94)$ & $29(28.85)$ & $29(29.12)$ \\
\hline multi-GeV & $18(63.27)$ & $29(63.34)$ & $31(66.89)$ & $28(75.69)$ & $41(86.27)$ & $69(82.70)$ & $55(77.10)$ & $54(73.27)$ & $59(62.44)$ & $55(60.87)$ \\
\hline \multicolumn{11}{|c|}{$\mathrm{PC}$} \\
\hline & $49(88.97)$ & $45(88.81)$ & $59(104.46)$ & $89(125.12)$ & $117(154.61)$ & $156(158.34)$ & $114(128.39)$ & $109(103.00)$ & $85(89.70)$ & $88(88.18)$ \\
\hline
\end{tabular}

TABLE XI: Summary of the number of observed (MC expected) FC and PC events for each bin. Neutrino oscillation is not included in the Monte Carlo prediction. Roman numbers represent zenith angle regions equally spaced between $\cos \Theta=-1$ and $\cos \Theta=1$. The numbers in the $\log \left(P_{l e p}\right)$ column show the momentum ranges. The momentum ranges are $<250,250-400,400-630,630-1000$ and $>1000 \mathrm{MeV} / \mathrm{c}$ in sub-GeV samples for momentum range numbers 1 to 5 and $<2500$ and $>2500 \mathrm{MeV} / \mathrm{c}$ in multi-GeV samples for momentum range numbers 6 to 7 .

upward through-going muon

\begin{tabular}{|r|r|r|r|r|r|r|r|r|r|r|}
\hline & $\mathrm{I}$ & $\mathrm{II}$ & $\mathrm{III}$ & $\mathrm{IV}$ & $\mathrm{V}$ & $\mathrm{VI}$ & $\mathrm{VII}$ & $\mathrm{IIX}$ & $\mathrm{IX}$ & $\mathrm{X}$ \\
\hline \# Events & 85 & 113 & 116 & 138 & 159 & 183 & 178 & 267 & 286 & 316.6 \\
\# Expected & 96.13 & 114.85 & 122.48 & 136.88 & 145.79 & 169.17 & 187.32 & 210.92 & 228.76 & 257.16 \\
Efficiency & $95.2 \%$ & $93.9 \%$ & $92.4 \%$ & $95.9 \%$ & $94.0 \%$ & $97.2 \%$ & $96.9 \%$ & $99.0 \%$ & $96.2 \%$ & $95.5 \%$ \\
\hline Flux & 0.862 & 1.060 & 1.045 & 1.216 & 1.388 & 1.589 & 1.557 & 2.365 & 2.574 & 2.953 \\
Stat. err. & \pm 0.093 & \pm 0.100 & \pm 0.097 & \pm 0.104 & \pm 0.110 & \pm 0.117 & \pm 0.117 & \pm 0.145 & \pm 0.152 & \pm 0.191 \\
Expected & 0.976 & 1.078 & 1.103 & 1.206 & 1.274 & 1.469 & 1.638 & 1.868 & 2.060 & 2.393 \\
Stat. err. & \pm 0.021 & \pm 0.021 & \pm 0.021 & \pm 0.022 & \pm 0.022 & \pm 0.024 & \pm 0.025 & \pm 0.027 & \pm 0.029 & \pm 0.032 \\
\hline
\end{tabular}

upward stopping muon

\begin{tabular}{|r|r|r|r|r|r|r|r|r|r|r|}
\hline \# Events & 28 & 23 & 37 & 30 & 27 & 37 & 37 & 48 & 65 & 85.7 \\
\# Expected & 51.24 & 54.06 & 56.69 & 64.96 & 67.60 & 68.21 & 78.85 & 80.96 & 94.00 & 96.90 \\
Efficiency & $99.8 \%$ & $99.1 \%$ & $99.8 \%$ & $108.1 \%$ & $102.1 \%$ & $101.2 \%$ & $103.5 \%$ & $103.1 \%$ & $105.0 \%$ & $100.2 \%$ \\
\hline Flux & 0.286 & 0.217 & 0.337 & 0.265 & 0.236 & 0.322 & 0.323 & 0.425 & 0.589 & 0.807 \\
Stat. err & \pm 0.054 & \pm 0.045 & \pm 0.055 & \pm 0.048 & \pm 0.045 & \pm 0.053 & \pm 0.053 & \pm 0.061 & \pm 0.073 & \pm 0.172 \\
Expected & 0.523 & 0.509 & 0.517 & 0.574 & 0.591 & 0.594 & 0.689 & 0.715 & 0.851 & 0.913 \\
Stat. err. & \pm 0.015 & \pm 0.015 & \pm 0.015 & \pm 0.015 & \pm 0.015 & \pm 0.015 & \pm 0.016 & \pm 0.017 & \pm 0.019 & \pm 0.020 \\
\hline \hline
\end{tabular}

TABLE XII: Summary of the number of observed and expected upward-going muons for each bin, efficiencies, and the corresponding flux. Neutrino oscillation is not included in the Monte Carlo prediction. The errors on the observed fluxes are statistical, the units of flux $\times 10^{-13} \mathrm{~cm}^{-2} \mathrm{~s}^{-1} \mathrm{sr}^{-1}$. The Roman numerals refer to zenith angle regions equally spaced between $\cos \Theta=-1$ and $\cos \Theta=0$. 
FC single-ring e-like

\begin{tabular}{|c|c|c|c|c|c|c|}
\hline \multicolumn{3}{|c|}{$\log \left(P_{\text {lep }}\right)$} & \multirow{2}{*}{\begin{tabular}{r|}
$E_{\mathrm{V}}(\mathrm{GeV})$ \\
0.31
\end{tabular}} & \multirow{2}{*}{$\begin{array}{r}\mathrm{CC} \mathrm{v}_{e}(\%) \\
87.6\end{array}$} & \multirow{2}{*}{$\begin{array}{r}{\mathrm{CC} v_{\mu}(\%)}_{2.2} \\
\end{array}$} & \multirow{2}{*}{$\begin{array}{r}\mathrm{NC}(\%) \\
10.1\end{array}$} \\
\hline 1 & sub-GeV & $100-250$ & & & & \\
\hline 2 & & $250-400$ & 0.48 & 89.1 & 1.5 & 9.5 \\
\hline 3 & & $400-630$ & 0.72 & 88.7 & 1.8 & 9.5 \\
\hline 4 & & $630-1000$ & 1.1 & 86.8 & 3.2 & 10.1 \\
\hline 5 & & $>1000$ & 1.5 & 86.5 & 4.5 & 8.9 \\
\hline 6 & multi-GeV & $<2500$ & 2.3 & 85.7 & 5.8 & 8.5 \\
\hline 7 & & $>2500$ & 5.4 & 79.0 & 8.4 & 12.6 \\
\hline \multicolumn{7}{|c|}{ FC single-ring $\mu$-like } \\
\hline 1 & sub-GeV & $200-250$ & 0.50 & 0.3 & 90.9 & 8.8 \\
\hline 2 & & $250-400$ & 0.68 & 1.2 & 95.6 & 3.3 \\
\hline 3 & & $400-630$ & 0.86 & 0.7 & 97.7 & 1.6 \\
\hline 4 & & $630-1000$ & 1.2 & 0.5 & 99.0 & 0.4 \\
\hline 5 & & $>1000$ & 1.5 & 0.4 & 99.3 & 0.2 \\
\hline $6+7$ & multi-GeV & & 2.6 & 0.4 & 99.4 & 0.2 \\
\hline \multicolumn{7}{|c|}{ FC multi-ring $\mu$-like } \\
\hline & sub-GeV & & 1.9 & 3.6 & 90.5 & 5.9 \\
\hline & multi-GeV & & 3.6 & 2.3 & 94.9 & 2.7 \\
\hline \multicolumn{7}{|c|}{ PC } \\
\hline & & & \begin{tabular}{l|l}
7.9 \\
\end{tabular} & 1.8 & 97.3 & 0.9 \\
\hline \multicolumn{7}{|c|}{ Upward-going muons } \\
\hline & upward stop & pping muon & 11.1 & 1.0 & 98.6 & 0.4 \\
\hline & upward thro & ough-going muon & 113.5 & 0.2 & 99.7 & 0.1 \\
\hline
\end{tabular}

TABLE XIII: Description of momentum bins used for this analysis, corresponding to raws of Tables XI and XII Also tabulated are the medium parent neutrino energy, and the relative fractions of $\mathrm{CC}_{e}, \mathrm{CC} v_{\mu}$ and NC interactions in the absence of neutrino oscillations as estimated by the Monte Carlo program. 
[30] M. Honda, T. Kajita, K. Kasahara, and S. Midorikawa, Phys. Rev. D52, 4985 (1995), hep-ph/9503439.

[31] V. Agrawal, T. K. Gaisser, P. Lipari, and T. Stanev, Phys. Rev. D53, 1314 (1996), hep-ph/9509423.

[32] Y. Shikaze et al. (2003), proc. of the 28th International Cosmic Ray Conferences (ICRC 2003), Tsukuba, Japan, 31 Jul - 7 Aug 2003, Vol.7, p.4027.

[33] http://ulysses.sr.unh.edu/NeutronMonitor/.

[34] J. Alcaraz et al. (AMS), Phys. Lett. B490, 27 (2000).

[35] T. Sanuki et al., Astrophys. J. 545, 1135 (2000), astro$\mathrm{ph} / 0002481$.

[36] T. K. Gaisser and M. Honda, Ann. Rev. Nucl. Part. Sci. 52, 153 (2002), hep-ph/0203272.

[37] G. Battistoni, A. Ferrari, T. Montaruli, and P. R. Sala, Astropart. Phys. 19, 269 (2003), hep-ph/0207035.

[38] P. Lipari, Nucl. Phys. B (Proc. Suppl.) 81, 159 (2001).

[39] A. Suzuki et al., Nucl. Instrum. Meth. A329, 299 (1993).

[40] T. Tanimori et al., IEEE Trans. Nucl. Sci. 36, 497 (1989).

[41] H. Ikeda et al., Nucl. Instrum. Meth. A320, 310 (1992).

[42] Y. Fukuda et al., Nucl. Instrum. Meth. A501, 418 (2003).

[43] R. Brun and F. Carminati, CERN Programming Library Long Writeup W5013 (1993).

[44] C. Zeitnitz and T. A. Gabriel, Nucl. Instrum. Meth. A349, 106 (1994).

[45] E. Bracci, CERN/HERA 72-1 (1972).

[46] A. S. Carroll et al., Phys. Rev. C14, 635 (1976).

[47] Y. Hayato, Nucl. Phys. Proc. Suppl. 112, 171 (2002).

[48] D. Casper, Nucl. Phys. Proc. Suppl. 112, 161 (2002), hep$\mathrm{ph} / 0208030$.

[49] C. H. Llewellyn Smith, Phys. Rept. 3, 261 (1972).

[50] R. A. Smith and E. J. Moniz, Nucl. Phys. B43, 605 (1972).

[51] D. Rein and L. M. Sehgal, Ann. Phys. 133, 79 (1981).

[52] D. Rein, Z. Phys. C35, 43 (1987).

[53] T. Kitagaki et al., Phys. Rev. D34, 2554 (1986).

[54] S. K. Singh, M. J. Vicente-Vacas, and E. Oset, Phys. Lett. B416, 23 (1998).

[55] F. Cavanna and others (editors) (2004), proceedings, NeutrinoNucleus Interactions in the Few GeV Region (NuInt04), Gran Sasso, March 17-21, 2004.

[56] D. Rein and L. M. Sehgal, Nucl. Phys. B223, 29 (1983).

[57] M. Gluck, E. Reya, and A. Vogt, Z. Phys. C67, 433 (1995).

[58] M. Nakahata et al. (KAMIOKANDE), J. Phys. Soc. Jap. 55,
3786 (1986).

[59] T. Sjostrand, Comput. Phys. Commun. 82, 74 (1994).

[60] S. J. Barish et al., Phys. Rev. D16, 3103 (1977).

[61] S. Bonetti et al., Nuovo Cim. A38, 260 (1977).

[62] S. Ciampolillo et al. (Gargamelle Neutrino Propane), Phys. Lett. B84, 281 (1979).

[63] N. Armenise et al., Nucl. Phys. B152, 365 (1979).

[64] S. V. Belikov et al., Z. Phys. A320, 625 (1985).

[65] G. M. Radecky et al., Phys. Rev. D25, 1161 (1982).

[66] P. S. Auchincloss et al., Z. Phys. C48, 411 (1990).

[67] J. P. Berge et al., Z. Phys. C35, 443 (1987).

[68] V. B. Anikeev et al., Z. Phys. C70, 39 (1996).

[69] A. S. Vovenko et al., Sov. J. Nucl. Phys. 30, 528 (1979).

[70] D. MacFarlane et al., Z. Phys. C26, 1 (1984).

[71] N. Baker et al., Phys. Rev. D25, 617 (1982).

[72] R. Woods and D. Saxon, Phys, Rev. 95, 577 (1954).

[73] L. L. Salcedo, E. Oset, M. J. Vicente-Vacas, and C. GarciaRecio, Nucl. Phys. A484, 557 (1988).

[74] G. Rowe, M. Salomon, and R. H. Landau, Phys. Rev. C18, 584 (1978).

[75] D. Ashery et al., Phys. Rev. C23, 2173 (1981).

[76] E. Davies, Machine Vision: Theory, Algorithms, Practicalities (Academic Press, San Diego, 1997).

[77] S. Kasuga et al., Phys. Lett. B374, 238 (1996).

[78] M. Ambrosio et al. (MACRO), Astropart. Phys. 9, 105 (1998), hep-ex/9807032.

[79] L. V. Volkova, Sov. J. Nucl. Phys. 31, 784 (1980).

[80] T. Futagami et al. (Super-Kamiokande), Phys. Rev. Lett. 82, 5194 (1999), astro-ph/9901139.

[81] S. Fukuda et al. (Super-Kamiokande), Phys. Rev. Lett. 85, 3999 (2000), hep-ex/0009001.

[82] S. K. Singh and E. Oset, Phys. Rev. C48, 1246 (1993).

[83] A. Bodek and U. K. Yang, Nucl. Phys. Proc. Suppl. 112, 70 (2002), hep-ex/0203009.

[84] G. L. Fogli, E. Lisi, A. Marrone, D. Montanino, and A. Palazzo, Phys. Rev. D66, 053010 (2002), hep-ph/0206162.

[85] R. M. Barnett et al. (Particle Data Group), Phys. Rev. D54, 1 (1996).

[86] Y. Ashie et al. (Super-Kamiokande), Phys. Rev. Lett. 93, 101801 (2004), hep-ex/0404034. 